\title{
A radial hydraulic fracture driven by a Herschel-Bulkley fluid
}

\author{
E.A. Kanin ${ }^{a, *}$, E.V. Dontsov ${ }^{b}$, D.I. Garagash ${ }^{c, a}$ and A.A. Osiptsov ${ }^{a}$ \\ ${ }^{a}$ Multiphase Systems Lab, Skolkovo Institute of Science and Technology (Skoltech), 3 Nobel Street, Skolkovo Innovation Center, Moscow 121205, \\ Russian Federation \\ ${ }^{b}$ ResFrac, 555 Bryant Street, \#185 Palo Alto, CA 94301, USA \\ ${ }^{c}$ Department of Civil and Resource Engineering, Dalhousie University, 1360 Barrington Street, Halifax, Nova Scotia B3H 4R2, Canada
}

\section{ARTICLE INFO}

\section{Keywords:}

Hydraulic fracture; Radial (penny-shaped)

model; Herschel-Bulkley fluid; Yield

stress; Power-law rheology; Numeri-

cal methods; Approximate solution.

\begin{abstract}
A B S TR ACT
We analyse the influence of fluid yield stress on propagation of a radial (penny-shaped) hydraulic fracture in a permeable reservoir. In particular, the Herschel-Bulkley rheological model is adopted that includes yield stress and non-linearity of the shear stress. The rock is assumed to be linear elastic, and the fracture is driven by the point source fluid injection with a constant volumetric rate. The fracture propagation condition follows the theory of linear elastic fracture mechanics, and Carter's leak-off law is selected to govern the fluid exchange process between the fracture and formation. The numerical solution for the problem is found using the algorithm based on Gauss-Chebyshev quadrature and Barycentric Lagrange interpolation techniques. We also construct an approximate solution with the help of the global fluid balance equation and the near-tip region asymptote. The latter approximation is computationally efficient, and we estimate its accuracy by comparing the primary crack characteristics such as opening, pressure, and radius with that provided by the full numerical solution. We present examples corresponding to typical field cases and demonstrate that the addition of yield stress can lead to shorter radius and wider opening compared to the corresponding case with a simpler power-law fluid rheology. Further, we quantify the limiting propagation regimes (or vertex solutions) characterised by dominance of a particular physical phenomenon. Relative to the power-law results, there are two new vertices that are associated with domination of yield stress: storage-yield-stress and leak-off-yield-stress. To understand the influence of various problem parameters, we utilise the constructed approximate solution to investigate the dimensionless parametric space for the problem, in which the applicability domains of the limiting solutions are quantified. This enables one to quickly determine whether the yield stress provides a strong influence for a given problem parameters.
\end{abstract}

\section{Introduction}

Hydraulic fractures are fluid-filled tensile cracks propagating in a solid material, such as Earth's crust, and their dynamics is driven by the high-pressure fluid injection. Hydraulic fractures exist in nature in the form of magmafilled dykes (Spence and Turcotte, 1985; Lister, 1990; Rivalta et al., 2015; Dontsov, 2016b) and fluid-filled cracks in glacier beds (Tsai and Rice, 2010; van der Veen, 2007). However, most often they are human-made, and are utilised in the oil and gas fields to enhance production of hydrocarbons (Economides et al., 1989, 2002). Horizontal wells with multistage hydraulic fracturing have quickly become popular in low permeability formations because, in this case, the connection between the reservoir and wellbore has large area (Vishkai and Gates, 2019).

With respect to petroleum applications, hydraulic fracturing fluid is injected through a wellbore into the reservoir rock resulting in the breakdown. A number of parameters can be adjusted to optimise the treatment, such as volumetric injection rate and hydraulic fracturing fluid properties. Besides breaking the rock, the hydraulic fracturing fluid also carries proppant inside the crack channel, which prevents complete crack closure after shut-in. The fracturing fluid rheology and pumping schedule are typically engineered to achieve the following goals (Economides et al., 1989; Barbati et al., 2016): creation of the sufficient crack aperture for proppant placement, minimisation of proppant particle settling (Osiptsov, 2017), reducing the risk of the bridging (Garagash et al., 2019), reduction of fluid leak-off rate into the permeable reservoir, decreasing the energy consumption required to pump fluid (Lecampion and Zia, 2019), and setting the stage for favourable conditions for flowback (Osiptsov et al., 2019).

\footnotetext{
*Corresponding author

e.kanin@skoltech.ru (E.A. Kanin)

ORCID(s): 0000-0003-3065-8244 (E.A. Kanin)
} 
The fracturing fluid is a complex mixture that contains specific additives, e.g., polymeric substances, which allows one to achieve the desired rheological properties (Osiptsov, 2017). Typical fracturing fluid exhibits non-Newtonian shear-thinning behaviour within the specific range of the shear rate values, i.e. the fluid viscosity declines with the shear rate growth, while for small and large shear rates, the fluid viscosity reaches a constant (Moukhtari and Lecampion, 2018). At the same time, the fracturing foams (Gu and Mohanty, 2015; Faroughi et al., 2018; Fu and Liu, 2019), emulsions, suspensions with proppant and fibers (Stickel and Powell, 2005; Osiptsov, 2017) have the shear-thinning rheology with yield stress. The most recent study of the rheology of guar-based cross-linked fluid on high-precision measurement equipment demonstrated Molchanov et al. (2021) that the yield stress is actually an "apparent" yield stress, i.e. the fluid in fact flows at any small shear rate but with a very high viscosity. In other words, there is a plateau in the dependence of the apparent viscosity on the shear rate in the range of small shear rates. Despite this fact, Herschel-Bulkley model is still able to capture the behaviour further away from this plateau.

Bearing in mind that the mixture of hydraulic fracturing fluid with proppant particles is a concentrated suspension, there is a major challenge in modelling suspension transport in a fracture in the entire range of particle volume fractions, varying sometimes from dilute suspension through concentrated flowing suspension (typical of most treatments) to jamming and close packing. A comprehensive fundamental overview of the state of the art in fluid mechanics of suspensions can be found in Morris (2020b), with a more narrow focus on shear-thickening of concentrated suspensions near the close-packing limit given in the review Morris (2020a). Applied issues and challenges in modelling of suspension flow with application to the design of hydraulic fracturing technology can be found in Osiptsov (2017).

Various rheological models are established for describing the fracturing fluid properties. In the case of pure water or slickwater, Newtonian fluid model is often applied for which the shear stress is linearly proportional to the shear rate. When fracturing fluid has shear-thinning behaviour, we can utilise power-law model (Bird et al., 1987). However, it overestimates and underestimates the fluid viscosity at low and high shear rates correspondingly. This discrepancy can be fixed with the help of the more complex rheological models such as Cross fluid (Cross, 1965), Carreau fluid (Carreau, 1972), truncated power-law model (Lavrov, 2015), or Ellis fluid (Brodkey and Brodkey, 1967). The rheological models mentioned above do not take into account yield stress, whose impact on the flow behaviour can be significant for some fracturing fluids. Herschel-Bulkley rheological model (Herschel and Bulkley, 1926) is a generalisation of the powerlaw model, and it includes the yield stress. The limiting cases of Herschel-Bulkley model are Newtonian, power-law and Bingham plastic (Bingham, 1922) models. Despite the Herschel-Bulkley fluid does not capture viscosity saturation for low and high shear rates, it provides important insights to what is happening within the power-law region of the realistic fracturing fluid.

Various numerical models have been developed over time to simulate hydraulic fracture growth, and their geometric complexity increases gradually. The first models have simple two dimensional geometries: Perkins-Kern-Nordgren (PKN) (Perkins et al., 1961; Nordgren et al., 1972), Khristianovich-Zheltov-Geertsma-De Klerk (KGD) (Khristianovic and Zheltov, 1955; Geertsma et al., 1969) and penny-shaped (or radial) (Abe et al., 1976). Recent two decades saw both analytical and numerical development of the KGD and radial models to correctly account for the multiphysical processes and their non-trivial coupling (see review of Detournay (2016) and discussion later in this Section) and provide blueprints and benchmarks for physically sound numerical three dimensional models of more realistic hydraulic fractures: pseudo-3D (Settari et al., 1986; Adachi et al., 2010; Dontsov and Peirce, 2015a) and planar-3D (Lee and Lee, 1990; Vandamme and Curran, 1989; Peirce and Detournay, 2008; Peirce, 2015; Dontsov and Peirce, 2017b). Further, numerical models for simultaneous growth of multiple hydraulic fractures were proposed (Lecampion and Desroches, 2015; Suarez-Rivera et al., 2019; Dontsov et al., 2019a). A more extended overview of hydraulic fracture propagation models and insights about the utilised numerical techniques can be found in the following review papers (Adachi et al., 2007; Detournay, 2016; Lecampion et al., 2018; Nguyen et al., 2020).

Generally, linear elastic fracture mechanics (LEFM) theory is assumed to govern hydraulic fracture propagation in the numerical models, although its limitations have been recently highlighted in Garagash (2019), Liu and Lecampion (2021). According to its basics, the crack advances when the stress intensity factor exceeds the critical value known as the rock toughness (Irvin, 1957). An alternative formulation of this criterion is the square-root behaviour of the crack opening profile near the moving front (known as the toughness asymptote). In the general case, it describes the opening profile along the limited region adjacent to the front (Bunger and Detournay, 2008) because of the realisation of other physical phenomena during propagation besides the brittle rock failure: viscous fluid flow in the crack channel and leak-off to the reservoir. Typical mesh in the numerical hydraulic fracturing simulators is relatively coarse in order to save computation time. That is why the specialised near-tip region models can be preferred over the LEFM asymptote to increase the accuracy of the numerical calculations. The fracture tip model determines the fracture front location for 
each time instance, describes the fracture characteristic profiles (opening, pressure) near the tip, and resolves the impact of all physical processes on the crack propagation (multiscale model). The tip region is investigated via a semi-infinite fracture model, in which the fracture is moving with a constant velocity that corresponds to an instantaneous local velocity of the finite (parent) fracture front. The viscosity and leak-off tip asymptotic solutions for a hydraulic fracture driven by a power-law fluid were obtained by Desroches et al. (1994) and Lenoach (1995), respectively. The effects of the vapour lag presence at the fracture tip are investigated in works (Rubin, 1993; Garagash and Detournay, 2000). The multiscale tip solution incorporating toughness, viscosity and leak-off limiting regimes is investigated in Garagash et al. (2011), and, afterwards, Dontsov and Peirce (2015b) proposed its approximate version which can be easily integrated into planar-3D models, e.g., as it is done in (Dontsov and Peirce, 2017b). In the majority of the models, the fluid exchange process between the fracture and permeable reservoir is governed by Carter's leak-off law. However, the tip model can also be considered with a more complex pressure-dependent formulation as in (Detournay and Garagash, 2003; Kovalyshen, 2010; Kanin et al., 2020d). In the aforementioned near-tip region models, the fluid flow in the fracture channel is assumed to be laminar. The impact of the laminar-to-turbulent flow regime transition inside the fracture channel is analysed by Dontsov (2016c); Lecampion and Zia (2019); Kanin et al. (2020a,c). The generalised asymptotic solutions for a hydraulic fracture driven by a non-Newtonian fluid are developed in the following works: (Dontsov and Kresse, 2018) for the power-law rheology, (Moukhtari and Lecampion, 2018) for the Carreau model and (Bessmertnykh and Dontsov, 2019) for the Herschel-Bulkley model. The influence of cohesive zone and analysis of the LEFM usage constraints in the hydraulic fracture tip problem are examined by Garagash (2019). The near-tip region of a hydraulic fracture with proppant particles is studied in the paper (Bessmertnykh et al., 2020).

The near-tip region models have also been applied for the development of approximate solutions for a finite crack. The idea is the following: the fracture width profile is a continuation of the corresponding characteristic near the moving front. Further, given the width solution, the dynamics of the finite fracture is retrieved from the global fluid balance. This concept is applied for a radial and plane-strain fracture models (Dontsov, 2016a, 2017; Garagash, 2019), as well as for a more general case of an elliptic fracture Dontsov et al. (2019b).

This paper considers a radial hydraulic fracture model in which the crack is formed in a pre-defined plane, and its geometry is axisymmetric relative to the injection point. The numerical model for a penny-shaped fracture in an impermeable rock driven by laminar flow of a Newtonian fluid is investigated by Savitski and Detournay (2002). The toughness dominated regime, i.e. when the fluid viscosity can be neglected, of a radial fracture in a permeable rock is analysed by Bunger et al. (2005) based on the earlier studies for the plane-strain fracture (Garagash, 2000, 2006b). The early-time solution for a plane-stain fracture with a vapour lag is constructed by Garagash (2006c), and, further, extended to the radial fracture geometry by Bunger and Detournay (2007). The numerical model for a radial fracture taking into account the rock toughness, fluid viscosity and leak-off is presented in (Madyarova, 2003) for Newtonian fluid. The limiting cases of a penny-shaped fracture model with three dimensional pore pressure diffusion and poroelastic effects are considered by (Kovalyshen, 2010; Kovalyshen et al., 2013), while the corresponding hydraulic fracture model with the one dimensional fluid exchange is proposed in the paper (Kanin et al., 2020b) where the authors carry out validation of the applicability domain of Carter's leak-off law for the fluid exchange description. The occurrence of turbulent flow regime inside the radial fracture channel is explored by Dontsov and Peirce (2017a); Zolfaghari and Bunger (2019) for pure water and Lecampion and Zia (2019) for slickwater cases. The impact of the power-law fluid rheology on the fracture propagation is firstly studied by Adachi and Detournay (2002); Garagash (2006a) for the plane-strain geometry, and, afterwards, for the penny-shaped fracture in works (Peck et al., 2018a,b), in which the universal fluid velocity based algorithm are utilised (Wrobel and Mishuris, 2015; Perkowska et al., 2016). The same problem is addressed from scaling point of view in (Dontsov, 2019). The power-law dependence of fracture toughness with fracture length is embedded into a radial fracture model by Liu et al. (2019). It is important to highlight that Liu et al. (2019) utilise a numerical algorithms based on Gauss-Chebyshev quadrature and Barycentric Lagrange interpolation techniques discussed earlier by Viesca and Garagash (2018), which allows one to construct an accurate numerical solution of the multi-scale problem without explicit implementation of the near-tip asymptotic solutions. Since the primary focus of the present paper is non-Newtonian fluids, we also should mention the work of Garagash and Sarvaramini (2012) investigating the conditions for the onset of the plain-strain fracture propagation with plastic fluid and the recent papers of Wrobel (2020); Wrobel et al. (2020) where the authors consider PKN and KGD (plane-strain) hydraulic fracture models filled by the fluid with the truncated power-law rheology.

The vast majority of previous studies do not consider the impact of fluid yield stress on propagation of a finite hydraulic fracture, so we would like to fill the gap and discuss this topic on the example of a radial fracture. To take into account the required parameter, we assume that the Herschel-Bulkley model governs the fracturing fluid 


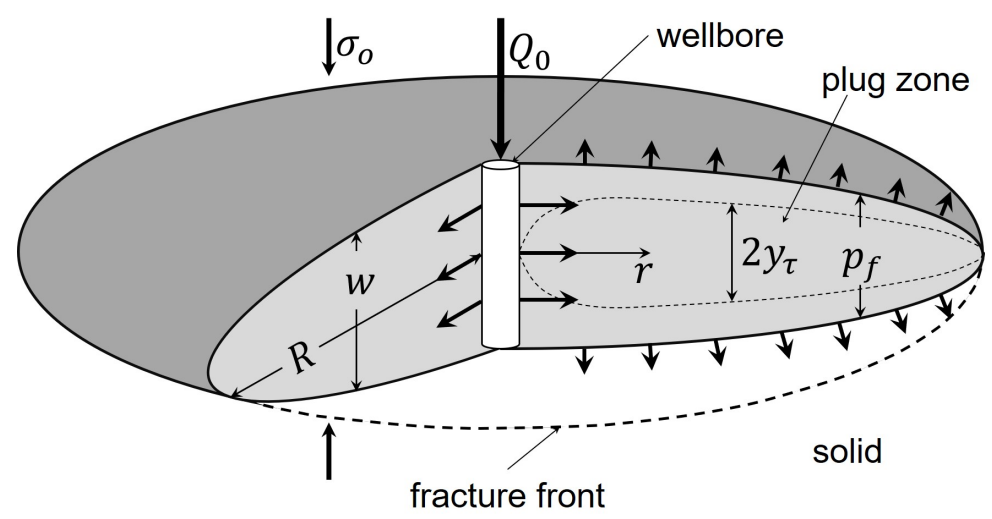

Figure 1: A radial fracture model driven by a Herschel-Bulkley fluid.

rheological behaviour. The main aims of the present work are the following: (i) to implement a solver for calculating an accurate numerical solution for the radial fracture, (ii) to construct an approximate computationally efficient solution for rapid estimations, (iii) to derive limiting propagation regimes occurring in the model, (iv) to explore the problem parametric space and (v) to analyse variations of the fracture characteristics depending on the yield stress and leak-off intensity for different values of the flow index.

The paper is organised in as follows. Section 2 outlines the problem formulation and the governing equations. In Section 3, we describe the methodology for calculating numerical solution, and, after that, we provide insights into getting the simplified approximate solution. Section 4 revisits the known limiting propagation regimes for a radial crack in a permeable rock and introduces the new members associated with dominance of yield stress. In that section, we also establish the conceptual representation of the problem parameter space and discuss the solution trajectories. Section 5 presents analysis of the results, including normalisation of the governing equations, estimation of the admissible ranges for the dimensionless parameters, extensive exploration of the parameter space, as well as discusses examples for several typical field cases. Finally, we summarise the findings of the paper and discuss potential applications of the developed model in Section 6.

\section{Model formulation}

\subsection{Problem definition}

The present paper considers a radial (penny-shaped) hydraulic fracture model whose sketch is shown in Figure 1. The crack grows along the plane perpendicular to the far-field confining stress due to fluid injection through a point source. The fracture is axisymmetric, i.e. there is a symmetry relative to the axis passing through the source and perpendicular to the fracture plane. For the model construction, we introduce the in-plane polar coordinate system $(r, \theta)$ with the origin at the source. Due to symmetry, the spatiotemporal characteristics do not depend on the polar angle $\theta$.

The volumetric rate of fluid injection is constant and is denoted by $Q_{0}$, so that the injected volume is equal to: $V_{\text {inj }}(t)=Q_{0} t$. The ambient rock is taken as linear elastic with Young's modulus $E$ and Poisson's ratio $v$. We assume that the size of the zone, where the dissipation processes associated with the rock failure happen, is small compared to other length scales realised in the model, e.g., linked with the viscous fluid flow and leak-off. Consequently, the linear elastic fracture mechanics (LEFM) theory can be applied to model quasi-static fracture propagation in a solid medium with toughness $K_{I c}$. The fracture surface is exerted by the fluid pressure $p_{f}(r, t)$ from the internal side, while the far-field confining stress is $\sigma_{o}$. The radial fracture model is fully characterised by the opening profile $w(r, t)$, radius $R(t)$, net pressure profile $p(r, t)=p_{f}(r, t)-\sigma_{o}$ and efficiency parameter $\eta(t)=V_{\text {crack }}(t) / V_{\text {inj }}(t)$, where $V_{\text {crack }}(t)$ is the fracture volume.

Hydraulic fracturing fluid has Herschel-Bulkley rheology given by the constitutive relation:

$$
\tau_{m}=\tau_{0}+M \dot{\gamma}^{n},
$$


where $\tau_{m}$ is the shear stress, $\tau_{0}$ is the yield stress, $\dot{\gamma}$ is the shear rate, $M$ is the consistency index, and $n$ is the flow index. When the yield stress is non-zero $\left(\tau_{0}>0\right)$, and the shear stress is less than the yield stress $\left(\tau_{m}<\tau_{0}\right)$, the unyielded (or plug) zone is formed where the fluid behaves like a solid. Since the shear stress is linear across the aperture and equals zero at the centerline, the plug zone is formed in the middle of the crack channel, and its width $2 y_{\tau}(r, t)$ is apriori unknown function of position and time (Figure 1). The Herschel-Bulkley rheological model has three limiting cases: (i) Newtonian fluid when $\tau_{0}=0$ and $n=1$ ( $M$ corresponds to the dynamic viscosity), (ii) power-law fluid when the yield stress is zero $\tau_{0}=0$, and, finally, (iii) it reduces to Bingham model when $\tau_{0} \neq 0$ and $n=1$. The fluid flow inside the fracture channel is controlled by lubrication theory (Batchelor, 1967), its velocity and the flow rate are denoted by $v(r, t)$ and $q(r, t)=w(r, t) v(r, t)$, respectively. The fracture and fluid fronts are assumed to coincide in the model, which means that the lag filled by the vapour (Garagash and Detournay, 2000) or pore fluid (Detournay and Garagash, 2003; Kanin et al., 2020d) adjacent to the fracture front is negligibly small.

The host rock is porous (with porosity $\phi_{r}$ ) and permeable (with permeability $k$ ). The fluid exchange process between the fracture and the formation is taken in the form of the fracturing fluid filtrate leak-off, and its properties are assumed to be similar to water (viscosity $\mu$ and compressibility $c_{t}$ ). The leak-off is modelled by Carter's law (Carter, 1957) which presents the one-dimensional and pressure-independent process. Following this law, the leak-off rate $g(r, t)$ is proportional to the inverse square root of the "exposure time", i.e. the interval between the current time and the time instant at which the fracture tip was at a given point on the fracture plane. The proportionality coefficient is called Carter's leak-off coefficient $C_{L}$. When the filter-cake does not form, and the leaked fluid properties are identical to the pore fluid characteristics (Kovalyshen, 2010; Kanin et al., 2020d), the Carter's coefficient is calculated from: $C_{L}=k\left(\sigma_{o}-p_{o}\right) /(\mu \sqrt{\pi c})$, where $p_{o}$ is the far-field pore pressure, and $c=k /\left(\phi_{r} c_{t} \mu\right)$ is the diffusivity coefficient. The reader can find the expressions for Carter's leak-off coefficient in other cases, e.g., filter-cake presence, in (Economides et al., 1989).

\subsection{Governing equations}

In this section, we discuss the system of governing equations. It is formulated for the unknown crack radius $R(t)$, opening $w(r, t)$ and net fluid pressure $p(r, t)$ profiles. The fracture characteristics depend on time $t$, distance from the point source $r$, the set of material parameters:

$$
E^{\prime}=\frac{E}{1-v^{2}}, \quad K^{\prime}=4 \sqrt{\frac{2}{\pi}} K_{I c}, \quad M^{\prime}=\frac{2^{n+1}(2 n+1)^{n}}{n^{n}} M, \quad C^{\prime}=2 C_{L},
$$

as well as the injection rate $Q_{0}$ and the yield stress $\tau_{0}$. In equation (2), $E^{\prime}$ is the plane strain elastic modulus, $K^{\prime}$ and $M^{\prime}$ are toughness and viscosity parameters, and $C^{\prime}$ is the leak-off parameter.

\subsubsection{Crack elasticity}

The elasticity equation expresses the net fluid pressure $p(r, t)$ in terms of the crack aperture $w(r, t)$ and radius $R(t)$ (Arin and Erdogan, 1971; Cleary and Wong, 1985; Savitski and Detournay, 2002):

$$
p(r, t)=-\frac{E^{\prime}}{2 \pi R(t)} \int_{0}^{R(t)} G\left(\frac{r}{R(t)}, \frac{s}{R(t)}\right) \frac{\partial w(s, t)}{\partial s} d s,
$$

The integral kernel $G(\rho, s)$ in equation (3) has the following form:

$$
G(\rho, s)=\left\{\begin{array}{l}
\frac{1}{\rho} \mathrm{K}\left(\frac{s^{2}}{\rho^{2}}\right)+\frac{\rho}{s^{2}-\rho^{2}} \mathrm{E}\left(\frac{s^{2}}{\rho^{2}}\right), \quad \rho>s, \\
\frac{s}{s^{2}-\rho^{2}} \mathrm{E}\left(\frac{\rho^{2}}{s^{2}}\right), \quad \rho<s,
\end{array}\right.
$$

where $\mathrm{K}(x)$ and $\mathrm{E}(x)$ are the complete elliptic integrals of the first and the second kind, respectively.

\subsubsection{Fluid flow}

Based on lubrication theory (Batchelor, 1967), we write out the width-averaged mass conservation equation for the fluid flow inside the crack channel:

$$
\frac{\partial w}{\partial t}+\frac{1}{r} \frac{\partial(r q)}{\partial r}+g(r, t)=0, q(r, t)=w(r, t) v(r, t) .
$$


The equation for the leak-off rate $g(r, t)$ is provided by Carter's law:

$$
g(r, t)=\frac{C^{\prime}}{\sqrt{t-t_{0}(r)}}
$$

where $t_{0}(r)$ is the inverse fracture radius function: $t_{0}(r)=R^{-1}(t)$, i.e. it indicates the time moment at which the fracture front was at the distance $r$ from the source.

By considering Herschel-Bulkley fluid flowing under a constant pressure gradient inside a constant width channel, one can derive the velocity profile from the combination of the momentum conservation equation and the constitutive relation (1). Averaging the latter across the channel Bessmertnykh and Dontsov (2019), the following equation for the fluid flow velocity is obtained:

$$
v=-\frac{w^{1+1 / n}}{M^{1 / n}} \frac{\partial p}{\partial r}\left|\frac{\partial p}{\partial r}\right|^{1 / n-1}\left(1-\frac{2 y_{\tau}}{w}\right)^{1+1 / n}\left(1+\frac{n}{n+1} \frac{2 y_{\tau}}{w}\right), \quad y_{\tau}=\tau_{0}\left|\frac{\partial p}{\partial r}\right|^{-1},
$$

where $y_{\tau}$ is the half-size of the plug zone. One can notice that at the fluid source and at the tip $y_{\tau}(0, t)=y_{\tau}(R(t), t)=0$ since the pressure gradient is singular there. Therefore, the spatial profile $y_{\tau}(r, t)$ always has an intermediate maximum value as shown schematically in Figure 1.

Equation (7) reduces to Poiseuille's law for Newtonian fluid case when $\tau_{0}=0$ and $n=1$ :

$$
v=-\frac{w^{2}}{M^{\prime}} \frac{\partial p}{\partial r}
$$

and to the power-law fluid case when $\tau_{0}=0$ (Economides et al., 1989):

$$
v=-\frac{w^{1+\frac{1}{n}}}{M^{\prime 1 / n}} \frac{\partial p}{\partial r}\left|\frac{\partial p}{\partial r}\right|^{\frac{1}{n}-1} .
$$

The Reynolds equation is obtained by substitution of the fluid velocity (7) into the continuity equation (5).

\subsubsection{Fracture propagation}

The LEFM theory states that for quasi-static propagation of a hydraulic fracture, the stress intensity factor matches rock toughness: $K_{I}=K_{I c}$. The alternative form to this condition can be expressed in terms of the near-tip asymptotic behaviour of the fracture opening $w(r, t)$ (Irvin, 1957):

$$
w=\frac{K^{\prime}}{E^{\prime}} \sqrt{R-r}, \quad r \rightarrow R
$$

If the crack propagation velocity $V=d R / d t=\dot{R}$ is zero, the tip asymptote has the following form:

$$
w=\frac{K_{I}^{\prime}}{E^{\prime}} \sqrt{R-r}, \quad r \rightarrow R,
$$

where $K_{I}^{\prime}$ is an unknown scaled stress intensity factor, whose value is less than the critical value $K^{\prime}$.

\subsubsection{Boundary conditions}

At the fracture inlet, the volumetric flow rate should be equal to the specified value $Q_{0}$ :

$$
\lim _{r \rightarrow 0} 2 \pi r q(r, t)=\lim _{r \rightarrow 0} 2 \pi r w(r, t) v(r, t)=Q_{o} .
$$

In turn, the crack tip is characterised by zero opening and no-flux condition:

$$
w(R, t)=0, \quad q(R, t)=0 .
$$




\subsubsection{Global fluid volume balance}

By integrating the continuity equation (5) with respect to time and distance and taking into account the boundary conditions (9), (10), we derive the global fluid balance equation:

$$
\underbrace{Q_{0} t}_{V_{\mathrm{inj}}}=\underbrace{2 \pi \int_{0}^{R} r w(r, t) d r}_{V_{\text {crack }}}+\underbrace{4 \pi C^{\prime} \int_{0}^{R} r \sqrt{t-t_{0}(r)} d r}_{V_{\text {leak-off }}} .
$$

It can be interpreted as the injected volume $\left(V_{\text {inj }}\right)$ is distributed between the crack volume $\left(V_{\text {crack }}\right)$ and the volume leaked into the permeable formation $\left(V_{\text {leak-off }}\right)$.

\section{Solution methodology}

This section outlines methods used to compute numerical solution of the posed problem. We utilise two different approaches. The first one is a direct numerical approach that allows us to evaluate the crack parameters accurately, and it is based on Gauss-Chebyshev quadrature and Barycentric Lagrange interpolation techniques. It was first proposed for solving hydraulic fracture propagation problems by Viesca and Garagash (2018). Liu et al. (2019) expanded this methodology for modelling the propagation of finite fractures (radial and KGD), and here, we use their approach and adjust it to capture Herschel-Bulkley rheology and leak-off. Interestingly, due to fine resolution near the tip, the calculation procedure does not require explicit implementation of the full tip asymptotic solution, which differentiates the method from the commonly used approach in which the near tip model is used as a propagation criterion, e.g., (Peirce and Detournay, 2008; Peirce, 2015; Dontsov, 2016a; Dontsov and Peirce, 2017b; Zia and Lecampion, 2020). The second approach helps us to construct the simplified approximate solution based on the full-crack continuation of the near-tip region asymptote and the global fluid balance equation (see (Dontsov, 2016a) for radial crack and (Dontsov, 2017) for KGD crack). The simplified approach is particularly useful to perform rapid estimations of the solution in the whole problem parametric space.

Before moving to the description of algorithms, we introduce the normalised distance from the source: $\rho=r / R(t)$. It depends on time, which results in the following transformation of the derivatives:

$$
\left.\frac{\partial}{\partial t}\right|_{r}=\left.\frac{\partial}{\partial t}\right|_{\rho}-\left.\frac{\rho V(t)}{R(t)} \frac{\partial}{\partial \rho}\right|_{t},\left.\quad \frac{\partial}{\partial r}\right|_{t}=\left.\frac{1}{R(t)} \frac{\partial}{\partial \rho}\right|_{t}
$$

Further, we re-write the elasticity (3), Reynolds (5), (7), and the global fluid balance (11) equations, as well as the propagation condition (8) in terms of $\rho$ :

- Elasticity:

$$
p(\rho, t)=-\frac{E^{\prime}}{2 \pi R(t)} \int_{0}^{1} G(\rho, s) \frac{\partial w(s, t)}{\partial s} d s
$$

For implementation of the numerical solution, we utilise the elasticity equation with an extended interval:

$$
p(\rho, t)=-\frac{E^{\prime}}{4 \pi R(t)} \int_{-1}^{1} G_{e}(\rho, s) \frac{\partial w(s, t)}{\partial s} d s, \quad \rho \in[-1,1],
$$

where the opening profile $w(\rho, t)$ continues symmetrically to negative $\rho$, and the integral kernel has the following form:

$$
G_{e}(\rho, s)=\left\{\begin{array}{l}
\operatorname{sign}(\rho s)\left[\frac{1}{\rho} \mathrm{K}\left(\frac{s^{2}}{\rho^{2}}\right)+\frac{1}{s-\rho} \mathrm{E}\left(\frac{s^{2}}{\rho^{2}}\right)\right],|\rho|>|s|, \\
\frac{1}{s-\rho} \mathrm{E}\left(\frac{\rho^{2}}{s^{2}}\right), \quad|\rho|<|s| .
\end{array}\right.
$$

It can be easily shown that this formulation of the elasticity equation is identical to (3). 
- Reynolds:

$$
\frac{\partial w}{\partial t}-\frac{V}{R} \rho \frac{\partial w}{\partial \rho}+\frac{1}{R \rho} \frac{\partial(\rho w v)}{\partial \rho}+\frac{C^{\prime}}{\sqrt{t-t_{0}(\rho R)}}=0
$$

where the fluid velocity:

$$
v=-\frac{w^{1+1 / n}}{M^{\prime 1 / n} R^{1 / n}} \frac{\partial p}{\partial \rho}\left|\frac{\partial p}{\partial \rho}\right|^{1 / n-1}\left(1-\frac{2 y_{\tau}}{w}\right)^{1+1 / n}\left(1+\frac{n}{n+1} \frac{2 y_{\tau}}{w}\right), \quad y_{\tau}=\tau_{0} R\left|\frac{\partial p}{\partial \rho}\right|^{-1} .
$$

- Global fluid balance:

$$
\frac{Q_{0} t}{\pi R^{2}}=2 \int_{0}^{1} \rho w d \rho+4 C^{\prime} \int_{0}^{1} \rho \sqrt{t-t_{0}(\rho R)} d \rho .
$$

- Propagation condition:

$$
w=\frac{K^{\prime} \sqrt{R}}{E^{\prime}} \sqrt{1-\rho}, \quad \rho \rightarrow 1
$$

\subsection{Numerical solution}

By following the approach of Liu et al. (2019), we present gradient of the fracture opening profile in the form:

$$
\frac{d w}{d \rho}=\mathcal{W}(\rho) F(\rho), \quad \mathcal{W}(\rho)=\frac{1}{\sqrt{1-\rho^{2}}}, \quad \rho \in[-1,1],
$$

where $F(\rho)$ is the required unknown function, and the weight function $\mathcal{W}(\rho)$ includes the tip behaviour of $d w / d \rho$, i.e. $d w / d \rho \sim 1 / \sqrt{1-|\rho|}, \quad \rho \rightarrow \pm 1$. The function $F(\rho)$ is odd, which ensures that the fracture opening profile $w(\rho)$ is symmetric. Further, we perform discretisation of the computational domain $\rho \in[-1,1]$ by introducing two systems of nodes corresponding to the chosen weight function (Viesca and Garagash, 2018): primary $\mathbf{s}=\left\{s_{j}\right\}_{j=1}^{N_{p}}=$ $\left\{\cos \left[\pi(j-1 / 2) / N_{p}\right]\right\}_{j=1}^{N_{p}}$ and complementary $\mathbf{z}=\left\{z_{i}\right\}_{i=1}^{N_{c}}=\left\{\cos \left(\pi i / N_{p}\right)\right\}_{i=1}^{N_{c}}, N_{c}=N_{p}-1$. The bold symbols designate vectors, and $N_{p}$ and $N_{c}$ are the number of primary and complementary nodes, respectively. The primary and complementary nodes are the roots of Chebyshev polynomials of the first $\phi_{N_{p}}(\rho)=\cos \left(N_{p} \theta\right)$ and the second $\psi_{N_{c}}(\rho)=\sin \left[\left(N_{c}+1\right) \theta\right] / \sin (\theta)$ kind, where $\theta=\arccos \rho$. Values of the function $F(\rho)$ are defined at the primary nodes: $\mathbf{F}=\left\{F\left(s_{j}\right)\right\}_{j=1}^{N_{p}}$, while the crack aperture $w(\rho)$ and the net pressure $p(\rho)$ are defined at the complementary nodes: $\mathbf{w}=\left\{w\left(z_{i}\right)\right\}_{i=1}^{N_{c}}, \mathbf{p}=\left\{p\left(z_{i}\right)\right\}_{i=1}^{N_{c}}$.

Now, we move on to the discretisation of the governing equations. For brevity, the matrix notation is utilised. Using the derivations of Liu et al. (2019), we write out the discretised form of the elasticity equation (14):

$$
\mathbf{p}=\frac{E^{\prime}}{4 R} \mathbb{G} \times \mathbf{F}, \quad \mathbb{G}=\mathbb{H}+\frac{1}{2 \mathbf{z}} \mathrm{T} \times \mathbb{H}+\Delta \mathbb{G},
$$

where " $X$ " denotes matrix multiplication; $\mathbb{G}$ is the elasticity quadrature matrix, and its representation composed of the following matrices:

- $\mathbb{H}=\left\{\frac{1}{N_{p}} \frac{1}{z_{i}-s_{j}}\right\}$;

- $\mathbb{T}=\left\{T_{i i^{\prime}}\right\}=\left\{\sum_{k=0}^{N_{c}-1}\left[\Psi_{k}\left(z_{i}\right)-\Psi_{k}(0)\right] B_{k i^{\prime}}\right\}, \Psi_{k}(z)=\cos [(k+1) \theta] /(k+1), \theta=\arccos z$, $B_{k i^{\prime}}=2 / N_{p} \cdot \sin \left(\pi i^{\prime} / N_{p}\right) \sin \left(\pi i^{\prime}(k+1) / N_{p}\right)$

(in this section, symbol $k$ is a summation index, not formation permeability);

- $\Delta \mathbb{G}=\left\{\frac{1}{N_{p}} \Delta G\left(z_{i}, s_{j}\right)\right\}, \Delta G(z, s)=G_{e}(z, s)-\frac{1}{z-s}-\frac{\ln |z-s|}{2 z}$. 
Let us now focus on the discretisation of Reynolds equation (16), (17). Similarly to Liu et al. (2019), we integrate equation (16) with respect to $\rho$ from each node $z_{i}$ to the tip, i.e. $\rho=1$. As a result, we obtain the following discretised form:

$$
\begin{aligned}
& -\frac{\mathbf{z}^{2}}{2} \frac{\partial \mathbf{w}}{\partial t}+\frac{1}{2} \frac{\partial}{\partial t}\left[\mathbb{S} \times\left(\mathbf{s}^{2} \mathbf{F}\right)\right]+\frac{V}{R}\left(\mathbb{S} \times\left(\mathbf{s}^{2} \mathbf{F}\right)\right)-\frac{\mathbf{z w} \mathbf{v}}{R}+C^{\prime} \mathbb{R} \times \frac{\mathbf{z}}{\sqrt{t-t_{0}(\mathbf{z} R)}}=0, \quad \mathbf{w}=\mathbb{S} \times \mathbf{F}, \\
& \mathbf{v}=-\frac{\mathbf{w}^{1+1 / n}}{M^{\prime 1 / n} R^{1 / n}} \mathbf{p}_{\rho}\left|\mathbf{p}_{\rho}\right|^{1 / n-1}\left(1-\frac{2 \mathbf{y}_{\tau}}{\mathbf{w}}\right)^{1+1 / n}\left(1+\frac{n}{n+1} \frac{2 \mathbf{y}_{\tau}}{\mathbf{w}}\right), \quad \mathbf{y}_{\tau}=\frac{\tau_{0} R}{\left|\mathbf{p}_{\rho}\right|}, \quad \mathbf{p}_{\rho}=\frac{E^{\prime}}{4 R} \mathbb{D} \times(\mathbb{G} \times \mathbf{F}),
\end{aligned}
$$

where vector $\left|\mathbf{p}_{\rho}\right|$ contains the absolute values of the pressure gradient at the complementary nodes, and the following matrices are introduced (Liu et al., 2019):

- $\mathbb{S}=\left\{S_{i j}\right\}=\left\{\sum_{k=0}^{N_{c}}\left[\Phi_{k}\left(z_{i}\right)-\Phi_{k}(1)\right] B_{k j}\right\}$, where $\Phi_{k}(z)=-\sin (k \theta) / k, \theta=\arccos z$ and

$B_{k j}=1 / N_{p}$ for $k=0$ and $2 / N_{p} \cdot \cos \left[\pi k(j-1 / 2) / N_{p}\right]$ for $k>0$;

- $\mathbb{R}=\left\{R_{i i^{\prime}}\right\}=\left\{\sum_{k=0}^{N_{c}-1}\left[\Psi_{k}(1)-\Psi_{k}\left(z_{i}\right)\right] B_{k i^{\prime}}\right\}$;

- $\mathrm{D}=\left\{D_{i i^{\prime}}\right\}=\left\{\frac{\omega_{i^{\prime}} / \omega_{i}}{z_{i}-z_{i^{\prime}}}\right.$ for $i \neq i^{\prime} ;-\sum_{i^{\prime}=1, i^{\prime} \neq i}^{m} D_{i i^{\prime}}$ for $\left.i=i^{\prime}\right\}$, where $\omega_{i}=(-1)^{i} \sin ^{2}\left(\pi i / N_{p}\right)$.

To account for the boundary condition at the source, we should embed the global fluid balance into the discretised system. For that purpose, equation (18) is differentiated with respect to time:

$$
\frac{Q_{0}}{\pi}\left(\frac{1}{R^{2}}-\frac{2 t V}{R^{3}}\right)=-S_{H} \times\left(\mathbf{s}^{2} \dot{\mathbf{F}}\right)+2 C^{\prime} \mathbb{R}_{H} \times \frac{\mathbf{z}}{\sqrt{t-t_{0}(\mathbf{z} R)}}-2 C^{\prime} \frac{V}{R^{3}} \int_{0}^{t} \frac{R^{2}(s) d s}{\sqrt{t-s}},
$$

where the matrices $\mathbb{S}_{H}$ and $\mathbb{R}_{H}$ are:

$$
\begin{aligned}
& \text { - } \mathbb{S}_{H}=\left\{\sum_{k=0}^{N_{c}}\left[\Phi_{k}(1)-\Phi_{k}(0)\right] B_{k j}\right\} ; \\
& \text { - } \mathbb{R}_{H}=\left\{\sum_{k=0}^{N_{c}-1}\left[\Psi_{k}(1)-\Psi_{k}(0)\right] B_{k i^{\prime}}\right\} .
\end{aligned}
$$

In equation (22), the last term on the right-hand-side, i.e., the integral with respect to time, is computed using Simpson's rule.

Finally, we write out the propagation condition in the matrix form (see details in (Liu et al., 2019)) and differentiate it with respect to time:

$$
\mathbf{Q} \times \dot{\mathbf{F}}=-\frac{K^{\prime} V}{2 \sqrt{2 R} E^{\prime}}
$$

where $\mathbf{Q}=\left\{Q_{j}\right\}_{j=1}^{N_{p}}=\left\{(-1)^{j+1} \frac{\cot \left(\theta_{j} / 2\right)}{N_{p}}\right\}_{j=1}^{N_{p}}$, where $\theta_{j}=\arccos s_{j}=\pi(j-1 / 2) / N_{p}$.

Here, we should make a comment regarding the application of the propagation condition (23). The LEFM asymptote (19) is always valid near the fracture tip. In certain situations, e.g., vanishingly small toughness, its spatial applicability domain can be tiny. Even when the vanishing LEFM region can not be accurately resolved by the method, Chebyshev's nodes high density near the tip accurately captures the dominant asymptotic behaviour.

Further, we combine equations (21), (22), (23) into the system of ordinary differential equations (ODEs) which can be written as:

$$
\mathbb{A}(\mathbf{X}, t) \frac{d \mathbf{X}}{d t}=\mathbf{B}(\mathbf{X}, t), \quad \mathbf{X}=\{R, \mathbf{F}\}
$$

where the vector $\mathbf{X}$ consists of the unknown parameters, and we use the fact that $V=\dot{R}$. 
The value of $N_{p}$ should be odd since the opposite choice leads to the presence of the infinite components in the elasticity matrix $\mathbb{G}$ (20) (see $\mathbf{z}$ in the denominator). The function $F(\rho)$ is odd, and the the vector $\mathbf{F}$ has in the following form (Liu et al., 2019): $\mathbf{F}=\left\{F_{1}, \ldots F_{\left(N_{p}-1\right) / 2}, 0,-F_{\left(N_{p}-1\right) / 2}, \ldots,-F_{1}\right\}$. The total number of the independent unknown parameters is $\left(N_{p}-1\right) / 2+1$, i.e., radius and independent components of the vector $\mathbf{F}$, meaning that the system of ODEs should be composed of the first $\left(N_{p}-1\right) / 2-1$ discretised Reynolds equations (21), i.e., corresponding to the complementary nodes $z_{1}, \ldots, z_{\left(N_{p}-1\right) / 2-1}$, the global fluid balance equation (22), and the propagation condition (23). We choose $N_{p}=101$, and the storage-viscosity dominated regime ( $M$-vertex) for the power-law fluid is taken as an initial condition for all considered values of the flow index (the fracture properties in this regime are provided by Section 4.1). Despite the fact that for $n<0.5$ the early time solution corresponds to the storage-toughness regime ( $K$ vertex, see details in Section 4.1), the algorithm works fine with the selected initial condition, even though requiring a certain time-span to adjust the solution to the actual trajectory. The specified time interval is discretised uniformly on a logarithmic mesh, and the solver is applied within each time segment. Python programming language is used for implementation of the numerical algorithm, and the system of resulting ODEs is solved via "solve_ivp" function of SciPy library (Virtanen et al., 2020).

\subsection{Rapid approximate solution}

This section outlines a rapid approximate solution for the problem. This approach is based on the idea that the near-tip region behaviour predominantly determines the finite fracture characteristics. As a result, the crack opening profile is presented in the following form (Dontsov, 2016a):

$$
w(\rho, t)=\left(\frac{1+\rho}{2}\right)^{\lambda}(1-\rho)^{\bar{\delta}} w_{a}(R),
$$

where $w_{a}(s)$ is the opening asymptote near the fracture tip stemming from the solution for the semi-infinite geometry, and it is a function of the distance from the front $s$, material parameters (2), yield stress $\tau_{0}$ and time $t$ (through $R(t)$ and $\dot{R}(t)$ ). In equation (25), we also utilise parameters $\lambda$ and $\bar{\delta}$ : the first one originates from comparisons with the accurate solution for limiting cases (will be defined later), and the second one is a slowly varying parameter from the tip asymptotic solution, i.e. $w_{a}(s) \propto s^{\bar{\delta}}$. Since the fracture radius is a power-law function of time in the known limiting propagation regimes (see e.g. a review paper (Detournay, 2016)), we assume that $R(t) \propto t^{\alpha}$, where $\alpha$ is a slowly varying function of time. Consequently, the inverse radius function is expressed in the form: $t_{0}(\rho R)=t \rho^{1 / \alpha}$. By substituting the opening profile (25) and $t_{0}(\rho R)$ into the global fluid balance equation (18), we obtain:

$$
\frac{Q_{0} t}{2 \pi R^{2}}=w_{a}(R) \int_{0}^{1} \rho\left(\frac{1+\rho}{2}\right)^{\lambda}(1-\rho)^{\bar{\delta}} d \rho+2 C^{\prime} \sqrt{t} \int_{0}^{1} \rho \sqrt{1-\rho^{1 / \alpha}} d \rho .
$$

The integrals in the above equation can be estimated using special functions, and the result becomes:

$$
\frac{Q_{0} t}{2 \pi R^{2}}=w_{a}(R) \mathcal{B}(\lambda, \bar{\delta})+2 C^{\prime} \sqrt{t} \alpha B\left(2 \alpha, \frac{3}{2}\right), \quad \mathcal{B}(\lambda, \bar{\delta})=2^{1+\bar{\delta}}\left[B_{0}\left(\frac{1}{2} ; \lambda+2, \bar{\delta}+1\right)-B_{0}(1 / 2 ; \lambda+1, \bar{\delta}+2)\right],
$$

where $B_{0}(x ; a, b)=B(a, b)-B(x ; a, b), B(a, b)$ is the beta function, and $B(x ; a, b)$ is the incomplete beta function.

As can be seen from the above result, one of the building blocks in the approximate solution is the tip asymptote $w_{a}(R)$. We utilise an approximate near-tip region solution developed in Bessmertnykh and Dontsov (2019), which is computed using a combination of the yield stress dominated tip solution $\left(w_{\tau}\right)$ and the one corresponding to the power-law fluid $\left(w_{\mathrm{pl}}\right)$ :

$$
\begin{aligned}
& w_{a}(R)=\left(w_{\mathrm{pl}}^{\zeta}(R)+w_{\tau}^{\zeta}(R)\right)^{1 / \zeta}, \quad w_{\tau}(R)=\sqrt{\frac{8 \pi \tau_{0}}{E^{\prime}}} R, \quad \zeta=-0.3107 n+1.9924, \\
& \sqrt{\frac{R}{\ell}}=\mathcal{H}\left(\frac{w_{\mathrm{pl}} E^{\prime}}{K^{\prime} \sqrt{R}}, \frac{2 C^{\prime} E^{\prime}}{\sqrt{V} K^{\prime}}\right), \quad \ell=\left(\frac{K^{\prime n+2}}{M^{\prime} V^{n} E^{\prime n+1}}\right)^{2 /(2-n)}, \quad V=\dot{R}=\frac{\alpha R}{t},
\end{aligned}
$$

where the function $\mathcal{H}(\tilde{w}, \chi)$ is defined as follows (Dontsov and Kresse, 2018):

$$
\mathcal{H}(\tilde{w}, \chi)=\left(\delta_{1}(\tilde{w}, \chi) \tilde{w}^{(2+n) /(1+\theta)}\left\{\delta_{m}^{1+\theta} \beta_{m}^{2+n}+\delta_{\tilde{m}}^{1+\theta} \beta_{\tilde{m}}^{2+2 n}\left[\left(1+\frac{\chi}{\tilde{w}}\right)^{n}-1\right]\right\}^{-1 /(1+\theta)}\right)^{(1+\theta) /(2-n)} ;
$$




$$
\begin{aligned}
& \delta_{1}(\tilde{w}, \chi)=\frac{\delta_{m} \delta_{\tilde{m}} V_{m} V_{\tilde{m}}\left[\beta_{m}^{(2+n) / n} V_{\tilde{m}}^{(1+\theta) / n}+\frac{\chi}{\tilde{w}} \beta_{\tilde{m}}^{(2+2 n) / n} V_{m}^{(1+\theta) / n}\right]}{\delta_{\tilde{m}} V_{\tilde{m}} \beta_{m}^{(2+n) / n} V_{\tilde{m}}^{(1+\theta) / n}+\delta_{m} V_{m} \frac{\chi}{\tilde{w}} \beta_{\tilde{m}}^{(2+2 n) / n} V_{m}^{(1+\theta) / n}} ; \\
& V_{m}=1-\tilde{w}^{-(2+n) /(1+\theta)}, \quad V_{\tilde{m}}=1-\tilde{w}^{-(2+2 n) /(1+\theta)} ; \\
& \beta_{m}=\left[\frac{2(2+n)^{2}}{n} \tan \left(\frac{\pi n}{2+n}\right)\right]^{1 /(2+n)}, \quad \beta_{\tilde{m}}=\left[\frac{64(1+n)^{2}}{3 n(4+n)} \tan \left(\frac{3 \pi n}{4+4 n}\right)\right]^{1 /(2+2 n)} ; \\
& \delta_{m}=\frac{2-n}{2+n}, \quad \delta_{\tilde{m}}=\frac{2-n}{2+2 n} \\
& \theta=0.0452 n^{2}-0.1780 n+0.1753 .
\end{aligned}
$$

The parameter $\bar{\delta}$ in the global fluid balance equation (27) can be computed from the following relation:

$$
\bar{\delta}=\frac{\delta+1}{2}, \quad \delta=\frac{w_{\mathrm{pl}}^{\zeta} \delta_{1}+w_{\tau}^{\zeta}}{w_{\mathrm{pl}}^{\zeta}+w_{\tau}^{\zeta}},
$$

where the function $\delta_{1}$ is defined in the equation (29), and it enters approximation for the power-law solution: $w_{\mathrm{pl}}(s) \propto$ $s^{\left(\delta_{1}+1\right) / 2}$, where $s$ is the distance from the tip.

Further, we solve the non-linear algebraic system of equations composed of the global fluid balance (27) and the tip asymptote (28). Let us discuss the overall approach in more details. Initially, the desired time interval is discretised uniformly on a logarithmic scale, and the target parameters $\mathcal{X}=\left\{\alpha, R, w_{a}\right\}$ are computed iteratively step-by-step for each time instant. Let us consider the example of time step $t_{i}$. From the previous result at $t_{i-1}$, we know the values of $\mathcal{X}_{i-1}=\left\{\alpha_{i-1}, R_{i-1},\left(w_{a}\right)_{i-1}\right\}$, and they are used as an initial guess for the current computation. We calculate the solution for two time instants: $t_{i}$ and $t_{i}^{*}$, where the latter is a fraction of the current time moment, e.g., it can be $t_{i}^{*}=0.9 \cdot t_{i}$. Further, the value of $\alpha_{i}$ initially taken as $\alpha_{i-1}$ is updated by using the equation: $\alpha_{i}=d \log (R) / d \log (t)=$ $\left[\log \left(R_{i}\right)-\log \left(R_{i}^{*}\right)\right] /\left[\log \left(t_{i}\right)-\log \left(t_{i}^{*}\right)\right]$. After that, the system is solved again with the new initial guess presented by the parameters from this iteration. The process continues until the convergence in the value of $\alpha_{i}$ is reached. It is also necessary to mention that for the first time step $(i=1)$, we take the initial guess $\alpha_{0}=(2 n+2) /(3 n+6), R_{0}$ and $\left(w_{a}\right)_{0}$ corresponding to the storage-viscosity dominated limiting propagation regime (Section 4.1).

Once the solution $\mathcal{X}$ is computed, the fracture opening profile $w(\rho, t)$ is evaluated using equation (25), while the pressure $p(\rho, t)$ profile is calculated based on the transformed elasticity (13) equation (Dontsov, 2016a):

$$
p=\frac{E^{\prime} w_{a}(R)}{R} \mathcal{F}(\rho, \lambda, \bar{\delta}), \quad \mathcal{F}(\rho, \lambda, \bar{\delta})=\frac{1}{2^{\lambda+1} \pi} \int_{0}^{1} \frac{\partial G(\rho, s)}{\partial s}(1+s)^{\lambda}(1-s)^{\bar{\delta}} d s,
$$

where the function $\mathcal{F}$ is evaluated numerically. Finally, we also compute the fracture efficiency as:

$$
\eta=\frac{2 \pi R^{2} w_{a}(R) \mathcal{B}}{Q_{0} t},
$$

where the function $\mathcal{B}$ is introduced in equation (27).

For brevity of presentation, the exact form of the $\lambda$ function is given in Appendix A. The calculation procedure for $\lambda$ also requires the results of Section 4.1, in which the limiting propagation regimes are outlined for the present model.

\section{Conceptual representation of the solution}

\subsection{Limiting propagation regimes}

Two different physical mechanisms govern the propagation regime of a finite hydraulic fracture (see a review paper Detournay (2016) and references therein). The first one is related to the distribution of the total dissipated energy between the creation of new fracture surface and viscous fluid flow including the movement of the solid plug inside the fracture channel. The latter energy component, namely movement of the solid plug, is included since the fracturing fluid has non-zero yield stress. In this case, the whole fracture can be filled with the un-yielded solid material. The second mechanism is the partitioning of the injected fluid volume between the fracture and the host permeable rock 
(due to leak-off). When the fracture grows, the partitioning of the dissipated energy and the injected volume change over time, leading to the emergence of the various limiting propagation regimes with one dissipation (out of two) and one storage (out of two) mechanisms at different time moments. The leak-off parameter $C^{\prime}$ influences distribution of the injected fluid volume, while the viscosity $M^{\prime}$, toughness $K^{\prime}$, and the yield stress $\tau_{0}$ have an effect on the partitioning of the dissipated energy.

Six limiting regimes (also known as the vertex solutions or vertices) can be distinguished in the current radial fracture model. Four of them correspond to the propagation of a penny-shaped crack filled by a fluid with the powerlaw rheology $\left(\tau_{0}=0\right)$ :

- $\mathbf{M}$ - storage-viscosity $-K^{\prime}=C^{\prime}=\tau_{0}=0$;

- $\tilde{\mathbf{M}}$ - storage-toughness $-K^{\prime}=\tau_{0}=0, C^{\prime} \rightarrow+\infty$;

- $\mathbf{K}$ - leak-off-viscosity $-M^{\prime}=C^{\prime}=\tau_{0}=0$;

- $\tilde{\mathbf{K}}$ - leak-off-toughness $-M^{\prime}=\tau_{0}=0, C^{\prime} \rightarrow+\infty$.

The remaining two regimes correspond to the dominance of the yield stress:

- $\mathbf{T}$ - storage-yield-stress $-K^{\prime}=C^{\prime}=M^{\prime}=0, \tau_{0}>0$;

- $\tilde{\mathbf{T}}$ - leak-off-yield-stress $-K^{\prime}=M^{\prime}=0, \tau_{0}>0, C^{\prime} \rightarrow+\infty$.

Let's now consider scalings associated with the limiting propagation regimes. First of all, we present the main crack characteristics as:

$$
w(r, t)=\epsilon(t) L(t) \Omega\left(\rho, \mathcal{P}_{1}, \mathcal{P}_{2}, \mathcal{P}_{3}\right), \quad p(r, t)=\epsilon(t) E^{\prime} \Pi\left(\rho, \mathcal{P}_{1}, \mathcal{P}_{2}, \mathcal{P}_{3}\right), \quad R(t)=L(t) \gamma\left(\mathcal{P}_{1}, \mathcal{P}_{2}, \mathcal{P}_{3}\right),
$$

where $\rho=r / R(t) ; \mathcal{P}_{1}, \mathcal{P}_{2}, \mathcal{P}_{3}$ are dimensionless evolution parameters depending on $t$, material parameters (2), $Q_{0}$ and $\tau_{0} ; L(t)$ is the length scale (the same order as the crack radius) and $\epsilon(t)$ is a small dimensionless parameter with the meaning of a characteristic strain in the rock.

Further, we substitute the expressions from equation (32) into the system of governing equations written in terms of the normalised distance from the source $\rho$, i.e. the elasticity (13), Reynolds (16), (17), global fluid balance (18) equations and the propagation condition (19):

- Elasticity:

$$
\Pi=-\frac{1}{2 \pi \gamma} \int_{0}^{1} G(\rho, s) \frac{\partial \Omega}{\partial s} d s ;
$$

- Reynolds:

$$
\begin{aligned}
& \mathcal{G}_{v}\left(\left[\frac{\dot{\epsilon} t}{\epsilon}+\frac{\dot{L} t}{L}\right] \Omega+\dot{\Omega} t-\rho\left(\frac{\dot{L} t}{L}+\frac{\dot{\gamma} t}{\gamma}\right) \frac{\partial \Omega}{\partial \rho}\right)+\frac{\mathcal{G}_{c}}{\sqrt{1-\theta_{0}}}= \\
& =\frac{1}{\mathcal{G}_{m}} \frac{1}{\gamma \rho} \frac{\partial}{\partial \rho}\left[\frac{\rho}{\gamma^{1 / n}} \Omega^{2+1 / n} \frac{\partial \Pi}{\partial \rho}\left|\frac{\partial \Pi}{\partial \rho}\right|^{1 / n-1}\left(1-\mathcal{G}_{t} \frac{2 \gamma}{\Omega}\left|\frac{\partial \Pi}{\partial \rho}\right|^{-1}\right)^{1+1 / n}\left(1+\mathcal{G}_{t} \frac{2 \gamma}{\Omega}\left|\frac{\partial \Pi}{\partial \rho}\right|^{-1} \frac{n}{n+1}\right)\right] ;
\end{aligned}
$$

- Global fluid balance:

$$
\frac{1}{\pi \gamma^{2}}=2 \mathcal{G}_{v} \int_{0}^{1} \Omega \rho d \rho+4 \mathcal{G}_{c} \int_{0}^{1} \sqrt{1-\theta_{0}} \rho d \rho
$$

- Propagation condition:

$$
\Omega=\mathcal{G}_{k} \sqrt{\gamma} \sqrt{1-\rho}, \quad \rho \rightarrow 1 .
$$


Here, we utilise the function $\theta_{0}(\rho, t)=t_{0}(r) / t$ and introduce five dimensionless numbers:

$$
\mathcal{G}_{v}=\frac{\epsilon L^{3}}{Q_{0} t}, \quad \mathcal{G}_{c}=\frac{C^{\prime} L^{2}}{Q_{0} \sqrt{t}}, \quad \mathcal{G}_{m}=\frac{Q_{0} M^{\prime 1 / n}}{L^{3} E^{\prime 1 / n} \epsilon^{2+2 / n}}, \quad \mathcal{G}_{t}=\frac{\tau_{0}}{\epsilon^{2} E^{\prime}}, \quad \mathcal{G}_{k}=\frac{K^{\prime}}{E^{\prime} \epsilon \sqrt{L}} .
$$

$\mathcal{G}_{v}$ and $\mathcal{G}_{c}$ parameters quantify the fluid volume stored in the fracture and the volume leaked into the ambient permeable rock, respectively. In turn, the numbers $\left(\mathcal{G}_{m}, \mathcal{G}_{t}\right)$ and $\mathcal{G}_{k}$ are related to the energy dissipation in the fluid flow inside the crack channel in overcoming fluid viscosity and solid plug yield strength and in the brittle rock failure, correspondingly.

To derive various scalings, i.e., $L(t)$ and $\epsilon(t)$ in (32), we set one out of two fluid storage parameters $\left(\mathcal{G}_{v}, \mathcal{G}_{c}\right)$ be equal to one. Similarly, among the three parameters responsible for energy dissipation $\left(\mathcal{G}_{m}, \mathcal{G}_{t}, \mathcal{G}_{k}\right)$, one should be made equal to one. The remaining three dimensionless groups are the evolution parameters $\mathcal{P}_{1}, \mathcal{P}_{2}, \mathcal{P}_{3}$ mentioned earlier. When the evolution parameters are approximately zero in a given scaling, the corresponding limiting propagation regime is realised. From the scaling analysis, we can determine only the dimensional multipliers for the radial crack characteristics, $R, w, p$, in a given 'i-th' scaling. In order to approximately quantify the opening and pressure profiles, we rely on the approximations of Section 3.2:

$$
R_{i}(t)=R_{i}^{*} L_{i}, \quad w_{i}(\rho, t)=\underbrace{w_{i}^{*} \epsilon_{i} L_{i}}_{\left(w_{a}\right)_{i}(R)}\left(\frac{1+\rho}{2}\right)^{\lambda_{i}}(1-\rho)^{\bar{\delta}_{i}}, \quad p_{i}(\rho, t)=\frac{w_{i}^{*}}{R_{i}^{*}} E^{\prime} \epsilon_{i} \mathcal{F}\left(\rho, \lambda_{i}, \bar{\delta}_{i}\right),
$$

where the subscript $i$ indicates the particular limiting regime, and the function $\mathcal{F}$ is defined in equation (30). In order to express the prefactors values $R_{i}^{*}, w_{i}^{*}$ through $\lambda_{i}$, we substitute $R_{i}(t)$ and $\left(w_{a}\right)_{i}(R)$ into the global fluid balance equation (27) (accounting the conditions inherent to the analysed regime) and the appropriate near-tip region asymptote for the crack opening. Further, the parameter $\lambda_{i}$ can be found with the help of the accurate numerical solution (Section 3.1).

Before discussing different scalings and limiting propagation regimes, we introduce the relative errors in the estimation of the radius and opening characteristics provided by the simplified approach (34) compared to the fully numerical solution:

$$
\begin{aligned}
& \Delta\left(R_{i}\right)=\left|\bar{R}_{i}(t)-R_{i}(t)\right| / \bar{R}_{i}(t), \\
& \Delta\left(w_{i}^{0}\right)=\left|\bar{w}_{i}(0, t)-w_{i}(0, t)\right| / \bar{w}_{i}(0, t), \\
& \Delta\left(w_{i}^{\rho}\right)=\max _{\rho}\left[\left|\bar{w}_{i}(\rho, t)-w_{i}(\rho, t)\right| / \bar{w}_{i}(0, t)\right],
\end{aligned}
$$

where $\Delta\left(R_{i}\right)$ is the radius error, $\Delta\left(w_{i}^{0}\right)$ is the error of width at the inlet, $\Delta\left(w_{i}^{\rho}\right)$ is the maximum relative width error. The subscript $i$ denotes the considered limiting regime, and the bar symbol means the fully numerical solution.

We begin with the storage-viscosity scaling and the $M$-vertex solution (subscript " $m$ "). We set $\mathcal{G}_{v}=\mathcal{G}_{m}=1$ and obtain the following formulas for the length scale and the small parameter:

$$
L_{m}=\left(\frac{E^{\prime} Q_{0}^{n+2} t^{2 n+2}}{M^{\prime}}\right)^{1 /(3 n+6)}, \quad \epsilon_{m}=\frac{Q_{0} t}{L_{m}^{3}} .
$$

Further, we substitute $L_{m}$ and $\epsilon_{m}$ into the remaining dimensionless groups:

$$
\mathcal{C}_{m}=C^{\prime}\left(\frac{E^{\prime 4} t^{5 n+2}}{M^{\prime 4} Q_{0}^{2 n+4}}\right)^{1 /(6 n+12)}, \mathcal{T}_{m}=\tau_{0}\left(\frac{t^{2 n}}{M^{\prime 2} E^{\prime n}}\right)^{1 /(n+2)}, \quad \mathcal{K}_{m}=K^{\prime}\left(\frac{t^{4 n-2}}{M^{\prime 5} E^{\prime 6 n+7} Q_{0}^{n+2}}\right)^{1 /(6 n+12)}
$$

where we reassign $\mathcal{G}_{c}, \mathcal{G}_{t}, \mathcal{G}_{k}$ as the dimensionless leak-off $\mathcal{C}_{m}$, yield stress $\mathcal{T}_{m}$ and toughness $\mathcal{K}_{m}$. After that, we obtain formulas for the prefactors $R_{m}^{*}, w_{m}^{*}$ :

$$
R_{m}^{*}=\left(2 \pi \beta_{m} \alpha_{m}^{n /(n+2)} \mathcal{B}_{m}\right)^{-1 / 3}, \quad w_{m}^{*}=\left(\frac{\beta_{m}^{2} \alpha_{m}^{2 n /(n+2)}}{2 \pi \mathcal{B}_{m}}\right)^{1 / 3}
$$



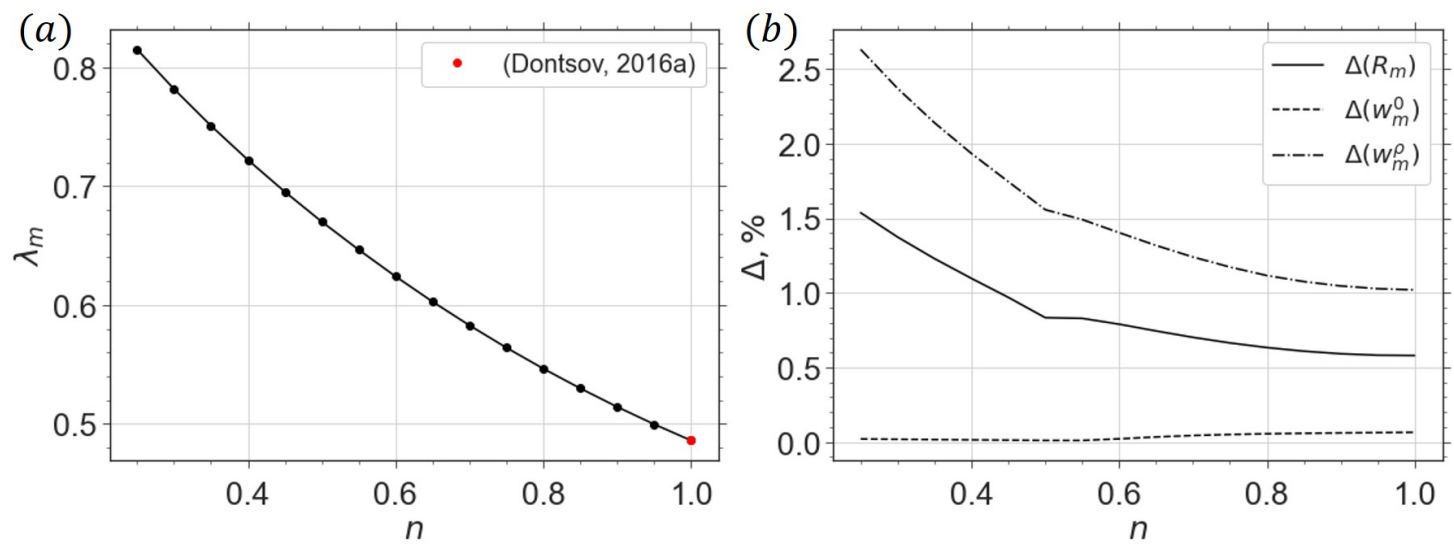

Figure 2: Figure (a) presents the dependence of $\lambda_{m}$ on the flow index $n$. The red dot corresponds to the $\lambda_{m}(1)$ value obtained by Dontsov (2016a). In Figure (b), we demonstrate the relative errors in the calculation of the radius and opening parameters for the $M$-vertex with the help of the simplified approach (Section 3.2).

where $\alpha_{m}=(2 n+2) /(3 n+6), \mathcal{B}_{m}=\mathcal{B}\left(\lambda_{m}, \bar{\delta}_{m}\right)$ (see equation (27)), the value of $\beta_{m}$ is provided by equation (29), and $\bar{\delta}_{m}=2 /(2+n)$ is taken from the storage-viscosity tip asymptote (Dontsov and Kresse, 2018). The parameter $\lambda_{m}$ is obtained by fitting the approximate solution above (34) and (40) to the full numerical solution (Section 3.1) in terms of $R_{m}$ and $w_{m}(0, t)$. We computed $\lambda_{m}$ for different values of flow indexes $n \in[0.25,1]$, and the obtained curve is shown in Figure 2(a). The relative errors defined by equations (35)-(37) are depicted in Figure 2(b), where $\Delta\left(R_{m}\right)$ is shown by the solid line, $\Delta\left(w_{m}^{0}\right)$ is shown by dashed line, and $\Delta\left(w_{m}^{\rho}\right)$ is shown by dash-dotted line.

Figures 3(a) and 3(d) present comparison of the approximate normalised profiles of $w_{m}(\rho, t), p_{m}(\rho, t)$ (dashed lines) with the corresponding properties evaluated by the fully numerical solution (solid lines) for $n=1$ (light-blue colour) and $n=0.3$ (blue colour). We use the normalisation of the width by $\epsilon_{m}(t) L_{m}(t)$, pressure by $\epsilon_{m}(t) E^{\prime}$ as per (32) and of the radial distance from the source by $L_{m}(t)$ scale; these scales account for the $M$ limiting solution dependence on time and largely on the flow behaviour index $n$. The characteristics for the Newtonian fluid are very close to each other, while the accurate and approximate profiles for $n=0.3$ intersect for both opening and pressure; moreover, there is a noticeable difference for the pressure values near the fracture front.

Let us consider the case of the leak-off-viscosity scaling and the corresponding $\tilde{M}$-vertex solution (subscript $\tilde{m}$ ). This scaling corresponds to $\mathcal{G}_{c}=\mathcal{G}_{m}=1$. Hence,

$$
L_{\tilde{m}}=t^{1 / 4} \sqrt{\frac{Q_{0}}{C^{\prime}}}, \quad \epsilon_{\tilde{m}}=\left(\frac{C^{\prime 6} M^{\prime 4 / n}}{Q_{0}^{2} t^{3} E^{\prime 4 / n}}\right)^{\frac{n}{8 n+8}} .
$$

The evolution parameters have the meaning of the dimensionless storage $\mathcal{S}_{\tilde{m}}$, yield stress $\mathcal{T}_{\tilde{m}}$ and toughness $\mathcal{K}_{\tilde{m}}$ :

$$
S_{\tilde{m}}=\left(\frac{M^{\prime 4} Q_{0}^{2 n+4}}{E^{\prime 4} t^{5 n+2} C^{\prime 6(n+2)}}\right)^{1 /(8+8 n)} \quad, \quad \mathcal{T}_{\tilde{m}}=\tau_{0}\left(\frac{Q_{0}^{2} t^{3}}{M^{\prime 4 / n} C^{6} E^{\prime 4}}\right)^{n /(4+4 n)}, \quad \mathcal{K}_{\tilde{m}}=K^{\prime}\left(\frac{C^{\prime 2-4 n} t^{2 n-1}}{M^{\prime 4} Q_{0}^{2} E^{\prime 8 n+4}}\right)^{1 /(8+8 n)} .
$$

For the $\tilde{M}$-vertex, we derive the following prefactors $R_{\tilde{m}}^{*}, w_{\tilde{m}}^{*}$ :

$$
R_{\tilde{m}}^{*}=\frac{\sqrt{2}}{\pi}, \quad w_{\tilde{m}}^{*}=\beta_{\tilde{m}}\left(\frac{\alpha_{\tilde{m}}^{n} 2^{2+3 n}}{\pi^{4+2 n}}\right)^{1 /(4+4 n)},
$$

where $\alpha_{\tilde{m}}=1 / 4, \beta_{\tilde{m}}$ is given by equation (29), and we also utilise $\bar{\delta}_{\tilde{m}}=(4+n) /(4+4 n)$ known from the leak-offviscosity near-tip region asymptote (Dontsov and Kresse, 2018). The parameter $\lambda_{\tilde{m}}$ featured in the $w_{\tilde{m}}$ profile is found from the comparison of $w_{\tilde{m}}(0, t)$ (equation (34) combined with (43)) with the numerical solution (Section 3.1). This 

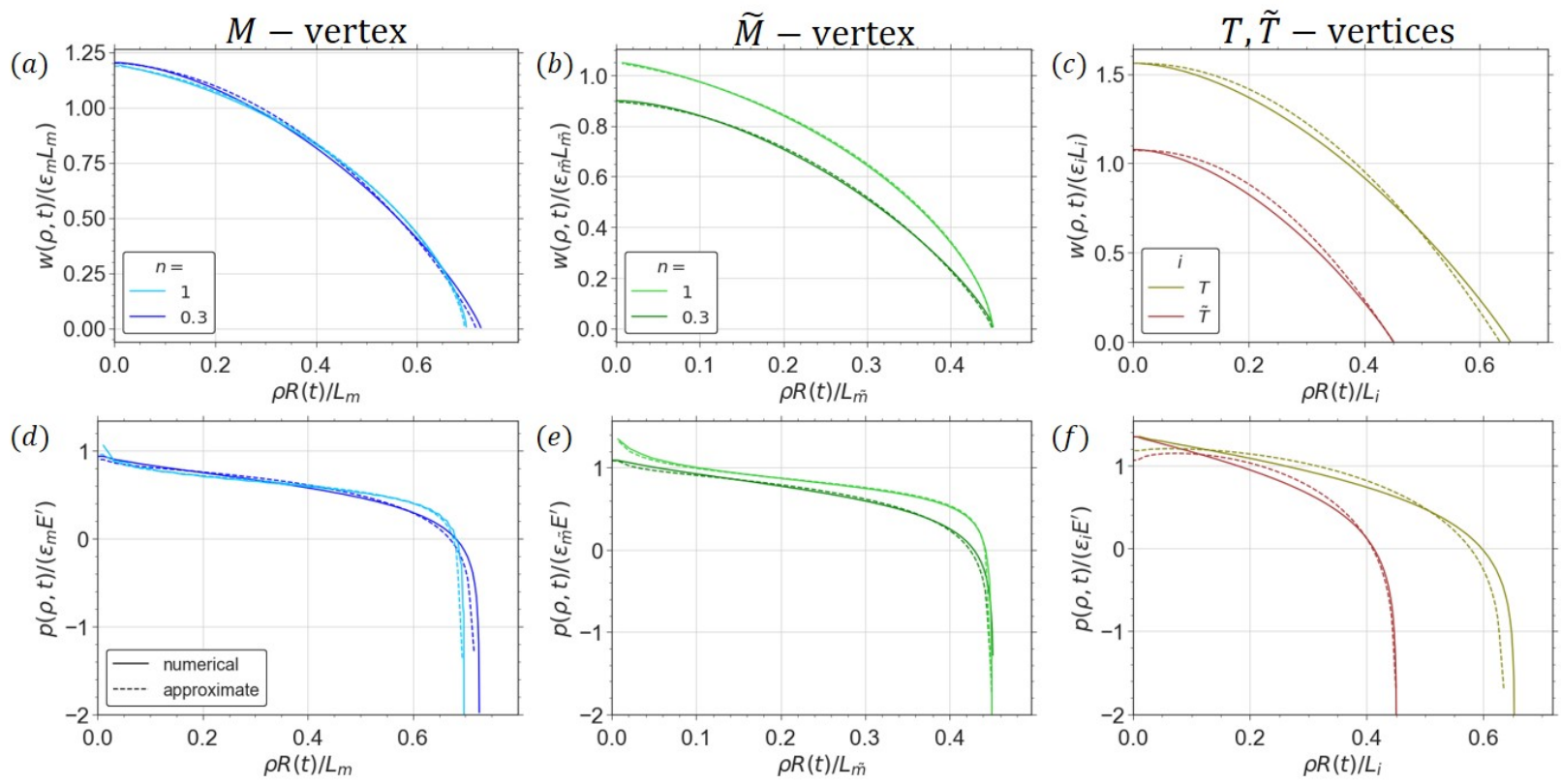

Figure 3: The spatial variations of the crack opening (upper line) and net fluid pressure (bottom line) in the limiting propagation regimes $M$ (panels (a) and (d)), $\tilde{M}$ (panels (b) and (e)), $T$ and $\tilde{T}$ (panels (c) and (f)) calculated by the fully numerical (solid lines) and approximate (dashed lines) approaches. We apply $\epsilon_{i}(t) L_{i}(t), \epsilon_{i}(t) E^{\prime}$ and $L_{i}(t)$ dimensional prefactors for the normalisation of $w(\rho, t), p(\rho, t)$ profiles, and distance from the source, and here, the subscript $i$ denotes the analysed regime. In the case of $M$ and $\tilde{M}$ vertex solutions, we look at $n=1$ or $n=0.3$ and show the computed properties in plots (a), (d) and (b), (e) by the light-blue or light-green and blue or green colours, correspondingly. The normalisation coefficients take into account the value for the flow behaviour index. We use olive and maroon colours for the yield stress dominated regimes $T$ and $\tilde{T}$ in the charts (c), (f).
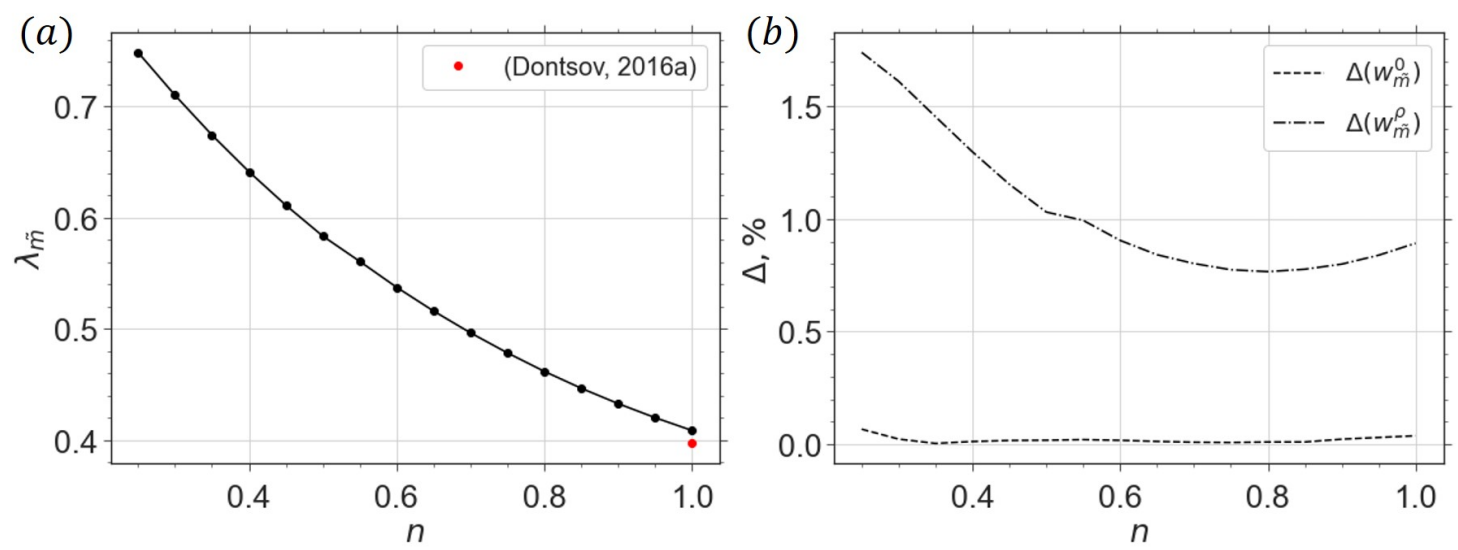

Figure 4: Figure (a) shows the values of the function $\lambda_{\tilde{m}}(n)$ computed numerically. Here, we mark $\lambda_{\tilde{m}}(1)$ obtained by Dontsov (2016a) by the red dot. Figure (b) presents the relative errors in the estimation of the opening profile for the $\tilde{M}$-vertex via the simplified approach (Section 3.2).

approach yields to the values of $\lambda_{\tilde{m}}(n)$ presented in Figure 4(a). Moreover, we also estimate the relative error in the crack aperture values $w_{\tilde{m}}(\rho, t)$ provided by equation (25) with $\lambda_{\tilde{m}}(n)$ (Figure 4(b)), and we utilise equations (36), (37) for $\Delta\left(w_{\tilde{m}}^{0}\right)$ and $\Delta\left(w_{\tilde{m}}^{\rho}\right)$.

In the panels (b), (e) of Figure 3, we demonstrate the spatial variations of the width and pressure calculated by the accurate solution (solid lines) and provided by the approximate approach (dashed lines), i.e., $w_{\tilde{m}}(\rho, t), p_{\tilde{m}}(\rho, t)$, for two 
cases of the flow behaviour index $n=1$ (light-green colour) and $n=0.3$ (green colour). The dimensional prefactors $\epsilon_{\tilde{m}}(t) L_{\tilde{m}}(t), \epsilon_{\tilde{m}}(t) E^{\prime}, L_{\tilde{m}}(t)$ are applied for the normalisation of the aperture, pressure and distance from the source, and they include the value of the flow behaviour index. We can mention that for both analysed values of the flow index, the accurate and approximate opening and pressure profiles are very close to each other.

Next, we discuss the storage-toughness scaling that corresponds to the $K$-vertex solution (subscript " $k$ "). Here, we require $\mathcal{G}_{v}=\mathcal{G}_{k}=1$ leading to:

$$
L_{k}=\left(\frac{E^{\prime} Q_{0} t}{K^{\prime}}\right)^{2 / 5}, \quad \epsilon_{k}=\left(\frac{K^{\prime 6}}{E^{\prime 6} Q_{0} t}\right)^{1 / 5},
$$

and the evolution parameters can be interpreted as the dimensionless leak-off $\mathcal{C}_{k}$, yield stress $\mathcal{T}_{k}$ and viscosity $\mathcal{M}_{k}$ :

$$
\mathcal{C}_{k}=C^{\prime}\left(\frac{E^{\prime 8} t^{3}}{K^{\prime 8} Q_{0}^{2}}\right)^{1 / 10}, \quad \mathcal{T}_{k}=\tau_{0}\left(\frac{E^{\prime 7} Q_{0}^{2} t^{2}}{K^{\prime 12}}\right)^{1 / 5}, \quad \mathcal{M}_{k}=\left(\frac{M^{\prime 5} E^{\prime 6 n+7} Q_{0}^{n+2} t^{2-4 n}}{K^{\prime 6(n+2)}}\right)^{1 /(5 n)}
$$

The LEFM asymptote governs the tip behaviour in the storage-toughness limiting propagation regime, i.e. $\bar{\delta}_{k}=$ $1 / 2$. Moreover, in this case, the crack opening profile is elliptical (Savitski and Detournay, 2002). Consequently, the simplified form of the radius crack opening profile (25) allows describing it accurately by taking $\lambda_{k}=1 / 2$. Using the global fluid balance equation (27) and the "square-root" asymptote (Irvin, 1957), we find out the following coefficient in the radius and opening profiles:

$$
R_{k}^{*}=\left(\frac{3}{\sqrt{2} \pi}\right)^{2 / 5}, \quad w_{k}^{*}=\left(\frac{3}{\sqrt{2} \pi}\right)^{1 / 5}
$$

Next, we construct the leak-off-toughness scaling and focus on the $\tilde{K}$-vertex solution (subscript " $\tilde{k}$ "). We put $\mathcal{G}_{c}=\mathcal{G}_{k}=1$, and get the expressions for the length scale $L_{\tilde{k}}$ and the small parameter $\epsilon_{\tilde{k}}$ :

$$
L_{\tilde{k}}=t^{1 / 4} \sqrt{\frac{Q_{0}}{C^{\prime}}}, \quad \epsilon_{\tilde{k}}=\left(\frac{C^{\prime 2} K^{\prime 8}}{E^{\prime 8} Q_{0}^{2} t}\right)^{1 / 8}
$$

The evolution parameters are identified as the dimensionless storage $S_{\tilde{k}}$, yield stress $\mathcal{T}_{\tilde{k}}$ and viscosity $\mathcal{M}_{\tilde{k}}$ :

$$
S_{\tilde{k}}=\frac{K^{\prime} Q_{0}^{1 / 4}}{C^{\prime 5 / 4} E^{\prime} t^{3 / 8}}, \quad \mathcal{T}_{\tilde{k}}=\tau_{0} \frac{E^{\prime} Q_{0}^{1 / 2} t^{1 / 4}}{C^{\prime 1 / 2} K^{\prime 2}}, \quad \mathcal{M}_{\tilde{k}}=\left(\frac{M^{\prime} Q_{0}^{1 / 2} E^{\prime 1+2 n} C^{\prime n-1 / 2} t^{1 / 4-n / 2}}{K^{\prime 2+2 n}}\right)^{1 / n} .
$$

Similarly to the $K$-limiting propagation regime, the crack opening behaviour near the tip is described by the LEFM asymptote $\left(\bar{\delta}_{\tilde{k}}=1 / 2\right)$, while the whole profile is elliptical $\left(\lambda_{\tilde{k}}=1 / 2\right)$. The derivation of the prefactors for the radius and opening leads to:

$$
R_{\tilde{k}}^{*}=\frac{\sqrt{2}}{\pi}, \quad w_{\tilde{k}}^{*}=\frac{2^{1 / 4}}{\sqrt{\pi}} .
$$

Finally, next we reach the cases that correspond to dominance of yield stress. The storage-yield-stress scaling (subscript " $t$ ") corresponds to the case: $\mathcal{G}_{t}=\mathcal{G}_{m}=1$, which results to:

$$
L_{t}=\left(\frac{t E^{\prime 1 / 2} Q_{0}}{\tau_{0}^{1 / 2}}\right)^{1 / 3}, \quad \epsilon_{t}=\sqrt{\frac{\tau_{0}}{E^{\prime}}}
$$

In turn, the evolution parameters are the dimensionless leak-off, viscosity and toughness:

$$
\mathcal{C}_{t}=C^{\prime}\left(\frac{E^{\prime} \sqrt{t}}{Q_{0} \tau_{0}}\right)^{1 / 3}, \quad \mathcal{M}_{t}=\frac{\sqrt{E^{\prime}} M^{\prime 1 / n}}{t \tau_{0}^{(n+2) /(2 n)}}, \quad \mathcal{K}_{t}=\frac{K^{\prime}}{\left(E^{\prime 7} Q_{0}^{2} t^{2} \tau_{0}^{5}\right)^{1 / 12}}
$$


Further, we should determine the prefactors $R_{t}^{*}, w_{t}^{*}$ from the global fluid balance (27) and the yield stress dominated near-tip region asymptote (Bessmertnykh and Dontsov, 2019):

$$
R_{t}^{*}=\frac{1}{2^{5 / 6} \sqrt{\pi} \mathcal{B}_{t}^{1 / 3}}, \quad w_{t}^{*}=\frac{2^{2 / 3}}{\mathcal{B}_{t}^{1 / 3}},
$$

where $\mathcal{B}_{t}=\mathcal{B}\left(\lambda_{t}, \bar{\delta}_{t}\right)$ and $\bar{\delta}_{t}=1$. Using the radius and wellbore opening characteristics computed via the accurate numerical solution (Section 3.1), we find out that $\lambda_{t}=1.029$ provides the most accurate approximation. This value together with equations (25), (52) result in $\Delta\left(R_{t}\right)=3 \%$ (equation (35)) while the $w_{t}(0, t)$ is captured precisely. At the same time, the absolute difference between the fully numerical and approximate opening profiles $w_{t}(\rho, t)$ normalised by the opening at the wellbore $w_{t}(0, t)$ can reach $\Delta\left(w_{t}^{\rho}\right)=5 \%$ (equation (37)), which demonstrates that the accuracy of the approximation (34) reduces for such values of $\lambda$.

The last case is the leak-off-yield-stress scaling and the $\tilde{T}$-vertex solution associated with it. We set $\mathcal{G}_{c}=\mathcal{G}_{t}=1$, which results in the following parameters:

$$
L_{\tilde{t}}=t^{1 / 4} \sqrt{\frac{Q_{0}}{C^{\prime}}}, \quad \epsilon_{\tilde{t}}=\sqrt{\frac{\tau_{0}}{E^{\prime}}} .
$$

Here, the evolution parameters are identified as the dimensionless storage $S_{\tilde{t}}$, viscosity $\mathcal{M}_{\tilde{t}}$ and toughness $\mathcal{K}_{\tilde{t}}$ :

$$
S_{\tilde{t}}=\left(\frac{Q_{0} \tau_{0}}{C^{\prime 3} E^{\prime} \sqrt{t}}\right)^{1 / 2}, \quad \mathcal{M}_{\tilde{t}}=\frac{C^{\prime 3 / 2} E^{\prime} M^{\prime 1 / n}}{\sqrt{Q_{0}} t^{3 / 4} \tau_{0}^{(n+1) / n}}, \quad \mathcal{K}_{\tilde{t}}=K^{\prime}\left(\frac{C^{\prime}}{E^{\prime 2} Q_{0} \sqrt{t} \tau_{0}^{2}}\right)^{1 / 4} .
$$

Then, we retrieve the coefficients for the radius and opening (equation (34)) based on the global fluid balance (27) and the tip asymptote (Bessmertnykh and Dontsov, 2019):

$$
R_{\tilde{t}}^{*}=\frac{\sqrt{2}}{\pi}, \quad w_{\tilde{t}}^{*}=\frac{4}{\sqrt{\pi}} .
$$

Similarly to the $\tilde{M}$-vertex solution, we estimate $\lambda_{\tilde{t}}$ via the tuning of the simplified opening profile (25) to the accurate numerical solution (Section 3.1). We compute $\lambda_{\tilde{t}}=1.077$, and it provides the best approximation which reproduces precisely the fracture opening at the wellbore $w_{\tilde{t}}(0, t)$ and gives the error in the spatial variation $w_{\tilde{t}}(\rho, t)$ (in relation to $\left.w_{\tilde{t}}(0, t)\right)$ up to $\Delta\left(w_{\tilde{t}}^{\rho}\right)=6 \%$ (equation (37)).

Figures 3(c) and 3(f) depict the aperture and pressure profiles obtained by the accurate method (solid lines) and that computed by the approximate one (dashed lines) for the yield stress dominated regimes $T$ (olive colour) and $\tilde{T}$ (maroon colour). For both vertices, we observe the intersection between the fully numerical and simplified solutions and a noticeable difference between them, which is caused by the loss of accuracy of the width approximation utilised to construct the simplified solution (34). One possible mitigation of this issue is to introduce a more sophisticated expression for fracture width that captures such behaviour for the $T$ and $\tilde{T}$ limits. The existing approximation was initially proposed for smaller values of $\bar{\delta}$ and $\lambda$ that correspond to Newtonian fluids. However, for the purpose of this study, i.e., for exploring the problem parameter space, such accuracy is acceptable, especially since the boundaries of the limiting solutions vary on a logarithmic scale.

The fracture characteristics observed in the limiting propagation regimes are summarised in Appendix B. In addition to the dimensional form, we also present the solutions in the normalised form obtained by the application of the $m k$-scaling introduced in Section 5.1.

\subsection{Representation of the problem solution space}

The parametric space of solutions for the discussed radial fracture model can be conceptually presented in the form of a hexahedral pyramid, and the limiting propagation regimes are located at its vertices. The sketch of the pyramid $M \tilde{M} \tilde{K} K T \tilde{T}$ is shown in Figure 5. Each edge of the pyramid links two vertex solutions, e.g., $i$ and $j$, and it is possible to introduce a characteristic transition time between them $t_{i j}$ by solving the following equation: $\epsilon_{i}(t)=\epsilon_{j}(t) \rightarrow t=t_{i j}$. Furthermore, the evolution along the edge is controlled by a single evolution parameter, which can be expressed as a 


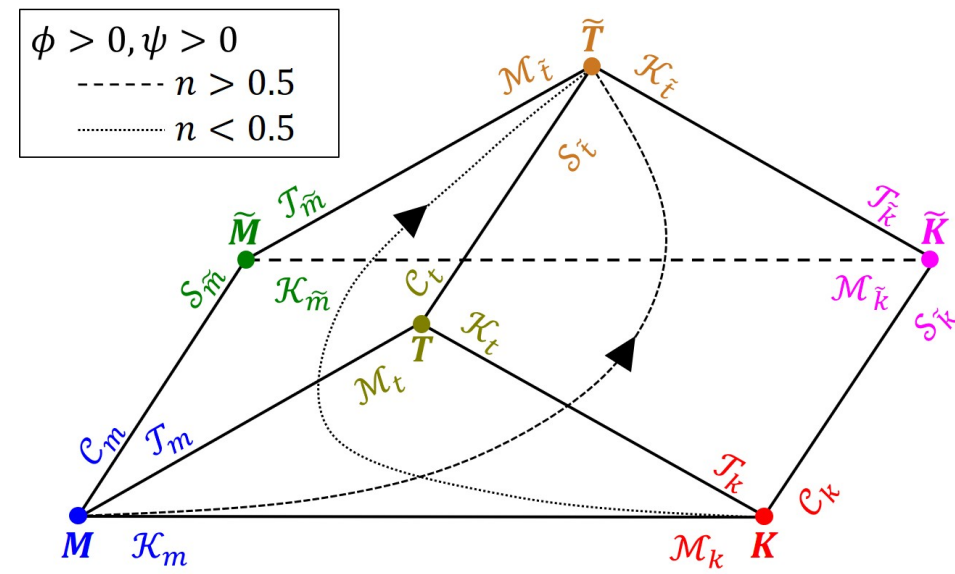

Figure 5: Parametric space $M \tilde{M} \tilde{K} K T \tilde{T}$ for a radial hydraulic fracture in a permeable rock driven by a fluid with HerschelBulkley rheology. The vertex solutions are located at its corners. The evolution parameters are specified near the edges for each propagation regime. The solution trajectories examples in the general case $(\phi>0, \psi>0)$ are depicted for two values of the flow index: $n>0.5$ (dashed line) and $n<0.5$ (dotted line).

function of normalised time $\tau_{i j}=t / t_{i j}$ (two evolution parameters are related to each edge). Let us consider the $M T$ edge as an example. The transition time scale is $t_{m t}=\sqrt{E^{\prime}} M^{\prime 1 / n} \tau_{0}^{-(n+2) /(2 n)}$, while $\mathcal{T}_{m}(t)$ and $\mathcal{M}_{t}(t)$ are evolution parameters related to this edge. By introducing the dimensionless time $\tau_{m t}=t / t_{m t}$, we can write out the relations: $\mathcal{T}_{m}=\tau_{m t}^{2 n /(n+2)}, \mathcal{M}_{t}=\tau_{m t}^{-1}$.

In the current problem, we have 9 transition time scales, and only 3 of them are independent, e.g., $t_{m k}, t_{m \tilde{m}}, t_{m t}$. Consequently, location of the solution inside the parametric space can be expressed as a function of the three dimensionless times $\tau_{m k}=t / t_{m k}, \tau_{m \tilde{m}}=t / t_{m \tilde{m}}, \tau_{m k}=t / t_{m t}$. Further, we introduce the parameters $\phi$ and $\psi$ as:

$\phi=\left(\frac{t_{m k}}{t_{m \tilde{m}}}\right)^{(10 n+4) /(3 n+6)}=C^{\prime 4}\left(\frac{M^{\prime 3} E^{\prime 10 n+1} Q_{0}^{2-n}}{K^{\prime 10 n+4}}\right)^{1 /(2 n-1)} \quad, \quad \psi=\left(\frac{t_{m k}}{t_{m t}}\right)^{2 n /(n+2)}=\tau_{0}\left(\frac{E^{\prime 4} Q_{0} M^{\prime 1 / n}}{K^{\prime 6}}\right)^{n /(2 n-1)}$,

and they can be interpreted as the dimensionless leak-off and yield stress numbers. By taking the set of parameters $\left\{\tau=\tau_{m k}, \phi, \psi\right\}$, we can express $\tau_{m \tilde{m}}$ and $\tau_{m t}$ in terms of them in order to characterise the solution trajectories. Along each trajectory, the numbers $\phi$ and $\psi$ are constant while $\tau$ varies.

When the flow index is greater than one-half $(n>0.5)$, all solution trajectories start from the $M$ limiting propagation regime (early-time asymptote) since all the dimensionless groups (39) vanish when time tends to zero. However, their destination points (large-time asymptote) can be different: (i) $\phi=\psi=0-K$-vertex, (ii) $\phi>0, \psi=0$ $\tilde{K}$-vertex, (iii) $\phi=0, \psi>0-T$-vertex and (iv) $\phi>0, \psi>0-\tilde{T}$-vertex. One can explain the endpoints of the solution trajectories by looking at the evolution parameters of the corresponding regimes (e.g., (45), (48), (51), (54)) which go to zero when time tends to infinity or they are identically zero due to values of $\phi, \psi$. For intermediate time intervals, the solution trajectory can be attracted to either of the vertices, and its behaviour strongly depends on the values of $\phi$ and $\psi$. For example, when the yield stress is absent $(\psi=0), K$ and $\tilde{M}$ regimes can be considered in the general solution as intermediate asymptotes when the leak-off is small $(\phi \ll 1)$ and large $(\phi \gg 1)$, correspondingly. As another example, we can take zero leak-off case $(\phi=0)$ with the yield stress $(\psi>0)$, and $K$-vertex attracts the solution trajectory when $\psi \ll 1$.

The situation changes for $n<0.5$. Here, the trajectories start at the $K$-vertex (see the corresponding evolution parameters (45) turn to zero when $t=0$ ), and the final point can be: (i) $\phi=\psi=0-M$-vertex, (ii) $\phi>0, \psi=0-$ $\tilde{M}$-vertex, (iii) $\phi=0, \psi>0-T$-vertex and (iv) $\phi>0, \psi>0-\tilde{T}$-vertex (similarly to the previous discussion, it is required to draw attention to the parameters provided by equations (39), (42), (51), (54)).

We want to emphasise that in the general case when the dimensionless numbers $\phi$ and $\psi$ are both non-zero, the large time asymptotic behaviour is always dominated by leak-off and yield stress, i.e., $\tilde{T}$-vertex, regardless of the value 
of the flow index $n$. The examples of the solution trajectories for $n>0.5$ and $n<0.5$ are shown in Figure 5 by dashed and dotted lines, respectively.

Finally, we should mention that the chosen set of parameters, i.e. $\left\{\tau=\tau_{m k}, \phi, \psi\right\}$, is not applicable for the description of the solution trajectories behaviour for $n=0.5$ since they are not properly defined in this case. One possibility to mediate this issue is to introduce another set of variables, e.g.,

$\left\{\tau=\tau_{m \tilde{m}}, \phi^{\prime}=\left(t_{m k} / t_{m \tilde{m}}\right)^{(1-2 n) /(3 n+6)}, \psi^{\prime}=\left(t_{m \tilde{m}} / t_{m t}\right)^{2 n /(n+2)}\right\}$, where $\phi^{\prime}$ is the dimensionless toughness, and $\psi^{\prime}$ is the dimensionless yield stress. Nevertheless, since the problematic region consists of solely a single point, we proceed with the chosen set of parameters $\left\{\tau=\tau_{m k}, \phi, \psi\right\}$.

\section{Results and discussion}

\subsection{Admissible ranges of the dimensionless problem parameters.}

Let us start by rewriting the problem formulation in terms of the dimensionless variables. For the normalisation of the governing equations, we rely on the $m k$-scaling consisting on the following parameters (Detournay, 2016):

$$
\rho=\frac{r}{R}, \quad \tau=\frac{t}{t_{m k}}, \quad \gamma=\frac{R}{L_{m k}}, \quad \Omega=\frac{w}{\epsilon_{m k} L_{m k}}, \quad \Pi=\frac{p}{E^{\prime} \epsilon_{m k}},
$$

where $t_{m k}$ is the transition timescale between $M$ and $K$ limiting regimes (Section 4.2), $L_{m k}=L_{m}\left(t_{m k}\right)$ is the lengthscale, and $\epsilon_{m k}=\epsilon_{m}\left(t_{m k}\right)$ is the small parameter (see equation (38)):

$$
t_{m k}=\left(\frac{M^{\prime 5} E^{\prime 6 n+7} Q_{0}^{n+2}}{K^{\prime 6(n+2)}}\right)^{1 /(4 n-2)}, \quad L_{m k}=\left(\frac{M^{\prime} E^{\prime 2 n+1} Q_{0}^{n}}{K^{2(n+1)}}\right)^{1 /(2 n-1)}, \quad \epsilon_{m k}=\left(\frac{K^{\prime 6 n}}{M^{\prime} E^{\prime 6 n-1} Q_{0}^{n}}\right)^{1 /(4 n-2)} .
$$

The elasticity (13), Reynolds (16), (17), global fluid balance (18) equations and the propagation condition (19) written in terms of the dimensionless distance $\rho$ and using (57) and (58) can be written in the normalised form as:

- Elasticity:

$$
\Pi=-\frac{1}{2 \pi \gamma} \int_{0}^{1} G(\rho, s) \frac{\partial \Omega}{\partial s} d s
$$

- Reynolds:

$$
\begin{aligned}
& \frac{\partial \Omega}{\partial \tau}-\frac{\rho \dot{\gamma}}{\gamma} \frac{\partial \Omega}{\partial \rho}+\frac{\phi^{1 / 4}}{\sqrt{\tau-\theta_{0}}}= \\
& =\frac{1}{\gamma \rho} \frac{\partial}{\partial \rho}\left[\rho \frac{\Omega^{2+1 / n}}{\gamma^{1 / n}} \frac{\partial \Pi}{\partial \rho}\left|\frac{\partial \Pi}{\partial \rho}\right|^{1 / n-1}\left(1-2 \psi \frac{\gamma}{\Omega}\left|\frac{\partial \Pi}{\partial \rho}\right|^{-1}\right)^{1+1 / n}\left(1+2 \psi \frac{\gamma}{\Omega}\left|\frac{\partial p}{\partial \rho}\right|^{-1} \frac{n}{n+1}\right)\right],
\end{aligned}
$$

where the dimensionless leak-off $\phi$ and yield stress $\psi$ are defined in equation (56), and the dimensionless inverse radius function is given by $\theta_{0}(\rho, \tau)=t_{0}(r) / t_{m k}$. For completeness, we also provide the definitions of $\phi$ and $\psi$ through the characteristics of the $m k$-scaling:

$$
\phi=\left(\frac{C^{\prime} \sqrt{t_{m k}}}{L_{m k} \epsilon_{m k}}\right)^{4}, \quad \psi=\frac{\tau_{0}}{E^{\prime} \epsilon_{m k}^{2}}
$$

- Global fluid balance:

$$
\frac{\tau}{\pi \gamma^{2}}=2 \int_{0}^{1} \rho \Omega d \rho+4 \phi^{1 / 4} \int_{0}^{1} \rho \sqrt{\tau-\theta_{0}} d \rho
$$

- Propagation condition:

$$
\Omega=\sqrt{\gamma} \sqrt{1-\rho}, \quad \rho \rightarrow 1
$$


We can use the numerical schemes presented in Sections 3.1 and 3.2 for the solution of the normalised system of governing equations assuming $E^{\prime}=K^{\prime}=M^{\prime}=Q_{0}=1, C^{\prime}=\phi^{1 / 4}, \tau_{0}=\psi$, and $t=\tau$.

Further, it is useful to estimate the realistic ranges of the dimensionless governing parameters $\phi$ and $\psi$. To obtain the desired ranges, some input parameters are kept fixed, while the remaining ones are altered within their own intervals. We consider the following values/spans:

- geomechanics:

○ plane-strain elastic modulus: $E^{\prime}=10 \div 50 \mathrm{GPa}$;

○ rock toughness: $K_{I c}=0.5 \div 2.5 \mathrm{MPa} \cdot \sqrt{\mathrm{m}}$;

○ far-field confining stress: $\sigma_{o}=10 \div 30 \mathrm{MPa}$;

- reservoir:

○ permeability: $k=0 \div 100 \mathrm{mD}$;

○ porosity: $\phi_{r}=5 \div 25 \%$;

○ ratio of far-field pore pressure and far-field confining stress: $p_{o} / \sigma_{o}=0.4 \div 0.95$;

- Fracturing fluid:

○ flow behaviour index: $n=0.5 \div 1$;

○ consistency index: $M=10^{-3} \div 2 \mathrm{~Pa} \cdot \mathrm{s}^{\mathrm{n}}$;

○ yield stress: $\tau_{0}=0 \div 15 \mathrm{~Pa}$;

- pore fluid:

○ viscosity: $\mu=1 \div 5 \mathrm{cP}$;

○ total compressibility: $c_{t}=10^{-3} \mathrm{MPa}^{-1}$;

- volumetric injection rate:

○ $Q_{0}=0.01 \div 0.1 \mathrm{~m}^{3} / \mathrm{s}$.

We take three values of the flow behaviour index $n=\{0.6,0.8,1\}$ and compute the intervals for the governing parameters $\phi$ and $\psi$ by varying each of the aforementioned dimensional input parameters independently. We illustrate the evaluated domains in Figure 6(a). It can be noticed that the domain for $n=0.6$ (green one) includes the analogous zone for $n=0.8$ (red one) which, in turn, includes the region for $n=1$ (blue one). To understand the structure of the non-dimensional parametric domain for a fixed $n$ (how different dimensional input parameters affect the locations inside it) we look closely at the example of the Bingham fluid $(n=1)$ with the yield stress $\tau_{0}=15 \mathrm{~Pa}$ (yellow decagon in Figure 6(b); such decagons for different $\tau_{0} \in[0,15 \mathrm{~Pa}]$ fill completely the whole blue domain). We identify two sub-domains (octagons) corresponding to the minimum and maximum values of the plane-strain elastic modulus and frame them by coloured dash-dotted lines (Figure 6(b)); the octagons belonging to the intermediate values of $E^{\prime}$ are located between them. Consequently, the resultant interval for the dimensionless leak-off number is controlled by the predefined range for $E^{\prime}$, while the interval for $\psi$ is primary governed by the yield stress parameter and then by $E^{\prime}$. Next, each octagon is limited by two hexagons corresponding to the limiting values of the rock toughness. In Figure 6(c), we show the octagon related to the minimum value of the plane-stain elastic modulus $\left(E^{\prime}=10 \mathrm{GPa}\right)$ by the orange colour and highlight the sub-domains for the minimum and maximum values of $K_{I c}$ by the coloured dashed lines. One can also examine the interior of each hexagon and reveal the parallelograms corresponding to the extreme values of the consistency index and rock permeability which we emphasise by the coloured dotted boundaries and the coloured line fill in Figure 6(d) using the hexagon for $K_{I c}=2.5 \mathrm{MPa} \cdot \sqrt{\mathrm{m}}$. Finally, we can mention that the lower and upper sides of each parallelograms (Figure 6(d)) are locuses of the points represented by the minimum and maximum values of the volumetric fluid injection rate, and the structure of each side includes the alterations of the remaining parameters $\left(\sigma_{o}, \phi_{r}, p_{o} / \sigma_{o}\right)$. 

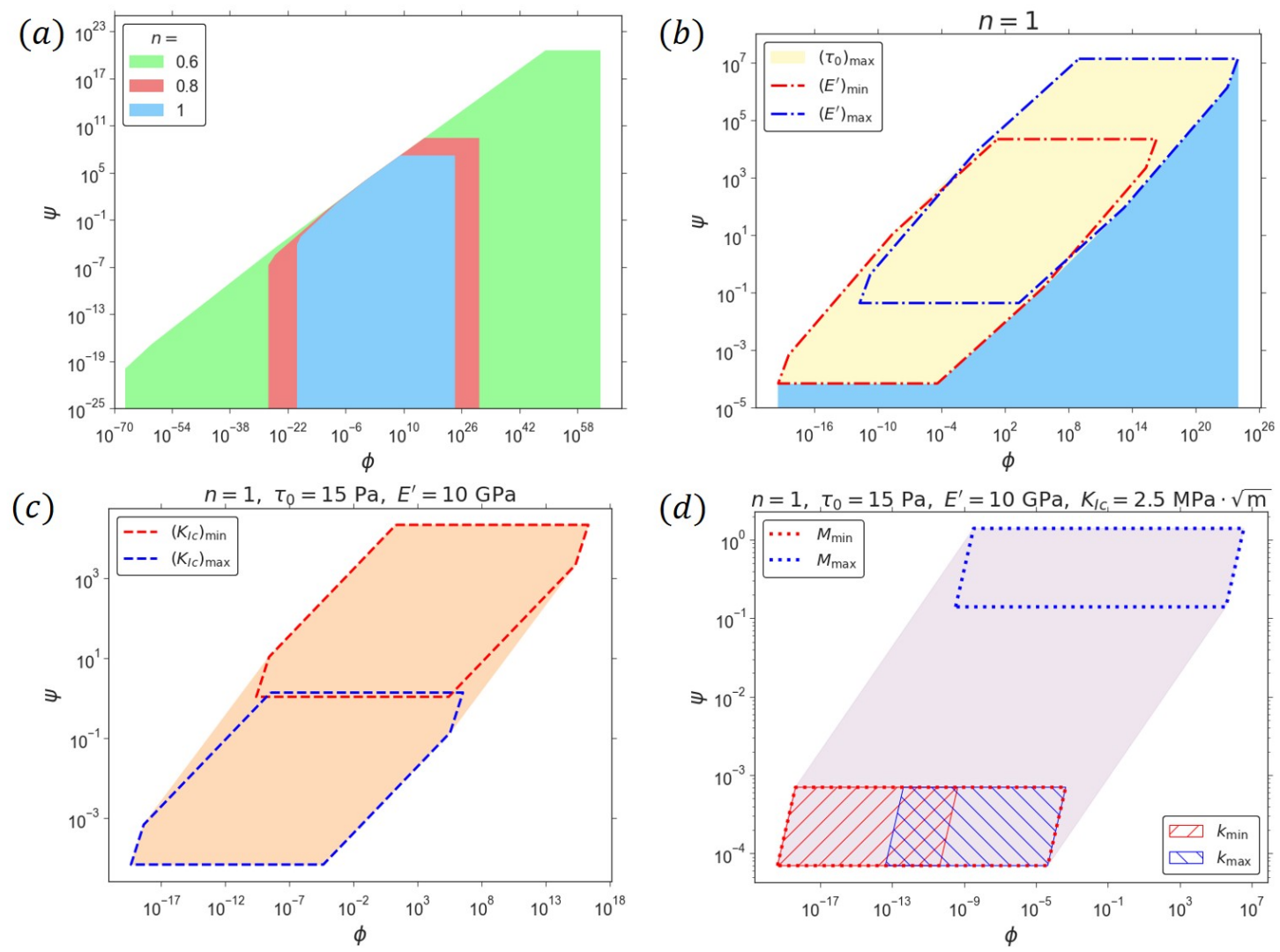

Figure 6: The panel (a) provides the domains in the coordinates $(\phi, \psi)$ corresponding to $n=\{0.6,0.8,1\}$ and typical field parameters by green, red, and blue colours. In the panel (b), the yellow decagon shows the locus for $n=1$ and $\tau_{0}=15$ Pa, while the octagons framed by the red and blue dash-dotted lines are related to the minimum and maximum values of the plane-strain elastic modulus. The structure of the octagon cell is presented in the panel (c) (for $\left.E^{\prime}=10 \mathrm{GPa}\right)$ where the red and blue dashed lines limit the hexagons corresponding to the minimum and maximum values of rock toughness. On the chart (d), we analyse the internal structure of the hexagon (using the case for $K_{I c}=2.5 \mathrm{MPa} \cdot \sqrt{\mathrm{m}}$ ) by identifying the locations of data points belonging to the minimum and maximum values of the consistency index (red and blue dotted boundaries) and rock permeability (domains with red and blue line fill).

\subsection{Analysis of the parametric space}

This section considers analysis of the parametric space for a radial crack driven by a fluid with Herschel-Bulkley rheology. We utilise the dimensionless problem formulation (Section 5.1) in which the crack characteristics governed by the following dimensionless variables: time $(\tau)$, distance from the source $(\rho)$, leak-off $(\phi)$ and yield stress $(\psi)$.

In this section, we focus on the results related to the following values of the flow behaviour index: $n=1$ and $n=0.3$, and the examination includes the following two main components. Firstly, we identify the applicability domains of the limiting propagation regimes inside the parameter space and draw them as the regime maps. The construction of such kind of maps helps to understand the crack propagation conditions for the given problem parameters. The validity zones of the vertices are defined according to the following criterion (the same was used in Dontsov (2016a)):

$$
\sqrt{\left(1-\frac{\Omega_{i}(0, \tau)}{\Omega(0, \tau)}\right)^{2}+\left(1-\frac{\gamma_{i}(\tau)}{\gamma(\tau)}\right)^{2}}<0.01, \quad i=M, \tilde{M}, K, \tilde{K}, T, \tilde{T} .
$$

Equation (59) states that the combination of the relative differences of the opening at the wellbore and of the fracture radius between the numerical solution and the $i$-th limiting regime should be less than $1 \%$ for the solution being considered at the vertex.

It is important to highlight that for the purpose of this section we apply the simplified approach (Section 3.2) for calculations due to its computational efficiency. In Appendix C, we present validation of the corresponding approximate 
Table 1

The validity boundaries of the limiting regimes obtained by setting $\tau_{\text {edge }}=$ const.

\begin{tabular}{|c|c|}
\hline Transition & $\tau_{\text {edge }}=$ \\
\hline$M K$ & $\tau$ \\
\hline$M \tilde{M}$ & $\tau \phi^{(3 n+6) /(10 n+4)}$ \\
\hline$M T$ & $\tau \psi^{(2+n) /(2 n)}$ \\
\hline$K \tilde{K}$ & $\tau \phi^{5 / 6}$ \\
\hline$K T$ & $\tau \psi^{5 / 2}$ \\
\hline$\tilde{M} \tilde{K}$ & $\tau / \sqrt{\phi}$ \\
\hline$\tilde{M} \tilde{T}$ & $\tau \psi^{(4 n+4) /(3 n)} / \sqrt{\phi}$ \\
\hline$\tilde{K} \tilde{T}$ & $\psi^{4} \tau / \sqrt{\phi}$ \\
\hline$T \tilde{T}$ & $\tau \phi^{3 / 2} / \psi^{2}$ \\
\hline
\end{tabular}

solution by comparing its predictions to that provided by the accurate numerical method (Section 3.1).

We apply criterion (59) to estimate the applicability boundaries of all vertex solutions numerically. Moreover, to provide quick analytic estimates, we fit the obtained points by the appropriate analytical functional dependencies that are derived from the consideration of the transition timescales between the limiting regimes. As an example, let us consider the validity boundaries of the storage-viscosity $(M)$ and storage-yield-stress $(T)$ regimes framing the $M T$ transition (another interpretation of the transition is the $M T$-edge of the solution space outlined in Section 4.2). The time $t$ normalised by the transition timescale $t_{m t}$ has the form: $\tau_{m t}=\tau \psi^{(2+n) /(2 n)}$, providing the following relation for the discussed boundaries: $\psi \propto \tau^{-2 n /(2+n)}$. The prefactors are estimated numerically via the fitting procedure for $M$ and $T$ vertices separately. We summarise all possible relationships arising in the model as the applicability boundaries of the limiting solutions in Table 1.

The second element of the analysis is the examination of the variations of the main time-dependent crack characteristics such as radius $\gamma(\tau)$, opening at the wellbore $\Omega(0, \tau)$, pressure at the half-radius $\Pi(1 / 2, t)$, and efficiency $\eta(\tau)$ with the dimensionless leak-off and yield stress parameters.

\subsubsection{Impermeable rock \\ Bingham fluid $(n=1)$}

We start with a radial crack driven by the Bingham fracturing fluid $(n=1)$ in an impermeable formation $(\phi=0)$. The regime map is shown in Figure 7(a) in the coordinates $(\tau, \psi)$. Generally, when the leak-off number $\phi=0$, only three storage vertices can be realised: viscosity $(M)$, toughness $(K)$, and yield stress $(T)$. For the flow behaviour index $n>0.5$, the problem solution evolves from $M$ to $T(\psi>0)$ or to $K(\psi=0)$ as it is confirmed by Figure 7(a) for $n=1$. Moreover, for $n>0.5$ and non-zero yield stress, the solution passes through the toughness dominated regime $(K)$ over an intermediate time range if yield stress $(\psi)$ is small enough. For example, the $K$-vertex approximates the general solution along certain time intervals when $\psi \lesssim 10^{-6}$ for $n=1$, while the solution is dominated by dissipation in the fluid at all times, i.e., solution is given by $M$ to $T$ transition bypassing $K$-vertex, when the yield stress is large enough, e.g., $\psi \gtrsim 1$ for $n=1$ (Figure 7(a)). The time domain of the storage-viscosity regime gradually shrinks with increasing $\psi \gtrsim 10^{-1}$ for $n=1$ (Figure 7(a), whereas the time domain of the storage-yield-stress regime expands.

Further, we look at the solution trajectories in the parameter space for $n=1$ and $\phi=0$ corresponding to the following values of the dimensionless yield stress number: $\psi=\left\{10^{-10}, 10^{-5}, 1,10^{5}\right\}$ (the grey dash-dotted lines in Figure 7(a)). We calculate evolution of the time-dependent crack parameters (radius $\gamma(\tau)$, wellbore opening $\Omega(0, \tau)$, and pressure $\Pi(1 / 2, \tau))$ and plot them normalised by the $M$-vertex solution in Figures $7(\mathrm{~b})-(\mathrm{d})$. One can notice that the solutions are approximately independent of the yield stress $\psi$ and given by the $M$-vertex solution during an initial time period which duration depends on the magnitude of $\psi$. After that initial propagation stage, the presence of the non-zero yield stress leads to a radial crack with the smaller radius (slower fracture growth), larger wellbore opening and higher pressure compared to the $\psi=0$ case (see panels (b) - (d) in Figure 7 where the solution for $\psi=10^{-10}$ coincides with the $\psi=0$ case within the chosen time interval). Moreover, the relative differences between the computed properties for Newtonian $(\psi=0)$ and Bingham $\psi>0$ fluids grow with the increase of the magnitude of the dimensionless yield stress number $\psi$. Various asymptotic regimes of the solution as discussed above with the help of the parametric map (Figure 7(a)) can also be identified with corresponding $M, K$ and $T$ limiting solutions (Appendix B) shown in Figures 7(b) - (d) by coloured dotted lines. 

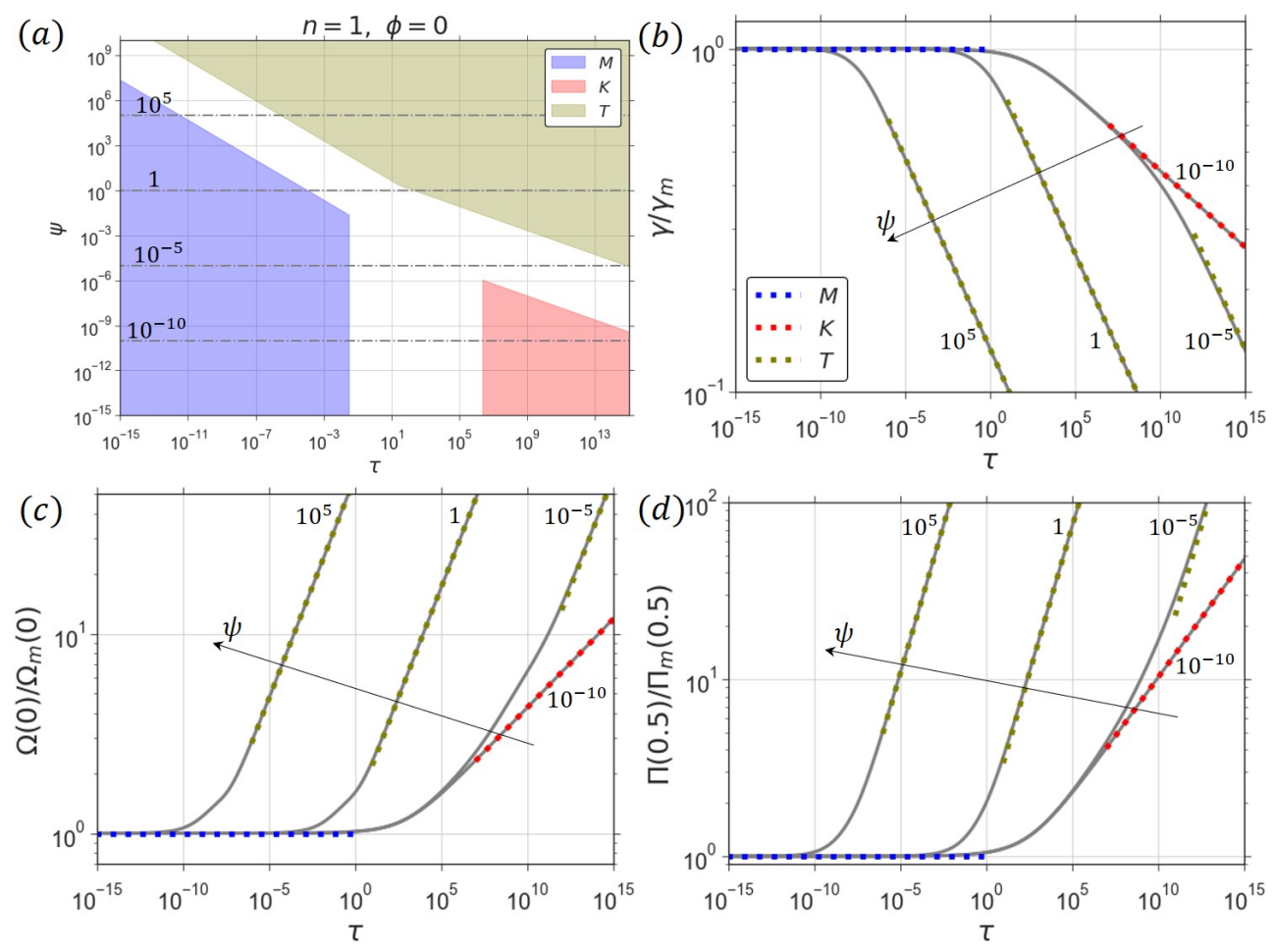

Figure 7: Results for a radial hydraulic fracture driven by the Bingham fluid $(n=1)$ with non-zero yield stress $(\psi>0)$ in an impermeable rock $(\phi=0)$. The regime map $(\mathrm{a})$ is presented in the coordinates $(\tau, \psi)$, and the coloured regions denote the applicability domains of the limiting regimes. The time-dependent crack characteristics (radius (b), opening at the wellbore (c), and pressure at the half-radius (d)) normalised by the storage-viscosity limiting solution ( $M$ ) are depicted for the yield stress number values: $\psi=\left\{10^{-10}, 10^{-5}, 1,10^{5}\right\}$. In figure (a), the grey dash-dotted lines highlight the considered solution trajectories, while the coloured dotted lines in (b) - (d) correspond to the limiting solutions.

\section{Herschel-Bulkley fluid with $n=0.3$}

Now we proceed with the discussion of a radial crack driven by the Herschel-Bulkley fluid with $n=0.3$ in an impermeable rock. The problem solution is demonstrated in Figure 8. For $n<0.5$, the solution trajectories start at the storage-toughness $(K)$ regime and finish at the storage-viscosity $(M)$ regime for the Newtonian fluid $(\psi=0)$ or storage-yield-stress $(T)$ regime for the Herschel-Bulkley fluid $(\psi>0)$ (panel (a) in Figure 8). The general solution can be approximated by the $M$-vertex solution during the intermediate time intervals for $\psi>0$, e.g., it is realised along certain time ranges when $\psi \lesssim 10^{-4}$ for $n=0.3$. The validity zone of the storage-toughness limiting solution $(K)$ diminishes with increasing yield stress number $\psi$, while the $T$-vertex domain expands. Similarly to the Bingham fluid case, we examine four solution trajectories inside the parameter space for $n=0.3$ and $\phi=0$ corresponding to the set of values of the yield stress number: $\psi=\left\{10^{-10}, 10^{-5}, 1,10^{5}\right\}$ (the grey dash-dotted lines in Figure 8(a)), and plot time-dependent crack characteristics normalised by the storage-viscosity limiting solution in Figures $8(\mathrm{~b})-(\mathrm{d})$. The results demonstrate that the non-zero yield stress affects radial fracture growth qualitatively similar to the Bingham fluid case: (i) the non-zero yield stress impacts the crack properties after a definite time range from the initiation which duration is governed by the yield stress number value; (ii) when the solution for the Herschel-Bulkley fluid deviates from that of for the Newtonian fluid, it is characterised by the reduced crack radius and increased maximum opening and pressure; (iii) the relative difference between the compared solutions, i.e., for $\psi>0$ and $\psi=0$, grows with increasing $\psi$.

\subsubsection{Permeable rock}

Next, consider the radial crack problem in a permeable reservoir $(\phi>0)$. Here, we vary both governing parameters $\phi$ and $\psi$ to fully explore the problem parameter space. For each selected value of the flow behaviour index, i.e., $n=1,0.3$, we carry out calculations for the following values of the dimensionless yield stress number: 

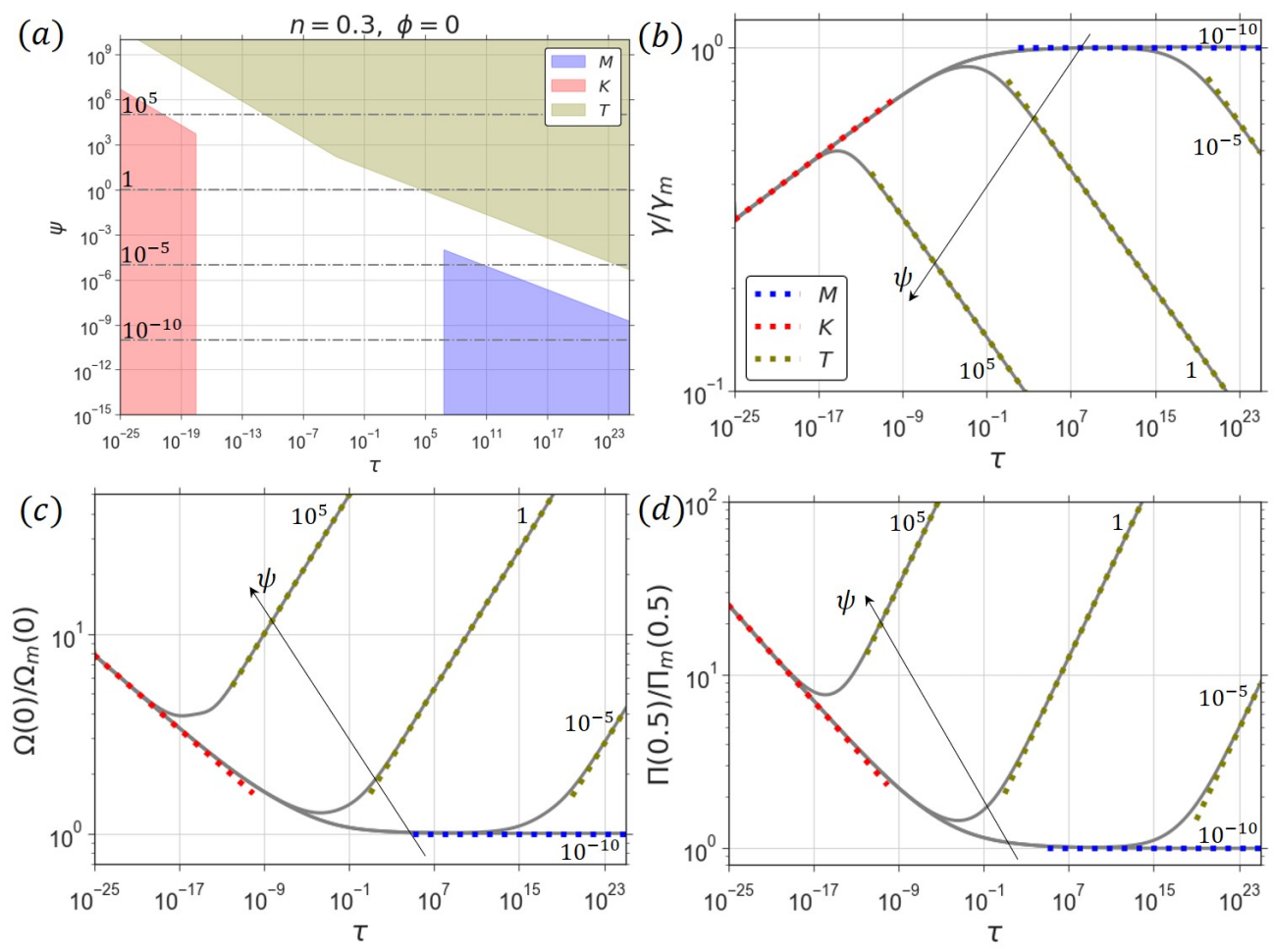

Figure 8: Results for a radial hydraulic fracture driven by the fluid with the flow behaviour index $n=0.3$ and non-zero yield stress in an impermeable rock $(\phi=0)$. The regime map $(\mathrm{a})$ is presented in the coordinates $(\tau, \psi)$, and the coloured regions denote the applicability domains of the limiting regimes. The time-dependent crack characteristics (radius (b), opening at the wellbore (c), and pressure at the half-radius (d)) normalised by the storage-viscosity limiting solution ( $M$ ) are depicted for the yield stress number values: $\psi=\left\{10^{-10}, 10^{-5}, 1,10^{5}\right\}$. In figure (a), the grey dash-dotted lines highlight the considered solution trajectories, while the coloured dotted lines in (b) - (d) mean the limiting solutions.

$\psi=\left\{10^{-10}, 10^{-5}, 1,10^{5}\right\}$ and construct the regime maps in the coordinates $(\tau, \phi)$. Then, we investigate variations of the major time-dependent crack properties for $\psi=1$ and different values of $\phi$.

Bingham fluid $(n=1)$

Figure 9 shows regime maps for a radial crack driven by fluid with $n=1$ and various values of yield stress. When the flow behaviour index $n>0.5$, the problem solution evolves from the storage-viscosity $(M)$ to the leak-off-toughness $(\tilde{K})$ regime for the power-law fluid $(\psi=0)$ or to the leak-off-yield-stress $(\tilde{T})$ vertex for the fluid with yield stress $(\psi>0)$. The applicability domains of the limiting propagation regimes for $\psi>0$ are shown by various colours in Figure 9, while the boundaries of the vertices for $\psi=0$ are depicted by coloured dashed lines. When the yield stress is absent, the storage-toughness $(K)$ and the leak-off-viscosity $(\tilde{M})$ regimes are realised as the intermediate asymptotes, and for $n=1$, they emerge along the intervals $\phi \lesssim 10^{-15}$ and $\phi \gtrsim 10^{3}$, respectively (see the coloured dashed lines in Figure 9). For a non-zero yield stress, the yield stress dominated regimes $T, \tilde{T}$ emerge earlier in the solution with an increase of $\psi$ (see the shifting of corresponding domains from Figure 9(b) to (d)). As a result, the validity zones of the toughness dominated regimes $K, \tilde{K}$ shrink with growing $\psi$, and eventually, they disappear completely (see Figure 9(a) versus (b) - (d)). Moreover, the domains belonging to the viscosity dominated regimes $M, \tilde{M}$ recede after a certain value of the dimensionless yield stress (see Figures 9(c), (d) as compared to (a), (b)); however, they always exist in the model for certain values of $\tau$ and $\phi$, and for all $\psi$. To summarise, during the intermediate time intervals, the general solution is approximated by $K, \tilde{K}, \tilde{M}, T$ vertex solutions depending on the values of the governing parameters $\phi$ and $\psi$. The boundaries between $K T, \tilde{K} \tilde{T}, T \tilde{T}$ transitions do not depend on the flow behaviour index in the range $n>0.5$ since this parameter affects only the regions of the parameter space where the dissipation effects related to the viscous fluid flow are significant, i.e., viscosity-toughness and viscosity-yield-stress transitions. Further, we compared the applicability regions of the limiting regimes for $n=1$ and $n=0.75$ (not shown) and found that the reduction of 

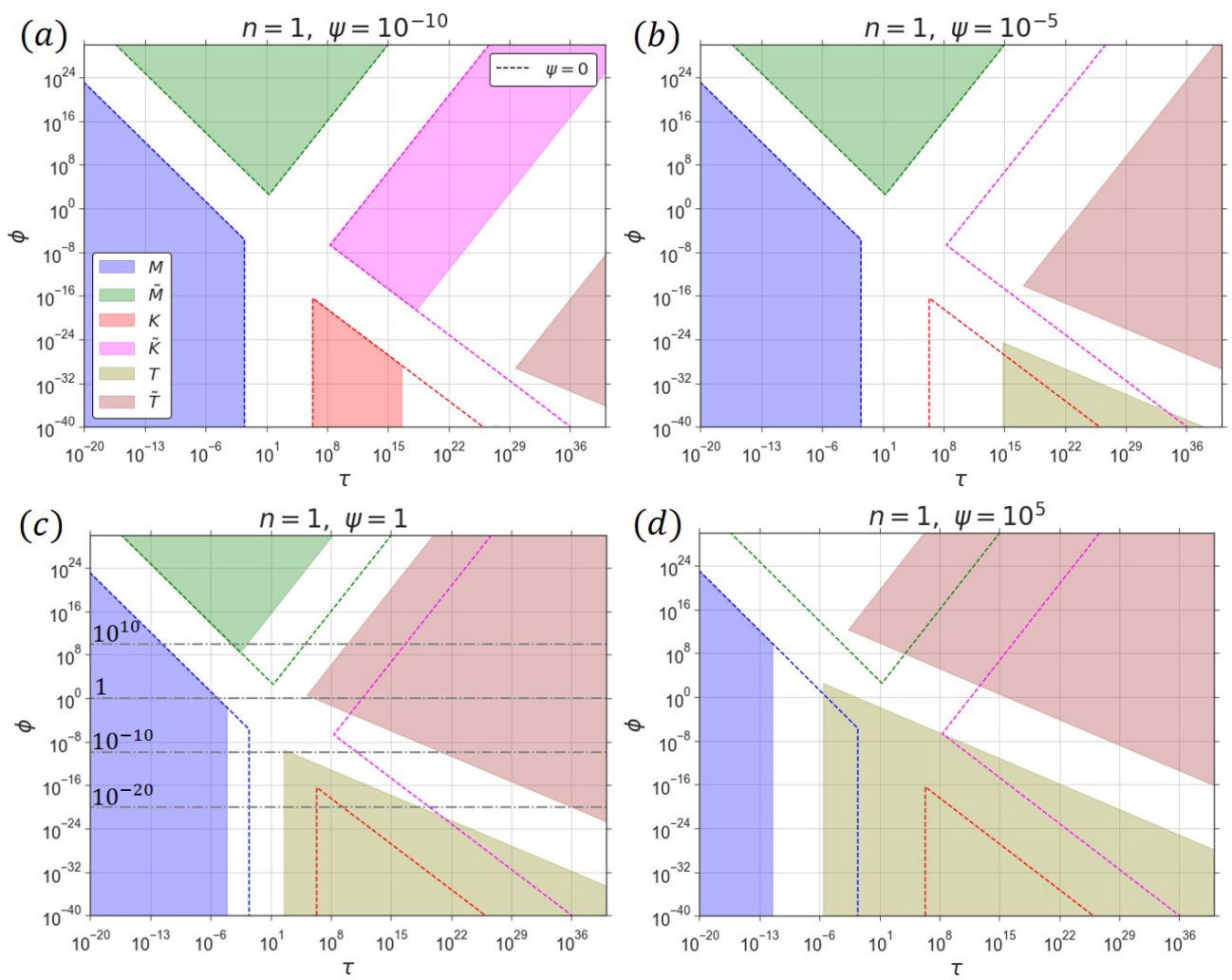

Figure 9: The parameter space for the problem of a radial hydraulic fracture driven by Bingham fluid ( $n=1)$ in a permeable rock $(\phi>0)$. The regime maps are depicted in the coordinates $(\tau, \phi)$ for several values of the dimensionless yield stress number: $\psi=\left\{10^{-10}, 10^{-5}, 1,10^{5}\right\}$. The applicability domains of the limiting propagation regimes are indicated by different colours, and the boundaries corresponding to $\psi=0$, i.e., Newtonian fluid case, are presented by the coloured dashed lines. In figure (c), the grey dash-dotted lines highlight the considered solution trajectories discussed in the current section.

the flow behaviour index within the interval $n \in(0.5,1]$ leads to further separation of $M, \tilde{M}, K, \tilde{K}$ regimes from the "center" of the map, i.e., the boundaries of $M$ and $K$ vertices framing the $M K$ transition move to the left (i.e., smaller times) and to the right (i.e., larger times), respectively, while the boundaries of $\tilde{M}$ and $\tilde{K}$ regimes framing the $\tilde{M} \tilde{K}$ transition shift up-left and down-right, correspondingly. The value of $n$ starts to influence locations of the boundaries of $T$ and $\tilde{T}$ regimes related to $M T$ and $\tilde{M} \tilde{T}$ transitions only for large values of $\psi$.

Further, we take a closer look at variations of the time-dependent properties of the radial crack driven by Bingham fluid $(n=1)$ with respect to parameters $\phi$ and $\psi$. (At this point, we should note that the following analyses are qualitatively applicable to the other values of the flow behaviour index inside the interval $0.5<n \leq 1$.) We consider the following set of the dimensionless leak-off values $\phi=\left\{10^{-20}, 10^{-10}, 1,10^{10}\right\}$ and perform computations for both zero (Newtonian fluid) and non-zero (Bingham fluid) yield stress $(\psi=1)$. The obtained results are demonstrated in Figure 10. The solid black lines show the solution with $\psi=1$, while the case $\psi=0$ is presented by the dashed grey lines. First, we discuss the dependence of the solution on the leak-off number $\phi$ for a fixed value of the yield stress number ( $\psi=0$ or $\psi=1$ ). We observe that the solution is independent of $\phi$ (and thus given by the impermeable case, $\phi=0$ ) during an initial time period of fracture propagation which duration depends on $\phi$ and $\psi$ values. Outside this initial time span, the increase of leak-off leads to smaller radius, opening at the wellbore, and crack efficiency, whereas the pressure becomes larger (Figure 10). However, the pressure behaviour can differ from the description provided above for the non-zero yield stress case in which pressure for all $\phi$ values tends to reach the same yield stress dominated asymptote (see the cyan line in Figure 10(c)).

Next, we focus on the effect of the yield stress by comparing the solutions for Newtonian $(\psi=0)$ and Bingham $(\phi=1)$ fluids at a fixed value of the leak-off number (Figure 10). These solutions are insensitive to the fluid yield 

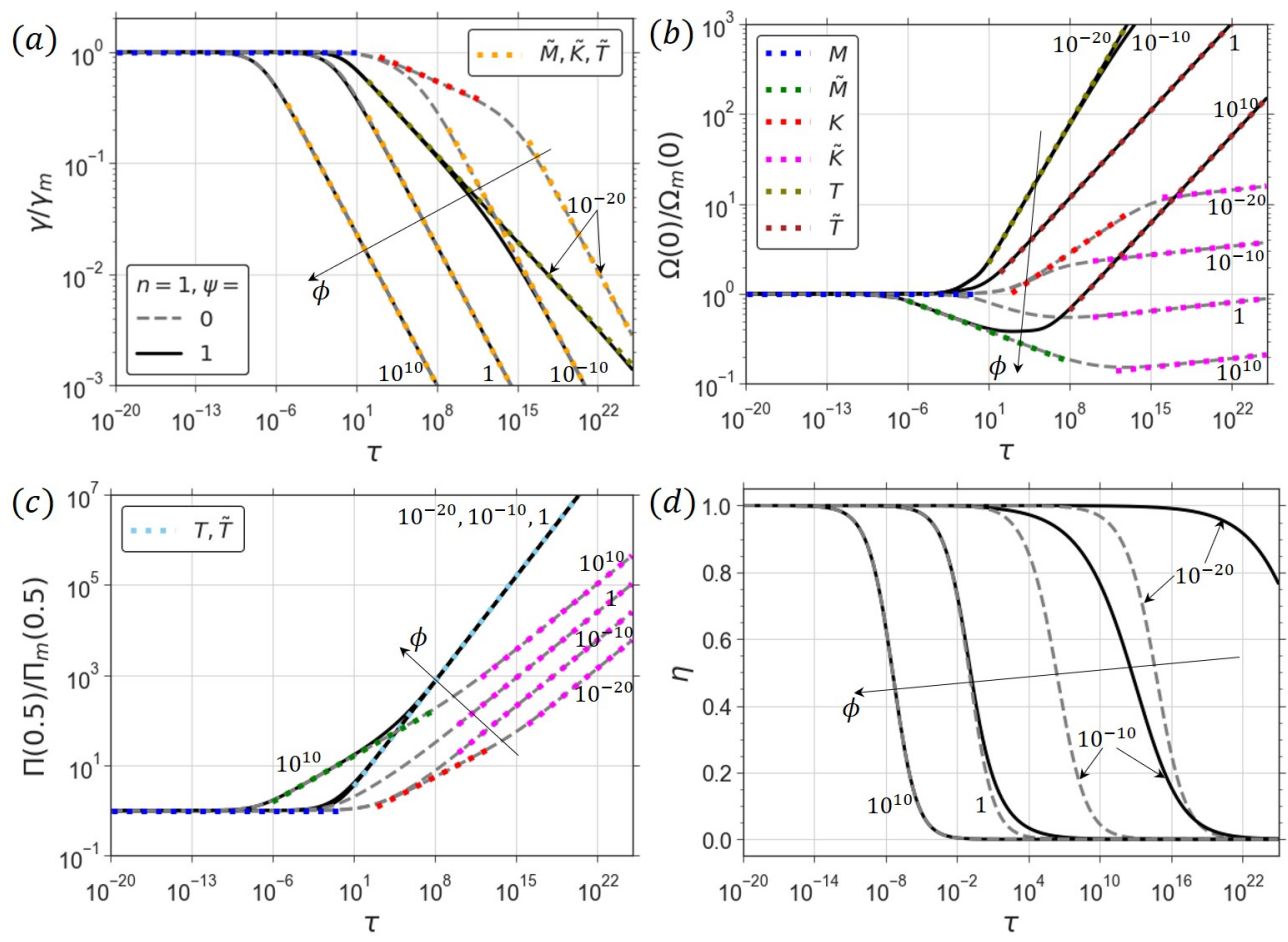

Figure 10: The time-dependent characteristics of a radial crack propagating in a permeable rock $(\phi>0)$ due to the injection of Bingham fluid: (a) radius $\gamma(\tau)$, (b) opening at the wellbore $\Omega(0, \tau)$, (c) pressure at the half-radius $\Pi(1 / 2, \tau)$, and $(\mathrm{d})$ efficiency $\eta(\tau)$. The properties in charts $(\mathrm{a})-(\mathrm{c})$ are normalised by the storage-viscosity $(M)$ limiting solution. The profiles corresponding to $\phi=\left\{10^{-20}, 10^{-10}, 1,10^{10}\right\}$ and $\psi=1$ are shown by the solid black lines. The solutions for the same values of leak-off and zero yield stress are depicted by the dashed grey lines for comparison. The vertex solutions are presented by the dashed coloured lines. In graph (a), the asymptotic behaviour of the radius in the leak-off dominated regimes $\tilde{K}, \tilde{M}, \tilde{T}$ are represented by the orange colour due to the same functional relationships $\gamma_{\tilde{K}}(\tau), \gamma_{\tilde{M}}(\tau), \gamma_{\tilde{T}}(\tau)$ for all of them. Similarly, in graph (c), we apply cyan colour for the pressure behaviour in the the yield stress dominated regimes $T, \tilde{T}\left(\Pi_{T}(1 / 2, \tau), \Pi_{\tilde{T}}(1 / 2, \tau)\right)$ which are very close to each other.

stress and thus coincide at the early stages of crack growth. Moreover, when the leak-off is very large $\left(\phi=10^{10}\right)$, two solutions match in the entire time domain for the radius and efficiency (Figures 10(a), (d)). At larger time the fluid yield stress $(\psi=1)$ leads to the reduced value of the radius and increased opening at the wellbore, pressure, and efficiency compared to the Newtonian fluid case $(\phi=0)$.

The time domains where the fracture propagation is dominated by different limiting regimes can be identified in Figure 10 by comparing the computed fracture properties with the vertices (Appendix B) shown by the coloured dashed lines. We note that the limiting solutions for the radius are identical in all leak-off dominated regimes and thus shown in Figure 10(a) by a single (orange) colour. Similarly, the limiting solutions for pressure is all yield stress dominated regimes in Figure 10(c) are depicted by same colour (cyan) line.

Herschel-Bulkley fluid with $n=0.3$

We now turn to the discussion of the parametric dependence of the solution for a radial crack propagation in a permeable rock $(\phi>0)$ due to the injection of Herschel-Bulkley fluid with the flow behaviour index $n=0.3$. Figure 11 shows the computed propagation regime maps. When the flow behaviour index $n<0.5$, the problem solution starts at the storage-toughness $(K)$ regime and finishes at the leak-off-viscosity $(\tilde{M})$ regime for the power-law fluid $(\psi=0)$ or at the leak-off-yield-stress $(\tilde{T})$ regime for Herschel-Bulkley fluid $(\psi>0)$. The validity zones of the limiting regimes corresponding to $\psi>0$ cases are shown by various colours in Figure 9, where we also plot the boundaries of the corresponding zones for the power-law fluid case $\psi=0$ by dashed lines for comparison purposes. The general solution may approach the leak-off-toughness $(\tilde{K})$, storage-viscosity $(M)$, leak-off-viscosity $(\tilde{M})$, and/or storage-yield-stress $(T)$ regimes at an intermediate time depending on the values of $\phi$ and $\psi$. The behaviour of the yield 

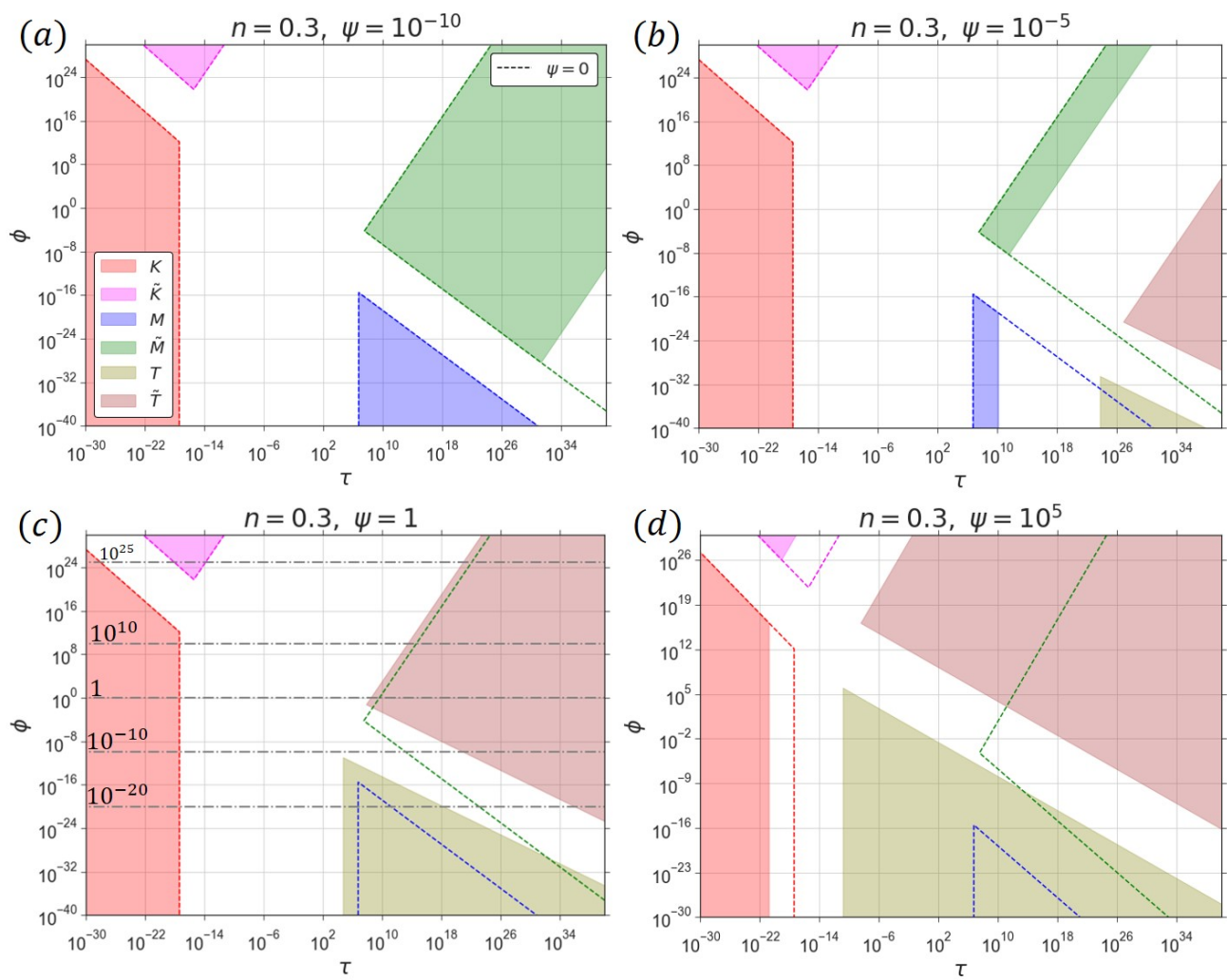

Figure 11: The parameter space of the model for a radial hydraulic fracture driven by Herschel-Bulkley fluid with $n=0.3$ in a permeable rock $(\phi>0)$. The regime maps are depicted in the coordinates $(\tau, \phi)$ for several values of the dimensionless yield stress: $\psi=\left\{10^{-10}, 10^{-5}, 1,10^{5}\right\}$. The applicability domains of the limiting propagation regimes are filled by different colours, and the boundaries corresponding to $\psi=0$, i.e., power-law fluid, are presented by coloured dashed lines. In figure (c), the grey dash-dotted lines highlight the considered solution trajectories discussed in the current section.

stress dominated regimes with an increase of $\psi$ is qualitatively similar to the already discussed case of the Bingham fluid, namely, $T$ and $\tilde{T}$ domains expand towards smaller times. This shift leads to shrinking and, eventually, vanishing time domains of the viscosity dominated regimes as it can be noticed for $n=0.3$ from Figures 11(a), (b) as compared to the panels (c), (d), and to the shrinking of the toughness regimes time domains (see Figure 11(d)). In contrast to $n>0.5$ case, for $n<0.5$ the change of the dimensionless yield stress does not affect the boundaries framing the $T \tilde{T}$ transition. We also should comment on the alterations of the regime map with the variation of the flow behaviour index inside the interval $n \in[0,0.5$ ) (not shown). When we increase the value of $n$, the boundaries of $K$ and $M$ vertex solutions framing the $K M$ transition move to the left (i.e., smaller times) and to the right (i.e., larger times) respectively, whereas the boundaries of $\tilde{K}$ and $\tilde{M}$ domains framing the $\tilde{K} \tilde{M}$ transition go up-left and down-right, correspondingly. Similarly to $n>0.5$, the flow behaviour index affect the boundaries of the yield stress dominated regimes framing $K T$ and $\tilde{K} \tilde{T}$ transitions only for the large values of the dimensionless yield stress.

Figure 12 illustrates the evolution of fracture characteristics with time for the Herschel-Bulkley fluid $(n=0.3$, $\psi=1$ ) for various values of the leak-off number: $\phi=\left\{10^{-20}, 10^{-10}, 1,10^{10}, 10^{25}\right\}$ (see the corresponding dashdotted trajectories in the parametric map in Figure 11(c)). The solutions for the power-law fluid case $(n=0.3, \psi=0)$ are also shown for comparison by grey dashed lines. (Note that the solutions for the radius, maximum opening, and pressure at the half-radius in panels (a)-(c) in Figure 12 are normalised by the storage-viscosity limiting solution $(\boldsymbol{M})$.) One can notice that the fluid yield stress impacts the problem solution qualitatively similar to the Bingham fluid case: (i) for the fixed yield stress number, the increase of leak-off results in the fracture with smaller radius, maximum opening, and efficiency, but higher fluid pressure; (ii) when we fix the leak-off number and raise the yield stress number, we observe the reduction of the radius and increase of opening at the wellbore, pressure, and efficiency. The tendencies (i) and (ii) are applicable for the large enough time when the solutions for Herschel-Bulkley fluid $(\psi>0)$ and Newtonian 

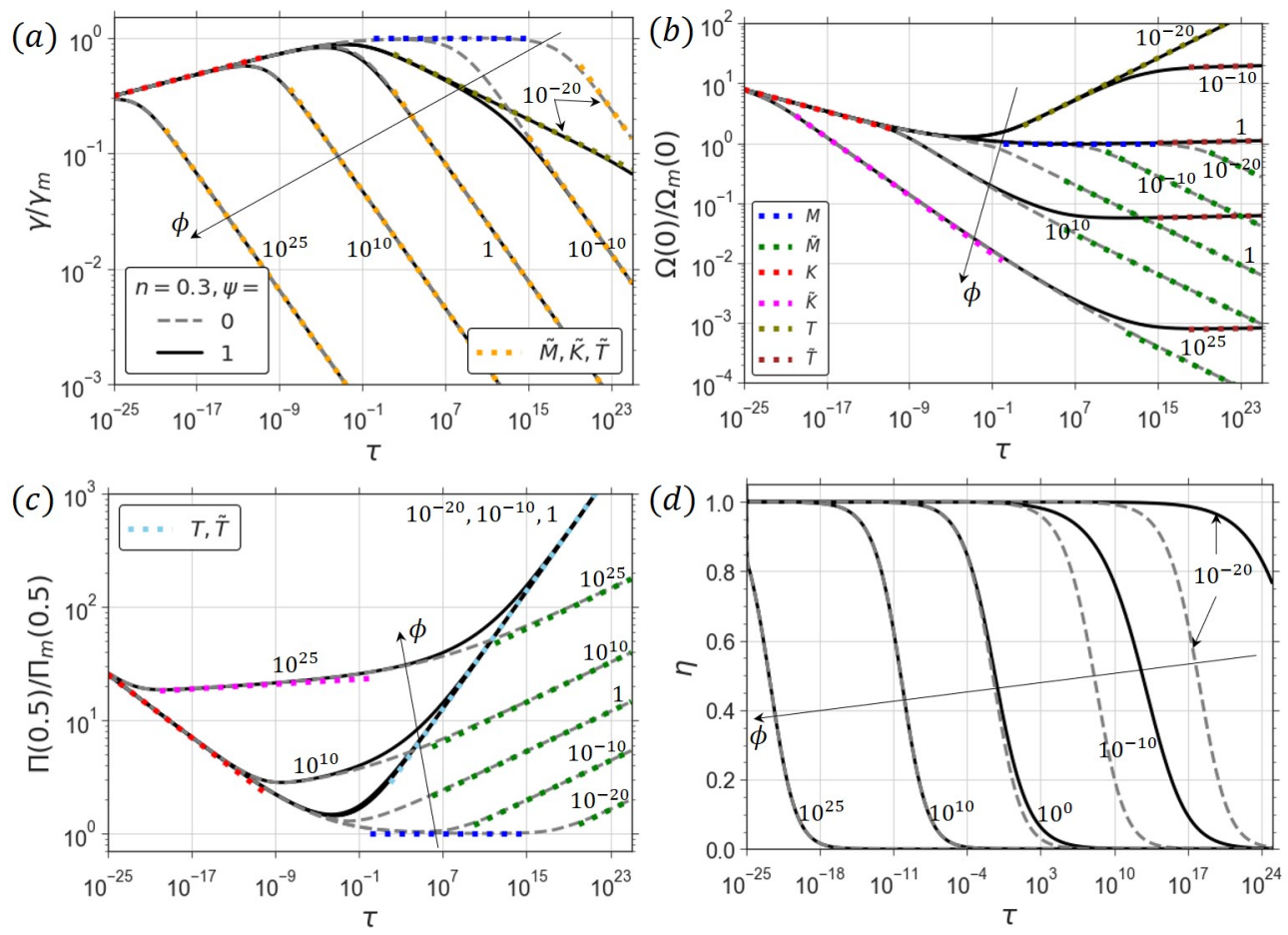

Figure 12: The time-dependent characteristics of a radial crack propagating in a permeable rock $(\phi>0)$ due to the injection of Herschel-Bulkley fluid with $n=0.3$ : (a) radius $\gamma(\tau)$, (b) maximum opening $\Omega(0, \tau)$, (c) pressure at the halfradius $\Pi(1 / 2, \tau)$, and $(\mathrm{d})$ efficiency $\eta(\tau)$. The properties in charts (a) - (c) are normalised by the storage-viscosity $(M)$ limiting solution. The profiles corresponding to $\phi=\left\{10^{-20}, 10^{-10}, 1,10^{10}, 10^{25}\right\}$ and $\psi=1$ are shown by the solid black lines. The solutions for the same values of leak-off and zero yield stress case are depicted by the dashed grey lines for comparison. The vertex solutions are presented by the dashed coloured lines. In graph (a), the asymptotic behaviour of radius in the leak-off dominated regimes $\tilde{K}, \tilde{M}, \tilde{T}$ are represented by the orange colour due to the same functional relationships $\gamma_{\tilde{K}}(\tau), \gamma_{\tilde{M}}(\tau), \gamma_{\tilde{T}}(\tau)$ for all of them. Similarly, in graph (c), we apply the cyan colour for the pressure behaviour in the yield stress dominated regimes $T, \tilde{T}\left(\Pi_{T}(1 / 2, \tau), \Pi_{\tilde{T}}(1 / 2, \tau)\right)$ which are very close to each other.

fluid $(\psi=0)$ are distinct. For a better perception of the crack propagation regimes realised at different time in the demonstrated solutions, we plot the limiting (vertex) solutions (Appendix B) by the dashed coloured lines in Figure 12.

\subsection{Quantitative estimations of the plug zone}

In the current model for the hydraulic fracture driven by Herschel-Bulkley fluid, the plug zone is formed inside the regions where the shear stress $\tau_{m}$ is less than the yield stress $\tau_{0}$, and its width is equal to $w_{\text {plug }}(\rho, t)=2 y_{\tau}=$ $2 \tau_{0} R|\partial p / \partial \rho|^{-1}$. To track evolution of the unyielded zone, we introduce the time-dependent characteristic $\Upsilon(t)$ defined as volumetric fraction of the plug within the crack, i.e., $\Upsilon(t)=V_{\text {plug }}(t) / V_{\text {crack }}(t)=\int_{0}^{1} \rho w_{\text {plug }} d \rho /\left[\int_{0}^{1} \rho w d \rho\right]$. Further, we express $\Upsilon(t)$ through the dimensionless crack properties (57) and the governing parameters $\phi, \psi(56)$ as:

$$
\Upsilon(\tau, \phi, \psi)=2 \psi \gamma \frac{\int_{0}^{1} \rho\left|\frac{\partial \Pi}{\partial \rho}\right|^{-1} d \rho}{\int_{0}^{1} \rho \Omega d \rho} .
$$

Equation (60) can be also simplified for the impermeable rock case as follows:

$$
\Upsilon(\tau, 0, \psi)=\frac{4 \pi \psi \gamma^{3}}{\tau} \int_{0}^{1} \rho\left|\frac{\partial \Pi}{\partial \rho}\right|^{-1} d \rho
$$



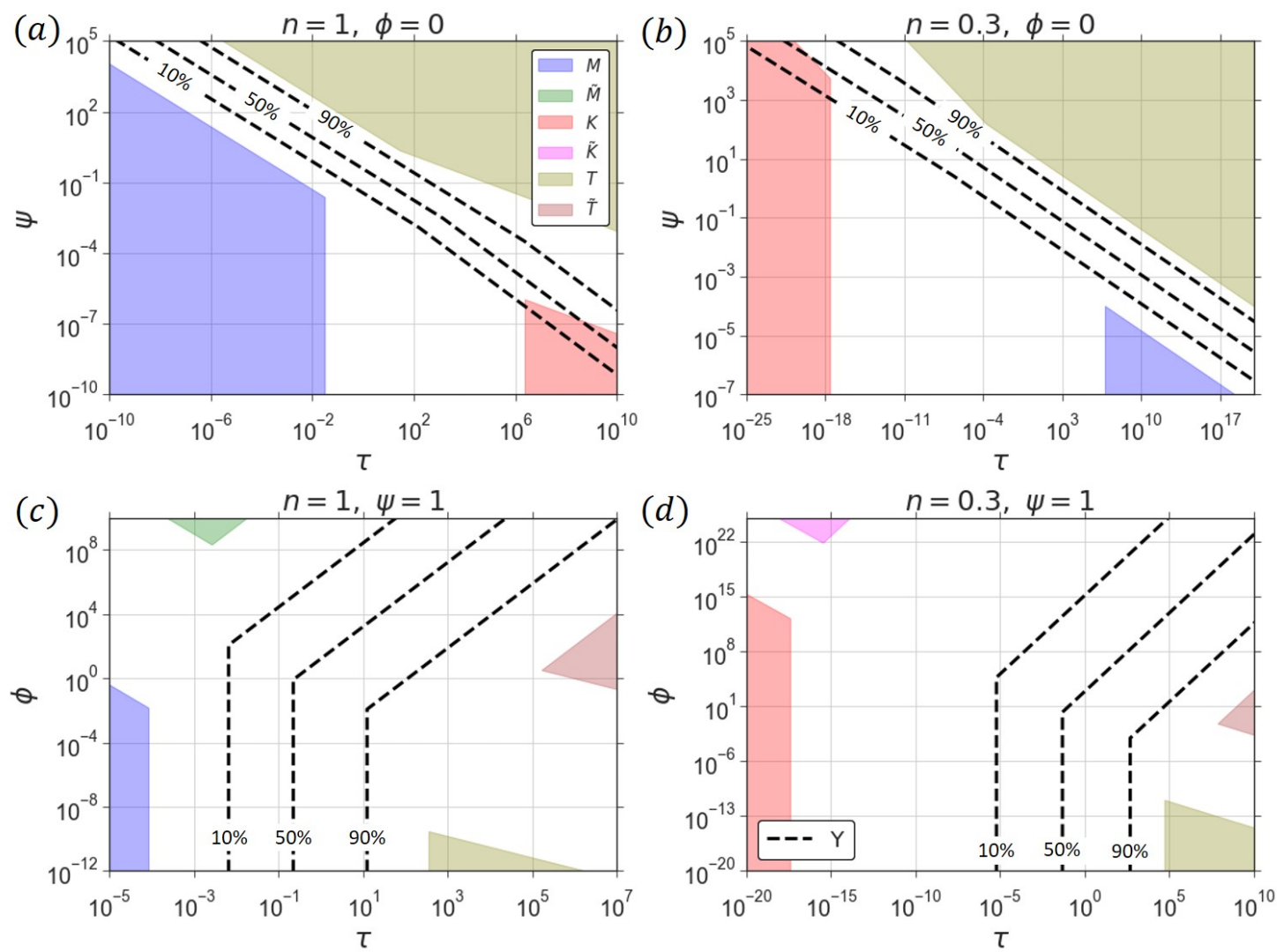

Figure 13: The charts present the isolines for $\Upsilon(\tau, \phi, \psi)=V_{\text {plug }} / V_{\text {crack }}$ (the dashed black lines) corresponding to the quantities $10 \%, 50 \%$, and $90 \%$. Two cases of the flow behaviour index are analysed: $n=1$ (panels (a) and (c)) and $n=0.3$ (panels (b) and (d)). The top row reflects the impermeable reservoir case, while the bottom figures correspond to simulations with leak-off and for the yield stress number $\psi=1$. The regime maps demonstrated in Figures 7, 8, 9, 11 are applied as background in the diagrams.

The fully numerical solution (Section 3.1) is utilised for calculating $\Upsilon(\tau, \phi, \psi)$ time histories for various $\phi, \psi$ since the approximate approach (Section 3.2) provides the pressure gradient profiles with a reduced accuracy. The results are shown in Figure 13 in the form of the isolines (the dashed black lines) $\Upsilon(\tau, \phi, \psi)=$ const where the constant can be varied within the segment $[0,1]$ and, in our case, takes values $0.1,0.5$, and 0.9 . The plug fraction isolines are shown for two values of the flow behaviour index $n=1$ (the left column) and $n=0.3$ (the right column) for both impermeable (the top row) and permeable (the bottom row) formation cases. We demonstrate the results in the parameter space $(\tau, \psi)$ when $\phi=0$ and in the space $(\tau, \phi)$ for the fixed yield stress of $\psi=1$ for non-zero leak-off $\phi>0$. To better understand positions of the isolines relatively to the limiting regimes, we add their validity zones using the same colour palette as in the regime maps in Figures 7, 8, 9, 11.

The selected isolines $\Upsilon(\tau, \phi, \psi)=$ const are predominantly located in the transition regions between the limiting propagation regimes. For a crack driven by Bingham fluid in an impermeable rock (panel (a) in Figure 13), $M T$ and $K T$ transitions contain the contour lines within the time intervals $\tau \lesssim 10^{-2}$ and $\tau \gtrsim 10^{6}$, respectively. The reversed situation is observed for the Herschel-Bulkley fluid with $n=0.3$ (panel (b) in Figure 13) in which the isolines are located inside the $K T$ and $M T$ transition zones for $\tau \lesssim 10^{-18}$ and $\tau \gtrsim 10^{7}$, correspondingly. The isoline $\Upsilon=0.1$ passes closely to the applicability domains of the storage limiting regimes, i.e., viscosity $M$ and toughness $K$, in which $\Upsilon=0$. In turn, the level $\Upsilon=0.9$ lies near the validity region of the $T$ vertex associated with $\Upsilon=1$.

Generally, the function $\Upsilon(\tau, \phi, \psi)$ for the fixed values of the governing parameters $\phi$ and $\psi$ grows smoothly with time from 0 to 1 evolving from the viscosity $M, \tilde{M}$ or toughness $K, \tilde{K}$ limiting regimes to the yield-stress $T, \tilde{T}$ vertices. In the model with the non-zero leak-off (panels (c) and (d) in Figure 13), we observe that the isolines belong to $M T$ 
$(K T)$ and $\tilde{M} \tilde{T}(\tilde{K} \tilde{T})$ transitions when the leak-off number $\phi \ll 1$ and $\phi \gg 1$, respectively, and $n=1(n=0.3)$. Moreover, one can notice that the contour lines are parallel to the boundaries of the applicability domains of the vertex solutions framing the transition zones enclosing the isolines.

Finally, we focus on the behaviour of the isolines near the toughness dominated limiting regimes when the leak-off number $\phi$ is fixed, and the yield stress number $\psi$ is small (for $n>0.5$ ) or large (for $n<0.5$ ). Using the panels (a) and (b) in Figure 13 for $\phi=0$ cases, it is possible to observe that the contour lines for $\Upsilon=0.1,0.5$ penetrate into the validity zone of the storage-toughness regime. This phenomenon can be explained in the following way: the unyielded region starts to form when the crack geometry (radius and aperture) corresponds to the $K$ vertex solution in which the energy is spent primarily on the brittle rock failure and is independent of the fluid flow process inside the crack channel where the coexistence of both liquid and solid (plug) states can occur.

\subsection{Simulations of the crack growth for typical field cases}

This subsection outlines results of the radial crack propagation in terms of the dimensional variables (the system of the governing equations can be found in Section 2.2). We choose the values of the input parameters representative of typical field applications, and they consist of the geomechanical and filtration-storage properties of the porous rock, fluids (pore and hydraulic fracturing) characteristics, and the injection rate. The main aim of the analysis is to examine the impact of non-zero yield stress on the problem solution quantitatively for particular cases relevant to the field.

We consider fracture propagation during the first $6 \cdot 10^{3}$ seconds of injection with the volumetric rate of $Q_{0}=$ $0.01 \mathrm{~m}^{3} / \mathrm{s}$. The set of the geomechanical parameters is: plane-strain elastic modulus $E^{\prime}=30 \mathrm{GPa}$, rock toughness $K_{I c}=1 \mathrm{MPa} \cdot \sqrt{\mathrm{m}}$, far-field confining stress $\sigma_{o}=10 \mathrm{MPa}$. We take the fracturing fluid with the flow behaviour index $n=0.7$, consistency index $M=1 \mathrm{~Pa} \cdot \mathrm{s}^{\mathrm{n}}$ providing $M^{\prime}=7.7 \mathrm{~Pa} \cdot \mathrm{s}^{\mathrm{n}}$, and the yield stress value is $\tau_{0}=$ $10 \mathrm{~Pa}$ (since we estimate the yield stress influence, the reference solution corresponds to the power-law fluid with the same $n$ and $M$ but $\tau_{0}=0$ ). We analyse the crack propagation in both impermeable and permeable formations. In the latter case, we consider the pore fluid and formation characteristics to be the following: far-field pore pressure $p_{o}=6 \mathrm{MPa}$, permeability $k=10 \mathrm{mD}$, porosity $\phi_{r}=20 \%$, viscosity $\mu=5 \mathrm{cP}$ (Newtonian fluid), compressibility $c_{t}=10^{-4} \mathrm{~atm}^{-1}$. For simplicity, it is assumed that the filtrate of the fracturing fluid has the same properties as the pore fluid. Consequently, the leak-off parameter is equal to $C^{\prime}=9.1 \cdot 10^{-5} \mathrm{~m} / \sqrt{\mathrm{s}}$ in the permeable reservoir case. In terms of the dimensionless governing parameters $(\tau, \phi, \psi)$ introduced in Section 4.2, the analysed cases can be written as: $\tau \in\left(0,1.04 \cdot 10^{-10}\right), \phi=0$ (impermeable rock) or $\phi=4.7 \cdot 10^{14}$ (permeable rock), $\psi=5.8 \cdot 10^{4}$ (Herschel-Bulkley fluid) or $\psi=0$ (power-law fluid).

Figure 14 shows evolution in time of the fracture radius $R(t)$, opening at the wellbore $w(0, t)$, net pressure at the half-radius $p(R(t) / 2, t)$, and efficiency $\eta(t)$ calculated using fully numerical solution (see Section 3.1). The results for a radial crack driven by the Herschel-Bulkley fluid are depicted by the solid lines, for a crack driven by power-law fluid by the dashed lines. We utilise the blue colour for the zero leak-off case and the green one for when the rock formation is permeable.

We observe that the non-zero yield stress $\tau_{0}=10 \mathrm{~Pa}$ leads to the reduction of the crack radius, increase of the opening at the wellbore, pressure, and efficiency (there is no alteration in efficiency for the impermeable formation case) (see the solid lines versus the dashed ones of the same colour in Figure 14). Therefore, these findings are in the agreement with the analyses stated in Section 5.2. To characterise the discrepancies between two compared solutions, i.e., for Herschel-Bulkley ("hb") and power-law ("pl") fluids, quantitatively, we calculate the relative differences between the crack properties for the time moment $t=6000 \mathrm{~s}$ using the following formula: $\delta_{A}=\left|A_{\mathrm{hb}}-A_{\mathrm{pl}}\right| / A_{\mathrm{hb}}$, where $A$ is an analysed parameter. The obtained metrics are summarised in Table 2. One can notice that the variations between the two compared solutions are more substantial during the fracture propagation in an impermeable rock. Here, $\delta_{A}$ for the radius and maximum aperture parameters is approximately $9 \%$ and $20 \%$, correspondingly. When leak-off is introduced into the model, the radius values are almost indistinguishable, while the opening at the wellbore and crack efficiency are higher for Herschel-Bulkley fluid by $11.4 \%$ and $8.4 \%$, respectively. Finally, $\delta_{A}$ for the net pressure at the half-radius reaches approximately $27 \%$ and $13 \%$ for $\phi=0$ and $\phi>0$. All these clearly demonstrate the importance of yield stress for modelling practical cases of hydraulic fracturing.

\section{Conclusions}

In this paper, we constructed a numerical model for a radial hydraulic fracture driven by Herschel-Bulkley fluid in a permeable rock and carried out extensive analysis of the combined impact of the fluid yield stress and leak-off on the 

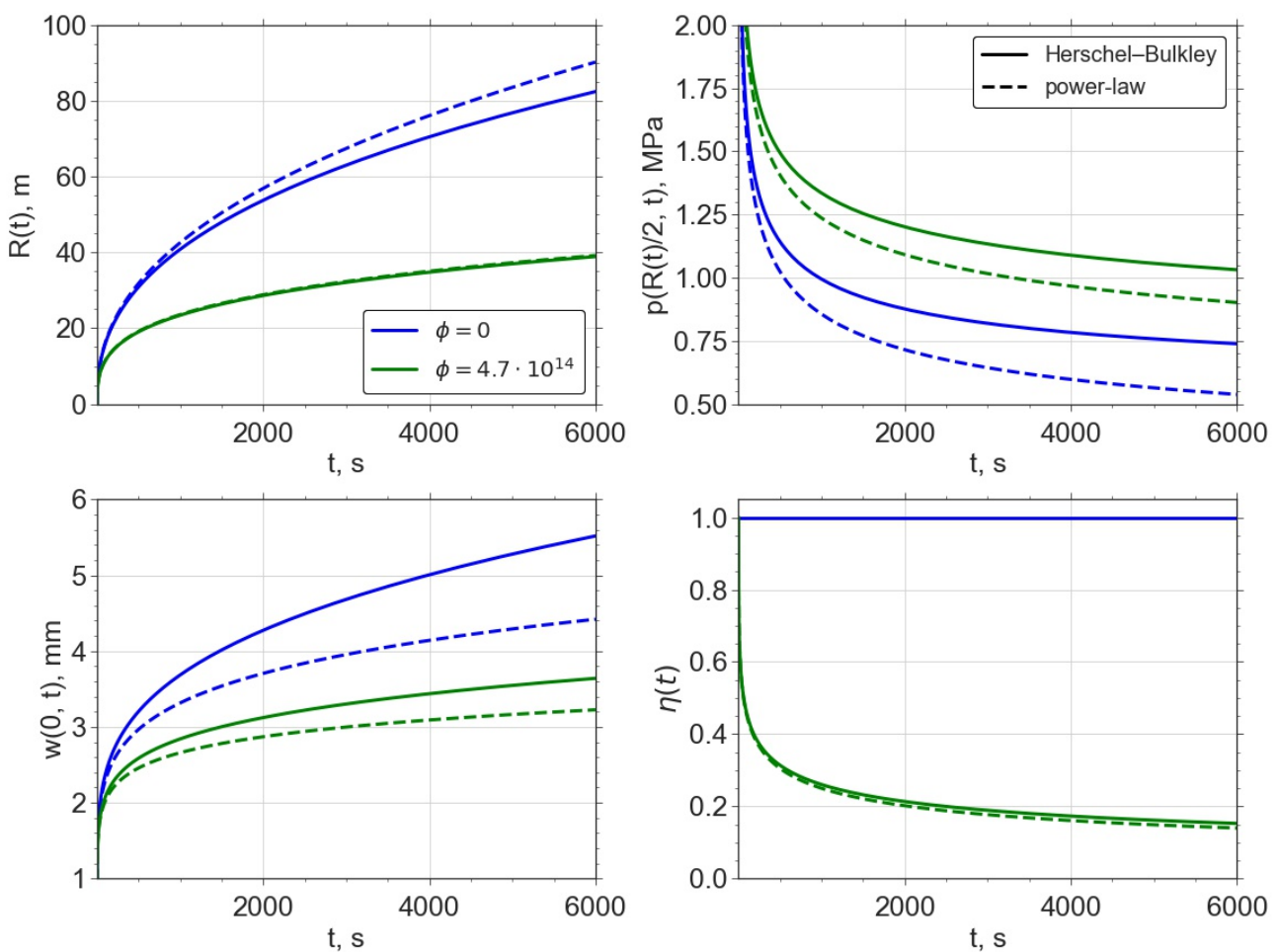

Figure 14: The figure illustrates various time-dependent radial crack parameters computed using fully numerical solution (Section 3.1). The left column shows the radius $R(t)$ and aperture near the wellbore $w(0, t)$ evolutions, while the right column shows the net pressure $p(R(t) / 2, t)$ and the efficiency $\eta(t)$ variations. The solid lines correspond to the cases of the crack driven by the Herschel-Bulkley fluid, and the dashed lines are utilised for the solutions corresponding to the fluid with power-law rheology. The blue and green colours denote the impermeable and permeable formation cases, respectively.

\section{Table 2}

The relative differences $\left(\delta_{A}\right)$ between various radial fracture parameters $(\mathrm{A})$ corresponding to the fracturing fluids with the Herschel-Bulkley ("hb") and power-law ("pl") rheologies that are calculated at $t=6000 \mathrm{~s}$.

\begin{tabular}{|c|c|c|}
\hline \multicolumn{3}{|c|}{$\delta_{A}=\left|A_{\mathrm{hb}}-A_{\mathrm{pl}}\right| / A_{\mathrm{hb}}$ (in \%) } \\
\hline Parameter $(\mathrm{A})$ & $\phi=0$ & $\phi=5.8 \cdot 10^{4}$ \\
\hline$R(t)$ & 9.4 & 0.8 \\
\hline$w(0, t)$ & 19.9 & 11.4 \\
\hline$p(R(t) / 2, t)$ & 27.2 & 12.6 \\
\hline$\eta(t)$ & 0 & 8.4 \\
\hline
\end{tabular}

crack propagation. In most of the existing models, the fracturing fluid is taken as Newtonian or power-law; however, certain fracturing fluids, such as gels and foams, demonstrate shear-thinning rheology with the yield stress that can be described by the Herschel-Bulkley model. The system of governing equations is formulated in the dimensional and normalised forms, and, for the latter, all crack characteristics are governed by two dimensionless parameters: the leak-off number $\phi$ and the yield stress number $\psi$. Two different approaches are used to obtain the problem solution: the accurate method based on Gauss-Chebyshev quadrature and Barycentric Lagrange interpolation techniques and the simplified algorithm that utilises the global fluid balance equation together with spatial continuation of the neartip asymptotic model. We examine the limiting propagation regimes characterised by certain energy dissipation and fluid balance mechanisms. Further, we investigate the problem parametric space using the dimensionless formulation by looking at the validity domains of the vertices and variations of different time-dependent crack characteristics versus the dimensionless problem parameters. The regime maps allow us to rapidly detect the dominance of various physical processes in a hydraulic fracture for certain values of the governing parameters without running numerical 
simulations. Using the obtained results, we conclude that the non-zero yield stress leads to a radial crack with a smaller radius, larger crack opening at the wellbore, pressure, and efficiency (for a permeable rock case) as compared to the power-law fluid case. Next, we simulate the radial fracture growth by taking the input parameters close to typical field applications and reveal that the yield stress can potentially result in notable deviations of the fracture parameters from the outcomes of zero yield stress model. The proposed radial crack model is applicable not only for computation of fracture parameters for the radial hydraulic fracture driven by fluid with the non-zero yield stress, but also for validating numerical calculations of more complex numerical simulators, such as a three-dimensional planar fracture (Planar3D) model, in which the Herschel-Bulkley rheology is also employed.

\section{Acknowledgements}

This work was supported by the Ministry of Science and Higher Education of the Russian Federation under agreement no. 075-15-2020-119 within the framework of the development program for a world-class Research Center. Support by the Natural Science and Engineering Research Council of Canada, Discovery Program grant no. 05743, to D.I. Garagash is also gratefully acknowledged.

\section{Appendices}

\section{A. Calculation of $\lambda$ for the approximate numerical solution (Section 3.2)}

In Section 4.1, we outline the values of $\lambda$ for all limiting propagation regimes realised in the model: $\lambda_{m}=\lambda_{m}(n)$ (see Figure 2(a)), $\lambda_{\tilde{m}}=\lambda_{\tilde{m}}(n)$ (see Figure 4(a)), $\lambda_{k}=\lambda_{\tilde{k}}=0.5, \lambda_{t}=1.029$, and $\lambda_{\tilde{t}}=1.077$. As it was demonstrated in Section 5.2, the validity regions of the vertex solutions strongly depend on time and the governing parameters. Consequently, based on the values of $\lambda$ for the vertices, it is necessary to construct an interpolation scheme allowing one to determine $\lambda$ for all possible values of input parameters.

Let us define the auxiliary set of parameters:

$$
\Xi_{i}=w_{i}(0, t) / R_{i}(t), \quad \eta_{\mathrm{pl}}=\frac{2 \pi R^{2} w_{a}(R) \mathcal{B}(0.5, \bar{\delta})}{Q_{0} t}, \quad \eta_{\tau_{0}}=\frac{2 \pi R^{2} w_{a}(R) \mathcal{B}(1.05, \bar{\delta})}{Q_{0} t},
$$

where the subscript $i$ denotes the considered limiting regime, and it can take values $i=\{m, \tilde{m}, k, \tilde{k}, t, \tilde{t}\}$. The radius $R_{i}(t)$ and wellbore opening $w_{i}(0, t)$ evolution in the vertex solutions are defined by the equations (34), and the function $\mathcal{B}(\lambda, \bar{\delta})$ given by equation (27). The parameter $\eta_{\mathrm{pl}}$ can be interpreted as the approximate efficiency for the crack filled by the power-law fluid, and the similar interpretation can be done for $\eta_{\tau_{0}}$ which is more suitable for the yield-stress dominated regimes $T, \tilde{T}$.

Using the variables (61), we can construct the following interpolation function for $\lambda$ :

$$
\lambda=\frac{\lambda_{m}\left(\Xi_{m} \eta_{\mathrm{pl}}\right)^{\kappa}+\lambda_{k}\left(\Xi_{k} \eta_{\mathrm{pl}}\right)^{\kappa}+\lambda_{\tilde{m}}\left[\Xi_{\tilde{m}}\left(1-\eta_{\mathrm{pl}}\right)\right]^{\kappa}+\lambda_{\tilde{k}}\left[\Xi_{\tilde{k}}\left(1-\eta_{\mathrm{pl}}\right)\right]^{\kappa}+\lambda_{t}\left(\Xi_{t} \eta_{\tau_{0}}\right)^{\kappa}+\lambda_{\tilde{t}}\left[\Xi_{\tilde{t}}\left(1-\eta_{\tau_{0}}\right)\right]^{\kappa}}{\left(\Xi_{m} \eta_{\mathrm{pl}}\right)^{\kappa}+\left(\Xi_{k} \eta_{\mathrm{pl}}\right)^{\kappa}+\left[\Xi_{\tilde{m}}\left(1-\eta_{\mathrm{pl}}\right)\right]^{\kappa}+\left[\Xi_{\tilde{k}}\left(1-\eta_{\mathrm{pl}}\right)\right]^{\kappa}+\left(\Xi_{t} \eta_{\tau_{0}}\right)^{\kappa}+\left[\Xi_{\tilde{t}}\left(1-\eta_{\tau_{0}}\right)\right]^{\kappa}},
$$

where $\kappa$ is a fitting parameter which is the current study is set 3. We also should mention that for the zero leak-off case $\Xi_{\tilde{m}}=\Xi_{\tilde{k}}=\Xi_{\tilde{t}}=0$. Similarly, when we simulate the radial crack driven by the power-law fluid, we set $\Xi_{t}=\Xi_{\tilde{t}}=0$.

\section{B. Limiting propagation regimes (summary of Section 4.1)}

\section{$M$-vertex}

Dimensional form:

$$
\begin{aligned}
& R_{m}(t)=R_{m}^{*}\left(\frac{E^{\prime} Q_{0}^{n+2} t^{2 n+2}}{M^{\prime}}\right)^{1 /(3 n+6)}, \quad w_{m}(\rho, t)=w_{m}^{* *}\left(\frac{M^{\prime 2} Q_{0}^{n+2} t^{2-n}}{E^{\prime 2}}\right)^{1 /(3 n+6)}(1+\rho)^{\lambda_{m}}(1-\rho)^{\bar{\delta}_{m}}, \\
& p_{m}(\rho, t)=p_{m}^{*}\left(\frac{M^{\prime} E^{\prime n+1}}{t^{n}}\right)^{1 /(2+n)} \mathcal{F}\left(\rho, \lambda_{m}, \bar{\delta}_{m}\right) .
\end{aligned}
$$


Normalised form:

$$
\gamma_{m}(\tau)=R_{m}^{*} \tau^{(2 n+2) /(3 n+6)}, \quad \Omega_{m}(\rho, \tau)=w_{m}^{* *} \tau^{(2-n) /(3 n+6)}, \quad \Pi_{m}(\rho, \tau)=p_{m}^{*} \tau^{-n /(2+n)} .
$$

Parameters:

$$
\begin{aligned}
& R_{m}^{*}=\left(2 \pi \beta_{m} \alpha_{m}^{n /(n+2)} \mathcal{B}_{m}\right)^{-1 / 3}, \quad w_{m}^{* *}=\left(\frac{\beta_{m}^{2} \alpha_{m}^{2 n /(n+2)}}{2 \pi \mathcal{B}_{m}}\right)^{1 / 3} 2^{-\lambda_{m}}, \quad p_{m}^{*}=\beta_{m} \alpha_{m}^{\frac{n}{n+2}}, \\
& \beta_{m}=\left[\frac{2(2+n)^{2}}{n} \tan \left(\frac{\pi n}{2+n}\right)\right]^{1 /(2+n)}, \quad \bar{\delta}_{m}=\frac{2}{2+n}, \quad \alpha_{m}=\frac{2 n+2}{3 n+6}, \\
& \mathcal{B}_{m}=\mathcal{B}\left(\lambda_{m}, \bar{\delta}_{m}\right) \text { (equation (27)), } \quad \lambda_{m}=\lambda_{m}(n) \text { is shown in Figure 2(a). }
\end{aligned}
$$

\section{$\tilde{M}$-vertex}

\section{Dimensional form:}

$$
\begin{aligned}
& R_{\tilde{m}}(t)=0.4502 t^{1 / 4} \sqrt{\frac{Q_{0}}{C^{\prime}}}, \quad w_{\tilde{m}}(\rho, t)=w_{\tilde{m}}^{* *}\left(\frac{M^{\prime 4} Q_{0}^{2 n+4} t^{2-n}}{E^{\prime 4} C^{\prime 4-2 n}}\right)^{1 /(8+8 n)}(1+\rho)^{\lambda_{\tilde{m}}}(1-\rho)^{\bar{\delta}_{\tilde{m}}}, \\
& p_{\tilde{m}}(\rho, t)=p_{\tilde{m}}^{*}\left(\frac{M^{\prime 4} C^{\prime 6 n} E^{\prime 8 n+4}}{Q_{0}^{2 n} t^{3 n}}\right)^{1 /(8+8 n)} \mathcal{F}\left(\rho, \lambda_{\tilde{m}}, \bar{\delta}_{\tilde{m}}\right) .
\end{aligned}
$$

Normalised form:

$$
\begin{aligned}
& \gamma_{\tilde{m}}(\tau)=0.4502 \tau^{1 / 4} \phi^{-1 / 8}, \quad \Omega_{\tilde{m}}(\rho, \tau)=w_{\tilde{m}}^{* *}\left(\tau \phi^{-1 / 2}\right)^{(2-n) /(8+8 n)}(1+\rho)^{\lambda_{\tilde{m}}}(1-\rho)^{\bar{\delta}_{\tilde{m}}}, \\
& \Pi_{\tilde{m}}(\rho, \tau)=p_{\tilde{m}}^{*}\left(\phi^{1 / 2} / \tau\right)^{3 n /(8+8 n)} \mathcal{F}\left(\rho, \lambda_{\tilde{m}}, \bar{\delta}_{\tilde{m}}\right) .
\end{aligned}
$$

Parameters:

$$
\begin{aligned}
& w_{\tilde{m}}^{* *}=\beta_{\tilde{m}}\left(\frac{2}{\pi^{2}}\right)^{(n+2) /(4 n+4)} 2^{-\lambda_{\tilde{m}}}, \quad p_{\tilde{m}}^{*}=\beta_{\tilde{m}}\left(\frac{\pi^{2}}{2}\right)^{n /(4 n+4)}, \\
& \beta_{\tilde{m}}=\left[\frac{64(1+n)^{2}}{3 n(4+n)} \tan \left(\frac{3 \pi n}{4+4 n}\right)\right]^{1 /(2+2 n)}, \quad \bar{\delta}_{\tilde{m}}=\frac{4+n}{4+4 n}, \quad \lambda_{\tilde{m}}=\lambda_{\tilde{m}}(n) \text { is shown in Figure 4(a). }
\end{aligned}
$$

\section{$K$-vertex}

Dimensional form:

$$
R_{k}(t)=0.8546\left(\frac{E^{\prime} Q_{0} t}{K^{\prime}}\right)^{2 / 5}, \quad w_{k}(\rho, t)=0.6537\left(\frac{K^{\prime 4} Q_{0} t}{E^{\prime 4}}\right)^{1 / 5} \sqrt{1-\rho^{2}}, \quad p_{k}(t)=0.3004\left(\frac{K^{\prime 6}}{E^{\prime} Q_{0} t}\right)^{1 / 5} .
$$

Normalised form:

$$
\gamma_{k}(\tau)=0.8546 \tau^{2 / 5}, \quad \Omega_{k}(\rho, \tau)=0.6537 \tau^{1 / 5} \sqrt{1-\rho^{2}}, \quad \Pi_{k}(\tau)=0.3004 \tau^{1 / 5} .
$$

\section{$\tilde{K}$-vertex}

Dimensional form:

$$
R_{\tilde{k}}(t)=0.4502 t^{1 / 4} \sqrt{\frac{Q_{0}}{C^{\prime}}}, \quad w_{\tilde{k}}(\rho, t)=0.4744\left(\frac{K^{\prime 8} Q_{0}^{2} t}{C^{\prime 2} E^{\prime 8}}\right)^{1 / 8} \sqrt{1-\rho^{2}}, \quad p_{\tilde{k}}(t)=0.4139\left(\frac{C^{\prime 2} K^{\prime 8}}{Q_{0}^{2} t}\right)^{1 / 8} .
$$

Normalised form:

$$
\gamma_{\tilde{k}}(\tau)=0.4502 \tau^{1 / 4} \phi^{-1 / 8}, \quad \Omega_{\tilde{k}}(\rho, \tau)=0.4744 \tau^{1 / 8} \phi^{-1 / 16} \sqrt{1-\rho^{2}}, \quad \Pi_{\tilde{k}}(\tau)=0.4139 \tau^{-1 / 8} \phi^{1 / 16} .
$$




\section{$T$-vertex}

Dimensional form:

$$
\begin{aligned}
& R_{t}(t)=0.6349\left(\frac{t E^{\prime 1 / 2} Q_{0}}{\tau_{0}^{1 / 2}}\right)^{1 / 3}, \quad w_{t}(\rho, t)=1.5598\left(\frac{Q_{0} \tau_{0} t}{E^{\prime}}\right)^{1 / 3}(1+\rho)^{1.029}(1-\rho), \\
& p_{t}(\rho, t)=5.0133 \sqrt{\tau_{0} E^{\prime}} \mathcal{F}(\rho, 1.029,1) .
\end{aligned}
$$

Normalised form:

$$
\gamma_{t}(\tau)=0.6349 \tau^{1 / 3} \psi^{-1 / 6}, \quad \Omega_{t}(\rho, \tau)=1.5598 \tau^{1 / 3} \psi^{1 / 3}(1+\rho)^{1.029}(1-\rho), \quad \Pi_{t}(\rho, \tau)=5.0133 \sqrt{\psi} \mathcal{F}(\rho, 1.029,1) .
$$

\section{$\tilde{T}$-vertex}

\section{Dimensional form:}

$$
\begin{aligned}
& R_{\tilde{t}}(t)=0.4502 t^{1 / 4} \sqrt{\frac{Q_{0}}{C^{\prime}}}, \quad w_{\tilde{t}}(\rho, t)=1.0697\left(\frac{Q_{0} \sqrt{t} \tau_{0}}{C^{\prime} E^{\prime}}\right)^{1 / 2}(1+\rho)^{1.077}(1-\rho), \\
& p_{\tilde{t}}(\rho, t)=5.0128 \sqrt{E^{\prime} \tau_{0}} \mathcal{F}(\rho, 1.077,1) .
\end{aligned}
$$

Normalised form:

$$
\begin{aligned}
& \gamma_{\tilde{t}}(\tau)=0.4502 \tau^{1 / 4} \phi^{-1 / 8}, \quad \Omega_{\tilde{t}}(\rho, \tau)=1.0697 \tau^{1 / 4} \phi^{-1 / 8} \psi^{1 / 2}(1+\rho)^{1.077}(1-\rho), \\
& \Pi_{\tilde{t}}(\rho, \tau)=5.0128 \psi^{1 / 2} \mathcal{F}(\rho, 1.077,1) .
\end{aligned}
$$

\section{Validation of the approximate solution}

This section presents the validation procedure for the constructed approximate numerical solution (Section 3.2) with the help of the accurate approach (Section 3.1). We check its accuracy in terms of the radius, opening at the wellbore, pressure at the half-radius, and efficiency time histories carrying out simulations for different values of the governing parameters $\phi, \psi$ and the flow behaviour index $n$. In Section 4.1, we have already looked at the accuracy of the approximate method in the limiting propagation regimes, and here, the main focus is aimed at the transitions between them.

For the impermeable formation case, we analyse the set $n=\{1,0.75,0.3\}$ and for each value of the flow behaviour index, the computations are performed for $\psi=\left\{10^{-10}, 10^{-5}, 10^{0}, 10^{5}\right\}$. Figure 15 shows the crack properties related to $n=1$, and they are normalised by the storage-viscosity limiting solution. We estimate the relative differences between the fracture characteristics in the fully numerical and approximate solutions according to the relation: $\delta_{A}=$ $\left|A_{\text {numer }}-A_{\text {appr }}\right| /\left|A_{\text {numer }}\right|, A=\{\gamma, \Omega(0), \Pi(0.5)\}$, find the maximum and average quantities of $\delta_{A}$ across the time domain $\left[\tau_{\min }, \tau_{\max }\right]$. We summarise the calculations in Table 3 together with $\tau_{\min }$ and $\tau_{\max }$ values. Using the metrics from Table 3 in combination with Figure 15, we conclude that the approximate methodology provides reasonably accurate predictions of the radius evolution. The average error in the wellbore opening calculations is relatively small (the peak value among the considered cases is $2 \%$ ); however, the maximum difference can reach $16 \%$ in the transition zone between $M$ and $T$ vertex solutions. The pressure profiles are computed with the average error lying within the interval $(2,11) \%$, and there is a tendency of the difference growth with an increase of the yield stress number. Similarly to the wellbore opening, the maximum error for $\Pi(0.5)$ is reached between the viscosity and yield-stress regimes, and it is up to $16 \%$. Note that $\tau_{\max }$ is not constant for all these cases, and it is linked with the ability of the fully numerical solution to perform calculations inside the yield-stress dominated regime where almost the whole fracture is occupied by the plug zone, and the fluid flow velocity approaches zero.

Let us move on to the validation of the approximate solution for crack propagation in a permeable rock. Both power-law $(\psi=0)$ and Herschel-Bulkley $(\psi=1)$ rheologies are analysed for $n=\{1,0.75,0.3\}$, and we apply the following set of the leak-off number values: $\phi=\left\{10^{-20}, 10^{-10}, 10^{-5}, 10^{0}, 10^{5}, 10^{10}\right\}$. Figure 16 shows the comparison for $n=1, \psi=1$ (the radius, opening, and pressure profiles are normalised by the $M$ vertex solution), while the relative 

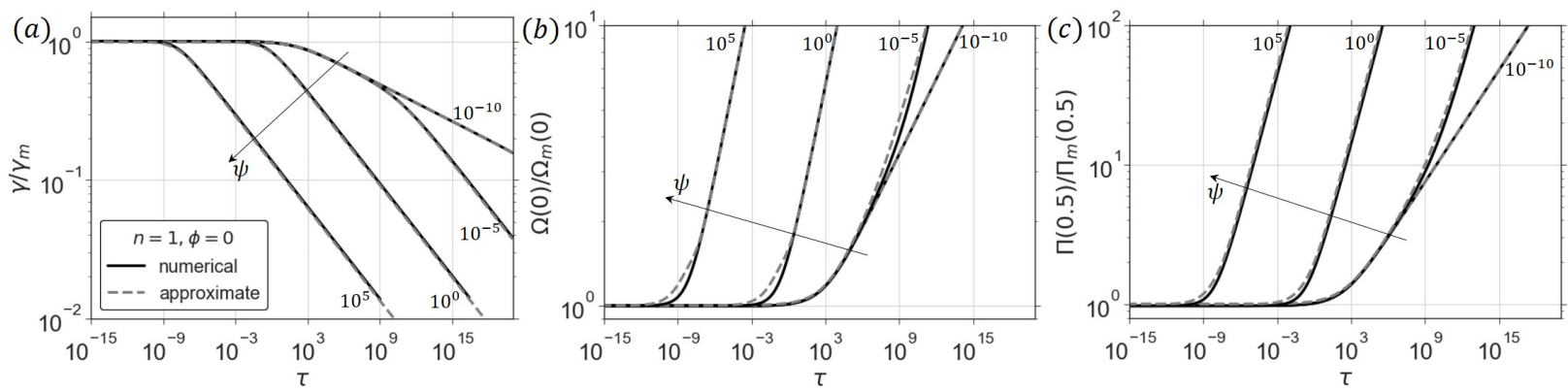

Figure 15: The comparison between the fully numerical (the solid black lines) and approximate (the dashed grey lines) numerical solutions corresponding to $n=1$ flow behaviour index, an impermeable rock $(\phi=0)$, and the set of the yield stress number values: $\psi=\left\{10^{-10}, 10^{-5}, 10^{0}, 10^{5}\right\}$. Three time-dependent crack parameters are considered: radius (a), opening at the wellbore (b), and pressure at the half-radius (c), and all properties are normalised by the storage-viscosity limiting solution.

Table 3

Table contains the values (in \%) of the relative differences $\delta_{A}$ between the fully numerical and approximate numerical solutions in terms of radius $\gamma$, aperture at the wellbore $\Omega(0)$, and pressure at the half-radius $\Pi(0.5)$. Two values of $\delta_{A}$ are written in each cell corresponding to the aforementioned parameters: maximum and average across the considered time span $\tau \in\left[\tau_{\min }, \tau_{\max }\right]$. We analyse solutions for $n=\{0.3,0.75,1\}$ and the set of the yield stress number values $\psi=\left\{10^{-10}, 10^{-5}, 10^{0}, 10^{5}\right\}$.

\begin{tabular}{|c|c|c|c|c|c|c|c|}
\hline \multirow{2}{*}{ Average } & \multicolumn{7}{|c|}{${ }_{A}=\left|A_{\text {numer }}-A_{\text {appr }}\right| /\left|A_{\text {numer }}\right|($ in $\%)$ across time interval $\left[\tau_{\min }, \tau_{\max }\right]$} \\
\hline & \multicolumn{7}{|c|}{$\phi=0$} \\
\hline \multirow[t]{2}{*}{ Parameter $(\mathrm{A}) \backslash \psi$} & \multicolumn{2}{|c|}{$10^{-10}$} & \multicolumn{2}{|c|}{$10^{-5}$} & $10^{0}$ & \multicolumn{2}{|c|}{$10^{5}$} \\
\hline & \multicolumn{7}{|c|}{$n=1, \tau_{\min }=10^{-15}$} \\
\hline$\tau_{\max }$ & \multicolumn{2}{|c|}{$10^{20}$} & \multicolumn{2}{|c|}{$10^{20}$} & $2.5 \cdot 10^{16}$ & \multicolumn{2}{|c|}{$10^{9}$} \\
\hline$\gamma$ & 0.3 & 0.7 & 1.0 & 2.8 & \begin{tabular}{l|l|l}
1.7 & 2.8
\end{tabular} & 2.2 & \\
\hline$\Omega(0)$ & 0.6 & 9.3 & 2.1 & 15.8 & \begin{tabular}{l|l}
1.1 & 13.0
\end{tabular} & 1.3 & 12.7 \\
\hline \multirow[t]{2}{*}{$\Pi(0.5)$} & 1.7 & 7.0 & 6.3 & 15.5 & \begin{tabular}{l|l}
8.1 & 12.3 \\
\end{tabular} & 9.8 & 12.3 \\
\hline & \multicolumn{7}{|c|}{$n=0.75, \tau_{\min }=10^{-15}$} \\
\hline$\tau_{\max }$ & \multicolumn{2}{|c|}{$10^{20}$} & \multicolumn{2}{|c|}{$10^{20}$} & $2.4 \cdot 10^{14}$ & \multicolumn{2}{|c|}{$1.8 \cdot 10^{5}$} \\
\hline$\gamma$ & 0.4 & 0.7 & 1.0 & 2.8 & \begin{tabular}{l|l}
1.6 & 2.8
\end{tabular} & 2.2 & 2.8 \\
\hline$\Omega(0)$ & 0.9 & 7.9 & 1.8 & 12.4 & \begin{tabular}{l|l}
1.0 & 9.7
\end{tabular} & 1.3 & 9.5 \\
\hline \multirow[t]{2}{*}{$\Pi(0.5)$} & 2.4 & 5.4 & 6.3 & 12.7 & \begin{tabular}{l|l}
8.0 & 12.2
\end{tabular} & 10.2 & 12.1 \\
\hline & \multicolumn{7}{|c|}{$n=0.3, \tau_{\min }=10^{-20}$} \\
\hline$\tau_{\max }$ & \multicolumn{2}{|c|}{$10^{20}$} & \multicolumn{2}{|c|}{$10^{20}$} & $10^{20}$ & \multicolumn{2}{|c|}{$9.5 \cdot 10^{7}$} \\
\hline$\gamma$ & 0.8 & 1.5 & 0.9 & 2.3 & \begin{tabular}{l|l|l}
1.5 & 2.9 \\
\end{tabular} & 2.2 & 2.9 \\
\hline$\Omega(0)$ & 1.8 & 6.2 & 2.2 & 6.2 & 2.1 & 1.8 & 12.5 \\
\hline$\Pi(0.5)$ & 5.5 & 7.8 & 6.1 & 10.1 & 12.1 & 10.7 & 12.1 \\
\hline
\end{tabular}

differences $\delta_{A}, A=\{\gamma, \Omega(0), \Pi(0.5), \eta\}$ (average and maximum values over the time span $\left[\tau_{\min }, \tau_{\max }\right]$ ) are summarised in Table 4. We also note that the upper boundary of the time interval is limited now by the ability of the fully numerical approach to handle the crack propagation with almost zero efficiency (apart from the obstacle connected with the plug zone mentioned before for $\phi=0$ investigations).

By looking at the results shown in Table 4(a) for the power-law fluid, we find out that the approximate approach estimates the radius and efficiency evolution accurately with the average and maximum errors no more than $1 \%$ and $3 \%$, respectively. Approximately the same scores are noticed for $\Omega(0)$ when $n=1$ and 0.75 . In contrast, the quality of estimations degrades for $n=0.3$ where the average and maximum differences are approximately $3 \%$ and $6 \%$. The approximate methodology provides values of the pressure at the half-radius with the average relative error less than $5 \%$.

Further, we turn to the case $\psi=1$ (see metrics in Table 4 and Figure 16 for $n=1$ ), and here, the main trends 

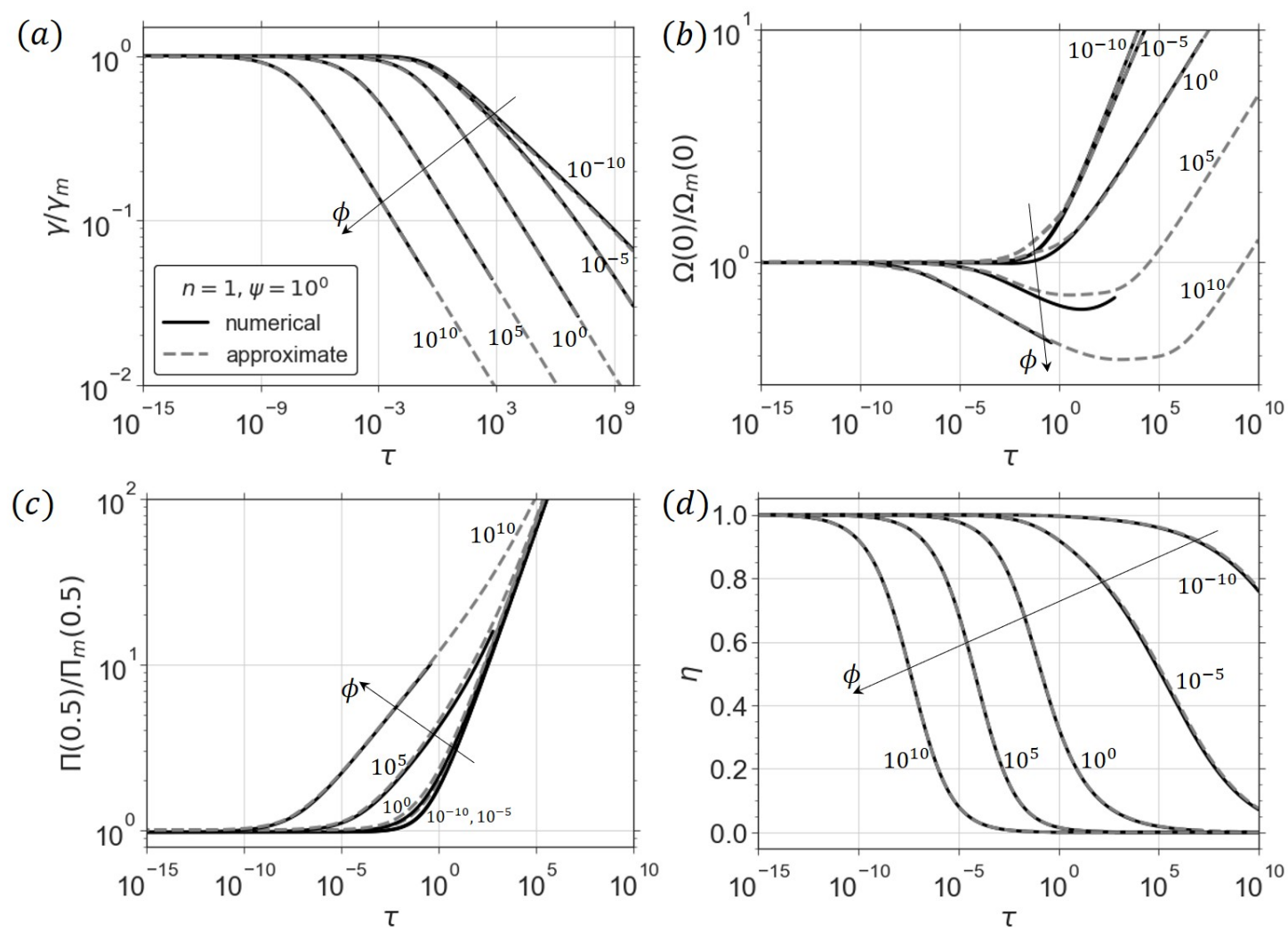

Figure 16: The comparison between the fully numerical (the solid black lines) and approximate (the dashed grey lines) numerical solutions corresponding to $n=1$ flow behaviour index, the set of the leak-off number values $\phi=$ $\left\{10^{-10}, 10^{-5}, 10^{0}, 10^{5}, 10^{10}\right\}$, and $\psi=1$ yield stress number. Four time-dependent crack parameters are considered: radius (a), opening at the wellbore (b), pressure at the half-radius (c), and efficiency (d). The solution profiles in panels (a)-(c) are normalised by the storage-viscosity limiting solution.

are similar to the analysis for the power-law fluid. The average errors of the radius and efficiency computations are smaller than $1.5 \%$, and the maximum differences reach $3 \%$ and $6 \%$, correspondingly. The approximate approach gives the wellbore opening with the average accuracy less than $4 \%$, while the peak differences are noticed between $\tilde{M}$ and $\tilde{T}$ regimes and comprise values up to $16 \%$. The average precision of the pressure calculations is approximately $7 \%$, and the difference between the fully numerical and approximate solutions for this parameter can achieve $13 \%$.

\section{References}

Abe, H., Keer, L., Mura, T., 1976. Growth rate of a penny-shaped crack in hydraulic fracturing of rocks, 2. Journal of Geophysical Research 81, 6292-6298.

Adachi, J., Detournay, E., 2002. Self-similar solution of a plane-strain fracture driven by a power-law fluid. International Journal for Numerical and Analytical Methods in Geomechanics 26, 579-604.

Adachi, J., Siebrits, E., Peirce, A., Desroches, J., 2007. Computer simulation of hydraulic fractures. International Journal of Rock Mechanics and Mining Sciences 44, 739-757.

Adachi, J.I., Detournay, E., Peirce, A.P., 2010. Analysis of the classical pseudo-3d model for hydraulic fracture with equilibrium height growth across stress barriers. International Journal of Rock Mechanics and Mining Sciences 47, 625-639.

Arin, K., Erdogan, F., 1971. Penny-shaped crack in an elastic layer bonded to dissimilar half spaces. International Journal of Engineering Science 9, 213-232.

Barbati, A.C., Desroches, J., Robisson, A., McKinley, G.H., 2016. Complex fluids and hydraulic fracturing. Annual review of chemical and biomolecular engineering 7, 415-453.

Batchelor, G.K., 1967. An Introduction to Fluid Dynamics. Cambridge University Press, Cambridge UK.

Bessmertnykh, A., Dontsov, E., Ballarini, R., 2020. The effects of proppant on the near-front behavior of a hydraulic fracture. Engineering Fracture Mechanics, 107110. 
Bessmertnykh, A.O., Dontsov, E.V., 2019. A semi-infinite hydraulic fracture driven by a herschel-bulkley fluid. Journal of Applied Mechanics 86. Bingham, E.C., 1922. Fluidity and plasticity. volume 2. McGraw-Hill.

Bird, R.B., Armstrong, R.C., Hassager, O., 1987. Dynamics of polymeric liquids. vol. 1: Fluid mechanics .

Brodkey, R.S., Brodkey, 1967. The phenomena of fluid motions .

Bunger, A.P., Detournay, E., 2007. Early-time solution for a radial hydraulic fracture. Journal of engineering mechanics 133, 534-540.

Bunger, A.P., Detournay, E., 2008. Experimental validation of the tip asymptotics for a fluid-driven crack. Journal of the Mechanics and Physics of Solids 56, 3101-3115.

Bunger, A.P., Detournay, E., Garagash, D.I., 2005. Toughness-dominated hydraulic fracture with leak-off. International journal of fracture 134, $175-190$.

Carreau, P.J., 1972. Rheological equations from molecular network theories. Transactions of the Society of Rheology 16, 99-127.

Carter, E., 1957. Optimum fluid characteristics for fracture extension, in: Howard, G.C., Fast, C.R. (Eds.), Drilling and Production Practices, pp. 261-270.

Cleary, M., Wong, S., 1985. Numerical simulation of unsteady fluid flow and propagation of a circular hydraulic fracture. International Journal for Numerical and Analytical Methods in Geomechanics 9, 1-14.

Cross, M.M., 1965. Rheology of non-newtonian fluids: a new flow equation for pseudoplastic systems. Journal of colloid science $20,417-437$.

Desroches, J., Detournay, E., Lenoach, B., Papanastasiou, P., Pearson, J.R.A., Thiercelin, M., Cheng, A., 1994. The crack tip region in hydraulic fracturing. Proceedings of the Royal Society of London. Series A: Mathematical and Physical Sciences 447, $39-48$.

Detournay, E., 2016. Mechanics of hydraulic fractures. Annual Review of Fluid Mechanics 48, 311-339.

Detournay, E., Garagash, D., 2003. The near-tip region of a fluid-driven fracture propagating in a permeable elastic solid. Journal of Fluid Mechanics 494, 1-32.

Dontsov, E., 2016a. An approximate solution for a penny-shaped hydraulic fracture that accounts for fracture toughness, fluid viscosity and leak-off. Royal Society open science 3, 160737.

Dontsov, E., 2016b. Propagation regimes of buoyancy-driven hydraulic fractures with solidification. Journal of Fluid Mechanics 797, 1-28.

Dontsov, E., 2016c. Tip region of a hydraulic fracture driven by a laminar-to-turbulent fluid flow. Journal of Fluid Mechanics 797.

Dontsov, E., 2017. An approximate solution for a plane strain hydraulic fracture that accounts for fracture toughness, fluid viscosity, and leak-off. International Journal of Fracture 205, 221-237.

Dontsov, E., Bunger, A., Abell, B., Suarez-Rivera, R., 2019a. Ultrafast hydraulic fracturing model for optimizing cube development, in: Proceedings of the Unconventional Resources Technology Conference, doi:10.15530/urtec-2019-884.

Dontsov, E., Bunger, A., Abell, B., Suarez-Rivera, R., 2019b. Ultrafast hydraulic fracturing model for optimizing cube development, in: Proceedings of the Unconventional Resources Technology Conference, doi:10.15530/urtec-2019-884.

Dontsov, E., Kresse, O., 2018. A semi-infinite hydraulic fracture with leak-off driven by a power-law fluid. Journal of Fluid Mechanics 837, 210-229.

Dontsov, E., Peirce, A., 2015a. An enhanced pseudo-3d model for hydraulic fracturing accounting for viscous height growth, non-local elasticity, and lateral toughness. Engineering Fracture Mechanics 142, 116-139.

Dontsov, E., Peirce, A., 2015b. A non-singular integral equation formulation to analyse multiscale behaviour in semi-infinite hydraulic fractures. Journal of Fluid Mechanics 781.

Dontsov, E., Peirce, A., 2017a. Modeling planar hydraulic fractures driven by laminar-to-turbulent fluid flow. International Journal of Solids and Structures 128, $73-84$.

Dontsov, E., Peirce, A., 2017b. A multiscale implicit level set algorithm (ilsa) to model hydraulic fracture propagation incorporating combined viscous, toughness, and leak-off asymptotics. Computer Methods in Applied Mechanics and Engineering 313, 53-84.

Dontsov, E.V., 2019. Scaling laws for hydraulic fractures driven by a power-law fluid in homogeneous anisotropic rocks. International Journal for Numerical and Analytical Methods in Geomechanics 43, 519-529.

Economides, M., Oligney, R., Valkó, P., 2002. Unified fracture design: bridging the gap between theory and practice. Orsa Press.

Economides, M.J., Nolte, K.G., et al., 1989. Reservoir stimulation. volume 2. Prentice Hall Englewood Cliffs, NJ.

Faroughi, S.A., Pruvot, A.J.C.J., McAndrew, J., 2018. The rheological behavior of energized fluids and foams with application to hydraulic fracturing. Journal of Petroleum Science and Engineering 163, 243-263.

Fu, C., Liu, N., 2019. Waterless fluids in hydraulic fracturing-a review. Journal of Natural Gas Science and Engineering 67, 214-224.

Garagash, D., 2006a. Transient solution for a plane-strain fracture driven by a shear-thinning, power-law fluid. International journal for numerical and analytical methods in geomechanics 30, 1439-1475.

Garagash, D., Detournay, E., 2000. The tip region of a fluid-driven fracture in an elastic medium. J. Appl. Mech. 67, $183-192$.

Garagash, D.I., 2000. Hydraulic fracture propagation in elastic rock with large toughness, in: Girard, J., Liebman, M., Breeds, C., Doe, T. (Eds.), Pacific Rocks 2000 - Proc. 4th North American Rock Mechanics Symp., Balkema, Rotterdam. pp. 221-228.

Garagash, D.I., 2006b. Plane-strain propagation of a fluid-driven fracture during injection and shut-in: Asymptotics of large toughness. Engineering fracture mechanics $73,456-481$.

Garagash, D.I., 2006c. Propagation of a plane-strain hydraulic fracture with a fluid lag: Early-time solution. International journal of solids and structures 43, 5811-5835.

Garagash, D.I., 2019. Cohesive-zone effects in hydraulic fracture propagation. Journal of the Mechanics and Physics of Solids $133,103727$.

Garagash, D.I., Detournay, E., Adachi, J.I., 2011. Multiscale tip asymptotics in hydraulic fracture with leak-off. J. Fluid Mech. 669, $260-297$. doi:10.1017/S002211201000501X.

Garagash, D.I., Sarvaramini, E., 2012. Equilibrium of a pressurized plastic fluid in a wellbore crack. International journal of solids and structures 49, 197-212.

Garagash, I.A., Osiptsov, A.A., Boronin, S.A., 2019. Dynamic bridging of proppant particles in a hydraulic fracture. International Journal of Engineering Science 135, 86-101. 
Geertsma, J., De Klerk, F., et al., 1969. A rapid method of predicting width and extent of hydraulically induced fractures. Journal of Petroleum Technology 21, 1-571.

Gu, M., Mohanty, K., 2015. Rheology of polymer-free foam fracturing fluids. Journal of Petroleum Science and Engineering 134, 87-96.

Herschel, W., Bulkley, R., 1926. Measurement of consistency as applied to rubber-benzene solutions, in: Am. Soc. Test Proc, pp. 621-633.

Irvin, G.R., 1957. Analysis of stresses and strains near the end of a crack traversing a plate. ASME J. Appl. Mech. 24, 361-364.

Kanin, E., Garagash, D., Osiptsov, A., 2020a. Turbulent flow effects in a slickwater fracture propagation in permeable rock, in: ECMOR XVII, European Association of Geoscientists \& Engineers. pp. 1-18.

Kanin, E.A., Dontsov, E.V., Garagash, D.I., Osiptsov, A.A., 2020b. A radial hydraulic fracture with pressure-dependent leak-off. Journal of the Mechanics and Physics of Solids, 104062.

Kanin, E.A., Garagash, D., Osiptsov, A., 2020c. Radial fracture in permeable rock driven by turbulent-laminar flow, in: AGU Fall Meeting 2020, AGU.

Kanin, E.A., Garagash, D.I., Osiptsov, A.A., 2020d. The near-tip region of a hydraulic fracture with pressure-dependent leak-off and leak-in. Journal of Fluid Mechanics 892, A31. doi:10.1017/jfm.2020.193.

Khristianovic, S., Zheltov, Y., 1955. Formation of vertical fractures by means of highly viscous fluids, in: Proc. 4th world petroleum congress, Rome, pp. 579-586.

Kovalyshen, Y., 2010. Fluid-Driven Fracture in Poroelastic Medium. Ph.D. thesis. University of Minnesota.

Kovalyshen, Y., Detournay, E., et al., 2013. Fluid-driven fracture in a poroelastic rock, in: ISRM International Conference for Effective and Sustainable Hydraulic Fracturing, International Society for Rock Mechanics and Rock Engineering.

Lavrov, A., 2015. Flow of truncated power-law fluid between parallel walls for hydraulic fracturing applications. Journal of Non-Newtonian Fluid Mechanics 223, 141-146.

Lecampion, B., Bunger, A., Zhang, X., 2018. Numerical methods for hydraulic fracture propagation: a review of recent trends. Journal of natural gas science and engineering 49, 66-83.

Lecampion, B., Desroches, J., 2015. Simultaneous initiation and growth of multiple radial hydraulic fractures from a horizontal wellbore. Journal of the Mechanics and Physics of Solids 82, 235-258.

Lecampion, B., Zia, H., 2019. Slickwater hydraulic fracture propagation: near-tip and radial geometry solutions. Journal of Fluid Mechanics 880 , $514-550$.

Lee, S.H.A.T., Lee, J., 1990. Three-dimensional modeling of hydraulic fractures in layered media: Part I-finite element formulations. J. Energy Resour. Technol. 112, 1-9.

Lenoach, B., 1995. The crack tip solution for hydraulic fracturing in a permeable solid. Journal of the Mechanics and Physics of Solids 43 , $1025-1043$.

Lister, J.R., 1990. Buoyancy-driven fluid fracture: the effects of material toughness and of low-viscosity precursors. Journal of Fluid Mechanics 210, 263-280.

Liu, D., Lecampion, B., 2021. Propagation of a plane-strain hydraulic fracture accounting for a rough cohesive zone. Journal of the Mechanics and Physics of Solids 149, 104322.

Liu, D., Lecampion, B., Garagash, D.I., 2019. Propagation of a fluid-driven fracture with fracture length dependent apparent toughness. Engineering Fracture Mechanics 220, 106616.

Madyarova, M.V., 2003. Fluid-driven penny-shaped fracture in elastic medium. Master's thesis. University of Minnesota.

Molchanov, V., Aleshina, A., Shibaev, A., et al., 2021. Insights into rheology of guar-based fracturing fluids from high-precision measurements: does it have a real yield stress? Journal of Petroleum Science and Engineering TBD, (under consideration).

Morris, J.F., 2020a. Shear thickening of concentrated suspensions: Recent developments and relation to other phenomena. Annual Review of Fluid Mechanics 52, 121-144.

Morris, J.F., 2020b. Toward a fluid mechanics of suspensions. Physical Review Fluids 5, 110519.

Moukhtari, F.E., Lecampion, B., 2018. A semi-infinite hydraulic fracture driven by a shear-thinning fluid. Journal of Fluid Mechanics 838, 573-605.

Nguyen, H.T., Lee, J.H., Elraies, K.A., 2020. A review of pkn-type modeling of hydraulic fractures. Journal of Petroleum Science and Engineering , 107607.

Nordgren, R., et al., 1972. Propagation of a vertical hydraulic fracture. Society of Petroleum Engineers Journal 12, 306-314.

Osiptsov, A., Garagash, I., Boronin, S., Tolmacheva, K., Lezhnev, K., Paderin, G., 2019. Impact of flowback dynamics on fracture conductivity. Journal of Petroleum Science and Engineering, 106822.

Osiptsov, A.A., 2017. Fluid mechanics of hydraulic fracturing: a review. Journal of petroleum science and engineering 156, 513-535.

Peck, D., Wrobel, M., Perkowska, M., Mishuris, G., 2018a. Fluid velocity based simulation of hydraulic fracture: a penny shaped model-part i: the numerical algorithm. Meccanica 53, 3615-3635.

Peck, D., Wrobel, M., Perkowska, M., Mishuris, G., 2018b. Fluid velocity based simulation of hydraulic fracture-a penny shaped model. part ii: new, accurate semi-analytical benchmarks for an impermeable solid. Meccanica 53, 3637-3650.

Peirce, A., 2015. Modeling multi-scale processes in hydraulic fracture propagation using the implicit level set algorithm. Computer Methods in Applied Mechanics and Engineering 283, 881-908.

Peirce, A., Detournay, E., 2008. An implicit level set method for modeling hydraulically driven fractures. Computer Methods in Applied Mechanics and Engineering 197, 2858-2885.

Perkins, T., Kern, L., et al., 1961. Widths of hydraulic fractures. Journal of Petroleum Technology 13, 937-949.

Perkowska, M., Wrobel, M., Mishuris, G., 2016. Universal hydrofracturing algorithm for shear-thinning fluids: particle velocity based simulation. Computers and Geotechnics 71, 310-337.

Rivalta, E., Taisne, B., Bunger, A., Katz, R., 2015. A review of mechanical models of dike propagation: Schools of thought, results and future directions. Tectonophysics $638,1-42$.

Rubin, A.M., 1993. Tensile fracture of rock at high confining pressure: implications for dike propagation. Journal of Geophysical Research: Solid 
Earth 98, 15919-15935.

Savitski, A., Detournay, E., 2002. Propagation of a penny-shaped fluid-driven fracture in an impermeable rock: asymptotic solutions. International journal of solids and structures 39, 6311-6337.

Settari, A., Cleary, M.P., et al., 1986. Development and testing of a pseudo-three-dimensional model of hydraulic fracture geometry. SPE Production Engineering 1, 449-466.

Spence, D., Turcotte, D., 1985. Magma-driven propagation of cracks. Journal of Geophysical Research: Solid Earth 90, 575-580.

Stickel, J.J., Powell, R.L., 2005. Fluid mechanics and rheology of dense suspensions. Annu. Rev. Fluid Mech. 37, $129-149$.

Suarez-Rivera, R., Dontsov, E., Abell, B., 2019. Quantifying the induced stresses during stacked, multi-stage, multi- well completions, to define stimulation sequencing and optimize pad productivity, in: Proceedings of the Unconventional Resources Technology Conference, doi:10.15530/urtec2019-892.

Tsai, V.C., Rice, J.R., 2010. A model for turbulent hydraulic fracture and application to crack propagation at glacier beds. Journal of Geophysical Research: Earth Surface 115.

Vandamme, L., Curran, J., 1989. A three-dimensional hydraulic fracturing simulator. Int. J. Numer. Meth. Eng. 28, 909-927.

van der Veen, C.J., 2007. Fracture propagation as means of rapidly transferring surface meltwater to the base of glaciers. Geophysical Research Letters 34.

Viesca, R.C., Garagash, D.I., 2018. Numerical methods for coupled fracture problems. Journal of the Mechanics and Physics of Solids 113, 13-34. Virtanen, P., Gommers, R., Oliphant, T.E., Haberland, M., Reddy, T., Cournapeau, D., Burovski, E., Peterson, P., Weckesser, W., Bright, J., et al., 2020. Scipy 1.0: fundamental algorithms for scientific computing in python. Nature methods 17, 261-272.

Vishkai, M., Gates, I., 2019. On multistage hydraulic fracturing in tight gas reservoirs: Montney formation, alberta, canada. Journal of Petroleum Science and Engineering 174, 1127-1141.

Wrobel, M., 2020. On the application of simplified rheological models of fluid in the hydraulic fracture problems. International Journal of Engineering Science 150, 103275.

Wrobel, M., Mishuris, G., 2015. Hydraulic fracture revisited: Particle velocity based simulation. International Journal of Engineering Science 94, 23-58.

Wrobel, M., Mishuris, G., Papanastasiou, P., 2020. On the influence of fluid rheology on hydraulic fracture. International Journal of Engineering Science $158,103426$.

Zia, H., Lecampion, B., 2020. Pyfrac: A planar 3d hydraulic fracture simulator. Computer Physics Communications , 107368.

Zolfaghari, N., Bunger, A., 2019. Numerical model for a penny-shaped hydraulic fracture driven by laminar/turbulent fluid in an impermeable rock. International Journal of Solids and Structures 158, 128-140. 


\section{Table 4}

Tables contain the values (in \%) of the relative differences $\delta_{A}$ between the fully numerical and approximate solutions in terms of radius $\gamma$, aperture at the wellbore $\Omega(0)$, pressure at the half-radius $\Pi(0.5)$, and efficiency $\eta$. Two values of $\delta_{A}$ are written in each cell corresponding to the aforementioned parameters: maximum and average across the considered time span $\tau \in\left[\tau_{\min }, \tau_{\max }\right]$. We analyse solutions for $n=\{0.3,0.75,1\}$, and for each value of the flow behaviour index, both power-law $\left(\psi=0\right.$, table (a)) and Herschel-Bulkley $\left(\psi=10^{\circ}\right.$, table (b)) rheologies are examined. In turn, the set of the leak-off number values $\phi=\left\{10^{-20}, 10^{-10}, 10^{-5}, 10^{0}, 10^{5}, 10^{10}\right\}$ is applied for calculations for each $n$ and $\psi$.

(a) $\psi=0$

\begin{tabular}{|c|c|c|c|c|c|c|c|c|c|c|c|c|}
\hline \multicolumn{13}{|c|}{ Average $\mid$ Maximum $\delta_{A}=\left|A_{\text {numer }}-A_{\text {appr }}\right| /\left|A_{\text {numer }}\right|$ (in \%) across time interval $\left[\tau_{\min }, \tau_{\max }\right]$} \\
\hline \multirow{2}{*}{ Parameter $(\mathrm{A}) \backslash \phi$} & \multicolumn{2}{|c|}{$10^{-20}$} & \multicolumn{2}{|c|}{$10^{-10}$} & \multicolumn{2}{|c|}{$10^{-5}$} & \multicolumn{2}{|c|}{$10^{0}$} & \multicolumn{2}{|c|}{$10^{5}$} & \multicolumn{2}{|c|}{$10^{10}$} \\
\hline & \multicolumn{12}{|c|}{$n=1, \tau_{\min }=10^{-15}$} \\
\hline$\tau_{\max }$ & \multicolumn{2}{|c|}{$10^{10}$} & \multicolumn{2}{|c|}{$10^{10}$} & \multicolumn{2}{|c|}{$9.5 \cdot 10^{8}$} & \multicolumn{2}{|c|}{$1.1 \cdot 10^{6}$} & \multicolumn{2}{|c|}{$1.1 \cdot 10^{3}$} & \multicolumn{2}{|c|}{$9.1 \cdot 10^{-1}$} \\
\hline$\gamma$ & 0.4 & 0.7 & 0.5 & 0.7 & 0.5 & 0.7 & 0.6 & 0.8 & 0.6 & 0.8 & 0.5 & 0.8 \\
\hline$\Omega(0)$ & 0.3 & 0.8 & 0.3 & 0.7 & 0.4 & 1.2 & 0.4 & 1.6 & 0.4 & 1.5 & 0.4 & 1.6 \\
\hline$\Pi(0.5)$ & 2.0 & 3.1 & 2.0 & 3.1 & 2.2 & 2.8 & 2.4 & 2.8 & 1.9 & 2.4 & 1.6 & 2.4 \\
\hline$\eta$ & 0.0 & 0.0 & 0.2 & 1.2 & 0.3 & 1.4 & 0.2 & 1.0 & 0.2 & 0.6 & 0.2 & 0.6 \\
\hline & \multicolumn{12}{|c|}{$n=0.75, \tau_{\min }=10^{-15}$} \\
\hline$\tau_{\max }$ & \multicolumn{2}{|c|}{$10^{10}$} & \multicolumn{2}{|c|}{$10^{10}$} & \multicolumn{2}{|c|}{$10^{10}$} & \multicolumn{2}{|c|}{$2.2 \cdot 10^{6}$} & \multicolumn{2}{|c|}{$8.1 \cdot 10^{2}$} & \multicolumn{2}{|c|}{$1.9 \cdot 10^{-1}$} \\
\hline$\gamma$ & 0.5 & 0.7 & 0.5 & 0.7 & 0.5 & 0.7 & 0.6 & 0.7 & & 0.8 & 0.5 & 0.7 \\
\hline$\Omega(0)$ & 0.7 & 1.5 & 0.6 & 1.6 & 0.6 & 2.3 & 0.6 & 2.1 & 0.5 & 1.7 & 0.5 & 1.6 \\
\hline$\Pi(0.5)$ & 3.0 & 3.8 & 3.0 & 3.8 & 3.1 & 3.9 & 2.9 & 3.4 & 2.4 & 2.9 & 1.9 & 2.8 \\
\hline$\eta$ & 0.0 & 0.0 & 0.3 & 1.7 & 0.6 & 2.0 & 0.3 & 1.6 & 0.1 & 0.4 & 0.1 & 0.4 \\
\hline & \multicolumn{12}{|c|}{$n=0.3, \tau_{\min }=10^{-20}$} \\
\hline$\tau_{\max }$ & \multicolumn{2}{|c|}{$10^{10}$} & \multicolumn{2}{|c|}{$10^{10}$} & \multicolumn{2}{|c|}{$10^{10}$} & 6.2 & $10^{6}$ & \multicolumn{2}{|c|}{$1.2 \cdot 10^{2}$} & \multicolumn{2}{|c|}{$4.5 \cdot 10^{-3}$} \\
\hline$\gamma$ & 0.6 & 1.4 & 0.5 & 0.9 & 0.3 & 0.9 & 0.3 & 0.9 & 0.2 & 0.9 & 0.1 & 0.8 \\
\hline$\Omega(0)$ & 2.4 & 6.2 & 2.5 & 6.2 & 2.6 & 6.2 & 3.0 & 6.2 & 3.4 & 5.6 & 3.0 & 4.6 \\
\hline$\Pi(0.5)$ & 5.3 & 7.8 & 5.1 & 7.8 & 4.9 & 7.7 & 4.8 & 7.8 & 4.4 & 7.2 & 3.4 & 6.4 \\
\hline$\eta$ & 0.0 & 0.0 & 0.2 & 2.4 & 0.5 & 3.0 & 0.5 & 2.5 & 0.5 & 1.8 & 0.8 & 1.8 \\
\hline
\end{tabular}

(b) $\psi=10^{0}$

\begin{tabular}{|c|c|c|c|c|c|c|c|}
\hline Average $\mid$ Maximum $\delta_{A}=\left|A_{\text {numer }}-A_{\text {appr }}\right| /\left|A_{\text {numer }}\right|$ (in \%) across time interval $\left[\tau_{\min }, \tau_{\max }\right]$ \\
\hline Parameter $(\mathrm{A}) \backslash \phi$ & $10^{-20}$ & $10^{-10}$ & \multicolumn{1}{c|}{$10^{-5}$} & \multicolumn{1}{c|}{$10^{0}$} & \multicolumn{2}{c|}{$10^{5}$} & $10^{10}$ \\
\hline & \multicolumn{7}{c|}{$n=1, \tau_{\min }=10^{-15}$} \\
\hline$\tau_{\max }$ & $10^{10}$ & $10^{10}$ & $10^{10}$ & $1.5 \cdot 10^{7}$ & $6.1 \cdot 10^{2}$ & $4 \cdot 10^{-1}$ \\
\hline$\gamma$ & $1.5 \mid 2.8$ & $1.4 \mid 2.7$ & $1.1 \mid 2.1$ & $0.7 \mid 1.1$ & $0.6 \mid 0.9$ & $0.5 \mid 0.8$ \\
\hline$\Omega(0)$ & $1.3 \mid 13.0$ & $1.4 \mid 12.9$ & $1.5 \mid 12.7$ & $1.4 \mid 8.5$ & $3.4 \mid 15.7$ & $0.5 \mid 2.0$ \\
\hline$\Pi(0.5)$ & $7.1 \mid 12.3$ & $7.1 \mid 12.3$ & $6.9 \mid 12.2$ & $5.9 \mid 11.1$ & $4.2 \mid 12.8$ & $1.8 \mid 2.4$ \\
\hline$\eta$ & $0.0 \mid 0.0$ & $0.1 \mid 1.1$ & $1.0 \mid 5.3$ & $1.2 \mid 6.2$ & $1.0 \mid 4.5$ & $0.3 \mid 1.1$ \\
\hline \multicolumn{7}{|c|}{$n=0.75, \tau_{\min }=10^{-15}$} \\
\hline$\tau_{\max }$ & $10^{10}$ & $10^{10}$ & $10^{10}$ & $2.4 \cdot 10^{8}$ & $4 \cdot 10^{2}$ & $10^{-1}$ \\
\hline$\gamma$ & $1.4 \mid 2.8$ & $1.4 \mid 2.7$ & $1.0 \mid 2.0$ & $0.6 \mid 0.9$ & $0.6 \mid 0.8$ & $0.5 \mid 0.7$ \\
\hline$\Omega(0)$ & $1.2 \mid 9.7$ & $1.3 \mid 9.7$ & $1.4 \mid 9.5$ & $1.3 \mid 6.4$ & $3.1 \mid 12.3$ & $0.7 \mid 2.0$ \\
\hline$\Pi(0.5)$ & $7.3 \mid 12.2$ & $7.3 \mid 12.2$ & $7.1 \mid 11.9$ & $6.4 \mid 11.0$ & $4.4 \mid 11.1$ & $2.0 \mid 2.8$ \\
\hline$\eta$ & $0.0 \mid 0.0$ & $0.1 \mid 1.1$ & $0.9 \mid 5.3$ & $1.3 \mid 6.3$ & $0.7 \mid 3.2$ & $0.2 \mid 0.7$ \\
\hline \multicolumn{7}{|c|}{$n=0.3, \tau_{\min }=10^{-20}$} \\
\hline$\tau_{\max }$ & $10^{10}$ & $10^{10}$ & $10^{10}$ & $1.3 \cdot 10^{9}$ & $3.5 \cdot 10^{2}$ & $4.5 \cdot 10^{-3}$ \\
\hline$\gamma$ & $1.0 \mid 2.8$ & $1.0 \mid 2.6$ & $0.7 \mid 1.9$ & $0.3 \mid 0.9$ & $0.2 \mid 0.9$ & $0.1 \mid 0.8$ \\
\hline$\Omega(0)$ & $2.8 \mid 7.0$ & $2.8 \mid 7.0$ & $2.9 \mid 7.0$ & $2.8 \mid 6.9$ & $4.2 \mid 7.2$ & $3.3 \mid 6.1$ \\
\hline$\Pi(0.5)$ & $7.1 \mid 12.1$ & $7.1 \mid 12.1$ & $6.9 \mid 11.4$ & $6.5 \mid 10.9$ & $5.3 \mid 9.9$ & $3.5 \mid 7.5$ \\
\hline$\eta$ & $0.0 \mid 0.0$ & $0.1 \mid 1.3$ & $0.9 \mid 6.2$ & $1.6 \mid 7.5$ & $0.5 \mid 2.2$ & $0.8 \mid 1.9$ \\
\hline
\end{tabular}

